\title{
22ND Annual Meeting Abstracts
}

\section{IMPLEMENTATION OF A PHARMACIST MAN- AGED PARENTERAL NUTRITION SERVICE FOR MEDICAL AND SURGICAL PATIENTS AT A PE- DIATRIC HOSPITAL}

Claire Babcock and Megan Nimke

Children's Hospitals and Clinics of Minnesota, Minneapolis, MN, USA claire.babcock@childrensmn.org

PURPOSE: The purpose of this study is to describe the implementation of a pharmacist managed PN service at an urban, pediatric hospital, and discuss the effects of the service on patient care and outcomes. METHODS: This Institutional Review Board approved study was completed in two phases. Phase one consisted of a retrospective chart review of medical and surgical patients $0-18$ years old who received PN between January 1st, 2011 and October 31st, 2011. This group consisted of only patients who had received provider managed $\mathrm{PN}$, and acted as the historical comparator for pharmacist managed PN. Phase two included prospective implementation and retrospective evaluation of pharmacist managed PN. Data collection for phase two continued until sample size matched phase one. Patients with intensive care status and patients continuing home PN while inpatient were excluded from the study. Each patient chart was reviewed for appropriateness of PN management based on the American Society of Parenteral and Enteral Nutrition's standards for nutritional support in pediatric patients. Main outcome measures included number of days NPO or hypocaloric intake prior to initiation of PN, baseline labs ordered, availability of dietician assessment, frequency of laboratory monitoring while on PN, episodes of hyperglycemia and hypophosphatemia, and length of stay. Implementation of the service included stake holder input, policy development, and competency training for pharmacists. RESULTS: Statistical analysis included a total of 68 patients, with 34 patients identified for both phase one and phase two. Mean number of days NPO or hypocaloric intake was significantly decreased for phase 2 (3.94 days versus 6.12 days, $\mathrm{p}<0.001$ ). Duration of PN and inappropriate PN use did not differ significantly between the groups. Baseline laboratory monitoring was similar for both groups, however, frequency of laboratory monitoring occurred significantly more than recommended with provider managed $\mathrm{PN}(76.5 \%$ versus $29.4 \%, \mathrm{p}<0.001)$. Availability of dietician assessment was significantly increased in phase $2(79.4 \%$ versus $38.2 \%, p=0.001$ ). Episodes of hyperglycemia and hypophosphatemia did not differ significantly between the two groups. Length of stay was approximately four days shorter with pharmacist managed $\mathrm{PN}$, although this difference did not reach statistical significance.
CONCLUSIONS: The implementation of a pharmacist managed PN service resulted in increased interdisciplinary collaboration and a decrease in excessive laboratory monitoring. The results of this study suggest earlier nutritional intervention may lead to a decreased length of stay in the pediatric population, but further study is needed to confirm this finding.

\section{RETROSPECTIVE EVALUATION OF ENOXAPA- RIN DOSING IN INFANTS AND CHILDREN \\ Scott Nelson, ${ }^{1,2}$ Lindsay Butterfield, ${ }^{1}$ \\ and Emily Benefield ${ }^{1}$ \\ ${ }^{1}$ Primary Children's Medical Center, Salt Lake City, UT, USA, ${ }^{2}$ University of Utah College of Pharmacy, Salt Lake City, UT, USA. emilygish@gmail.com}

BACKGROUND: Enoxaparin is recommended by the CHEST guidelines as a therapeutic option when treating thromboembolism in pediatric patients. Current dosing recommendations for infants younger than 2 months-of-age and those older than 2 monthsof-age are $1.5 \mathrm{mg} / \mathrm{kg} /$ dose and $1 \mathrm{mg} / \mathrm{kg} /$ dose subcutaneously every 12 hours respectively. This study evaluates these dosing recommendations and their ability to achieve therapeutic anti-factor Xa levels. METHODS: This retrospective review assessed all children younger than 18 years-of-age receiving full anticoagulation therapy with enoxaparin from January 1, 2010 to March 1, 2012. Subjects were excluded if their calculated glomerular filtration rate was $<30 \mathrm{~mL} / \mathrm{min} / 1.73 \mathrm{~m} 2$ or if no anti-factor Xa monitoring was performed. The primary outcome was the average enoxaparin dose required to reach therapeutic anti-factor Xa concentration of 0.5-1 units $/ \mathrm{mL}$. Secondary outcomes assessed include the percentage of patients achieving a therapeutic antifactor Xa level on the first laboratory measurement, the number of dose changes, and major bleeding complications. Data were analyzed descriptively. RESULTS: A total of 293 patients receiving enoxaparin were identified with 154 patients who met inclusion criteria. For patients younger than 2 months-of-age, the average therapeutic dose required was $2.00 \mathrm{mg} /$ $\mathrm{kg} /$ dose after an average of 1.67 dose changes. For patients over 2 months-of-age, the average therapeutic dose required was $1.16 \mathrm{mg} / \mathrm{kg} /$ dose after an average of 1.03 dose changes. It was noted that further breakdown could help elucidate the dosing differences seen based on age. The average therapeutic dose was 2.00 $\mathrm{mg} / \mathrm{kg} /$ dose for patients less than 3 months-of-age, $1.51 \mathrm{mg} / \mathrm{kg} /$ dose for patients 3 months to 1 year-ofage, $1.21 \mathrm{mg} / \mathrm{kg} /$ dose for patients 1 to 5 years-of-age, and $1.02 \mathrm{mg} / \mathrm{kg} /$ dose for children over 5 years-of-age. Infants 3 months to 1 year-of-age were most likely to 
achieve a therapeutic anti-factor Xa activity at the first measurement compared to other age groups (48.3\%). Patients younger than 3 months-of-age were most likely to have a low anti-factor Xa activity (61.3\%) and children 1 to 5 years-of-age were most likely to have a high anti-factor Xa activity $(10.5 \%)$ on the first measurement. There were 7 instances of major bleeding, and only one patient had a supratherapeutic anti-factor Xa level while receiving enoxaparin. Additionally, there were 5 documented episodes of minor bleeding in the treatment population. CONCLUSIONS: Based on the CHEST guidelines' recommended dosing, therapeutic anti-factor $\mathrm{Xa}$ activity were achieved in less than half of all pediatric patients assessed. The majority of anti-factor Xa measurements were low initially and required at least one dose adjustment to reach therapeutic levels. Based on these findings, higher initial dosing recommendations may be needed in order to achieve therapeutic antifactor Xa activity in pediatric populations. Further research is warranted.

\section{A RETROSPECTIVE REVIEW OF ENOXAPARIN DOSING IN THE TREATMENT OF VENOUS THROMBOEMBOLISM IN THE PEDIATRIC POPULATION}

Emily Wells, ${ }^{1}$ Kim Benner, ${ }^{1,2}$ Mary Worthington,, ${ }^{1,2}$

Emily Waite, ${ }^{2}$ and Christy Bemrich-Stolz ${ }^{3}$

${ }^{1}$ Samford University McWhorter School of Pharmacy, Homewood, AL, USA, ${ }^{2}$ Children's of Alabama Pharmacy Department, Birmingham, AL, USA, ${ }^{3}$ University of Alabama at Birmingham,Birmingham, AL, USA.kwbenner@ samford.edu

INTRODUCTION: Venous thromboembolism (VTE) is an uncommon event among the pediatric population with an increase in recognition over the past years, allowing for advancements in current treatment recommendations. There have been challenges seen in relation to the dosing of enoxaparin and the ability to achieve therapeutic anti factor-Xa levels, subsequently requiring multiple dosage adjustments. The purpose of this study was to determine if the current initial dose of enoxaparin, recommended by the American College of Chest Physicians(ACCP) for the treatment of VTE in children, achieved therapeutic anti factor-Xa levels among patients ages 0-18 years. METHODS: After obtaining institutional review board approval, a retrospective review was conducted. All pediatric patients, ages $0-18$ years, treated for VTE with enoxaparin as inpatients at Children's of Alabama between January 2010 and June 2012 were included. Demographic patient data as well as data pertaining to the dosing and monitoring of enoxaparin was collected and analyzed by review of electronic medical records. The primary endpoint was to determine the final dose of enoxaparin needed to achieve a therapeutic anti factor-Xa level, with a secondary endpoint being the amount of dosage adjustments required to reach therapeutic level. RESULTS: Using a one-way ANOVA with a 95\% confidence interval, a significant statistical difference was seen between the mean dose of enoxaparin required to achieve therapeutic anti factor-Xa levels between children less than one year old and those greater than one year old. Among the 49 patients who received enoxaparin for the treatment of thrombosis, 39\% required at least one dose adjustment due to anti factor-Xa levels outside of therapeutic range, with $53 \%$ of those patients requiring more than one dose adjustment. There was no statistically significant difference seen among those patients started at an appropriate dose of enoxaparin and those initiated at either a higher or lower dose as to whether they would require a dose adjustment. CONCLUSION: This study concluded that the current ACCP recommended initial enoxaparin dose for the treatment of VTE in children may not be consistently effective at achieving therapeutic anti factor-Xa levels in children less than one year old. However, additional studies are needed to determine if the dose should be increased from the current guidelines in order to achieve therapeutic anticoagulation in a timely manner, as well as reduce the amount of dosage adjustments required.

\section{COMPARISON OF anti-Xa \& aPTT HEPARIN AS- SAY MONITORING FOR PEDIATRIC PATIENTS FOR VTE TREATMENT}

Laura $\mathrm{Bio}^{1,2}$ and Robert McNeil ${ }^{1}$

${ }^{1}$ Philadelphia College of Pharmacy, Philadelphia, PA, USA, ${ }^{2}$ Children's Regional Hospital at Cooper University Hospital, Camden, NJ, USA. l.bio@usp.edu

INTRODUCTION: The objective of this study is to compare anti-Xa and activated partial thromboplastin time (aPTT) heparin assay to determine which method achieves therapeutic anticoagulation more rapidly in pediatric patients receiving unfractionated heparin (UFH) infusion. Currently there is a need to establish an optimal method to monitor UFH in pediatric patients. METHODS: Retrospective, observational cohort study of pediatric patients 1 month to 18 years of age who received UFH continuous infusion between 12/01/2008 and 3/31/2012. Patients were excluded if they had a protein $\mathrm{C}$ or $\mathrm{S}$ deficiency, or a hematologic or thrombotic disorder. Patients were separated into two groups: monitored with anti-Xa heparin assay (HA) and monitored with aPTT HA. Therapeutic anticoagulation was defined as 0.35-0.7 anti-Xa units/mL for the anti-Xa group and 67-101 seconds for aPTT group. The primary outcome was a comparison of time to therapeutic anticoagulation between the two groups. Secondary outcomes included a comparison of time within therapeutic anticoagulation, duration of UFH infusion, number of infusion rate adjustments. Descriptive analysis of 
the data was preformed, and the primary outcome was statistically analyzed using the Fisher's exact test. RESULTS: Nine patients received continuous UFH between the time period studied: five patients were included in the anti-Xa HA group and four patients in the aPTT group. One patient in each group had a pulmonary embolism as the indication for continuous UFH, and the remaining 4 and 3 patients in the anti-Xa and aPTT group, respectively had deep vein thrombosis (DVT). Two patients $(40 \%)$ in the anti-Xa group reached therapeutic anticoagulation in the first 24 hours compared to zero patients in the aPTT group $(p=0.44)$. All five anti-Xa HA patients achieved therapeutic anticoagulation within 48 hours and three patients (75\%) in the aPTT group achieved therapeutic anticoagulation within that time $(\mathrm{p}=1)$. All four aPTT patients reached therapeutic anticoagulation by 72 hours after initiation of UFH. CONCLUSION: The anti-Xa HA group trended to achieve therapeutic anticoagulation in less time compared to the aPTT group, however further studies should be conducted to better evaluate this outcome.

\section{A RETROSPECTIVE REVIEW: FREQUENCY OF ZIDOVUDINE-INDUCED HEMATOLOGIC AB- NORMALITIES}

Andrea Budde, ${ }^{1,2}$ Miranda Nelson, ${ }^{1,2}$ and Ericka Hayes $^{2,3}$

${ }^{1}$ Southern Illinois University Edwardsville School of Pharmacy, Edwardsville, IL, USA, ${ }^{2}$ St. Louis Children's Hospital, St. Louis, MO, USA, ${ }^{3}$ Department of Pediatrics, Washington University School of Medicine, St. Louis, MO, USA. andrea.budde@bjc.org

INTRODUCTION: Zidovudine has been recommended for prevention of maternal-fetal vertical human immunodeficiency virus (HIV) transmission since the Pediatric AIDS Clinical Trials Group protocol 076 determined that zidovudine administration to HIVinfected mothers during pregnancy and labor followed by 6-weeks of prophylactic treatment to exposed infants reduced vertical transmission risk by $70 \%$. While this treatment regimen has been proven to effectively reduce transmission, adverse events have been associated with this drug's use including neutropenia, anemia and thrombocytopenia. It is hypothesized that the incidence of adverse events is grossly underestimated; neonatal data has previously shown neutropenia and anemia to occur at rates of $21 \%$ and $22 \%$, respectively. The purpose of this study was to determine the actual incidence of these hematologic alterations. METHODS: Retrospective review of patient charts was completed for all HIV-exposed neonates admitted to St. Louis Children's Hospital from January 2008 to December 2011. Exclusion criteria included insufficient laboratory data, inappropriate dosing regimens and/or presence of blood dyscrasias prior to zidovudine administration. Laboratory and de- mographic information were evaluated for included neonates and mothers. Reviewed laboratory results were from birth, 6 weeks and 1 year of life. A severity/grading scale was created using the Division of Acquired Immunodeficiency Syndrome/National Institute of Allergy and Infectious Diseases grading system of adverse events. Data related to hematologic alterations was evaluated for incidence and severity. RESULTS: Data was collected for 98 neonates. Labs for the 84 patients who met inclusion criteria were evaluated individually to determine eligibility. Sixty patients were included in the neutropenia evaluation; $15(25 \%), 5(8 \%), 4(7 \%)$ and $1(2 \%)$ experienced grade 1-4 neutropenia, respectively. Seventeen (68\%) cases resolved and $8(32 \%)$ did not. Seventy-nine patients were included in the thrombocytopenia evaluation; 3 $(4 \%)$ and $2(2 \%)$ patients experienced grade 1 and grade 2 thrombocytopenia, respectively. All cases resolved. Seventy-six patients were included in the anemia evaluation; 21 (28\%), 24 (31\%), 6 (8\%) and $3(4 \%)$ experienced grade 1-4 anemia, respectively. Forty-four $(81 \%)$ cases resolved and 10 (19\%) did not. Resolution of neutropenia and anemia could not be confirmed for all patients due to lack of laboratory results. CONCLUSION: Results indicate that neutropenia, anemia, and thrombocytopenia occur at higher rates than previously reported. Neutropenia and anemia occurred at two and greater than three times the previously reported incidences, respectively. While the incidence of thrombocytopenia is not currently reported in Micromedex, when the results of this study are compared to the results of similar studies, the incidence of thrombocytopenia in this study is more prevalent. Limitations of this study (i.e., the level of maternal hemoglobin and iron stores were not accounted for and the inability to control for physiologic nadir of infants and gestational age) may have played a significant role in the elevated incidence of parameters.

\section{SAFETY OF A TWO-TIERED MONITORING REQUIREMENT FOR PEDIATRIC PROCEDURE SEDATION}

Jared Cash and Sheldon Furst

Primary Children's Medical Center, Salt Lake City, UT, USA jared.cash@imail.org

BACKGROUND: In an effort to improve the safety of pediatric procedural sedation, regulatory bodies in consultation with various professional organizations issued guidelines for appropriate sedation practices. These in-depth requirements are specifically designed for moderate and deep procedural sedation. Requirements for a lower level than moderate sedation are not specifically addressed. Many institutions in order to be compliant ask all procedure sedations to follow the same requirements, thus a single tier approach. Primary Children's Medical Center proposed that there was a subset of medications and doses that could be 
safely administered for procedures using less stringent monitoring requirements, thus freeing up staff and increasing efficiency. This lower requirement tier is called minimal sedation and is different in that only certain low-risk medications within a specific dose range are allowed. Only one dose of medication is permitted and no other pain medication or sedatives can be given within 60 minutes. The prescriber does not need to be specifically credentialed for sedation. Adequate staffing and equipment, including bag and mask, must be immediately available. If a degree of sedation is deeper than " $\mathrm{A}$ " on the NAMDU scale or the patient has an increased oxygen requirement to maintain $\mathrm{SPO} 2$ greater than $90 \%$ then the full sedation protocol must be enacted. The objective of this study is to evaluate the safety of this minimal sedation requirement at our institution since its inception in 2010. METHODS: The procedural sedation records from August 2010 to January 2013 for minimal sedation were analyzed for medications used, procedures performed, noted complications, location, and need to progress to full sedation protocol. 1413 records were identified. 136 were excluded as inappropriately categorized due to the medications used which left 1277 sedation records for analysis. RESULTS: Midazolam was used in $95 \%$ of the procedures, $70 \%$ nasal route, $15 \%$ oral and $15 \%$ intravenous. The Emergency Department was the most frequent procedural unit at $80 \%$. Laceration repair was the most common procedure at $57 \%$ followed by imaging at $9 \%$. $6 \%$ (75) of the procedures required a switch to full sedation protocol. Excluding these, 88\% (1245) were optimally sedated. $<1 \%$ (3) cases were over sedated and $12 \%$ (165) were under sedated. There were 5 cases of cardiac adverse events (low heart rate or hypotension) with 2 requiring a fluid bolus, 3 cases of nausea/vomiting, 1 case of paradoxical agitation, 2 cases of oxygen saturation changes (stayed above $90 \%$ ) and 1 patient with apnea greater 20 seconds not requiring intervention. The average percentage of minimal sedations as a total of all sedations was 12\% for the year 2012 . CONCLUSION: The Emergency Department is the predominant user of the minimal sedation procedure with intranasal midazolam as the predominant medication. A two tiered approach to pediatric procedural sedation requirements appears to be a safe alternative to a single tiered approach in a pediatric medical center.

ETHANOL LOCK THERAPY FOR THE TREATMENT OF CENTRAL LINE-ASSOCIATED BLOODSTREAM INFECTIONS IN HOSPITALIZED CHILDREN RECEIVING PARENTERAL NUTRITION

Rebecca Chhim, Catherine Crill, and

Michael Christensen

The University of Tennessee Health Science Center, Memphis, TN, USA, Le Bonheur Children's Hospital, Memphis, TN, USA rchhim@uthsc.edu
INTRODUCTION: Central line-associated bloodstream infection (CLABSI) remains a serious complication associated with central venous catheters (CVC) in hospitalized patients receiving parenteral nutrition (PN). Ethanol lock therapy (ELT) has emerged as an effective alternative to antibiotic lock therapy (ALT) for the prevention of CLABSI in adult and pediatric patients with a history of recurrent CLABSI. Current treatment guidelines recommend the use of ALT for catheter salvage in select patients, but due to limited data, ELT is not included in these recommendations. At our institution, ELT has been used since 2007 for the prevention and treatment of CLABSI in select pediatric patients receiving long term PN therapy. The objective of this study was to evaluate ELT for CLABSI treatment in these patients. METHODS: This retrospective review evaluated ELT for CLABSI in hospitalized patients receiving chronic PN. Subjects included home PN patients or hospitalized patients on long-term PN with CLABSI. All patients received ELT daily during PN off cycle (instilled into the CVC and allowed to dwell for at least 2 hours) in addition to systemic antimicrobial CLABSI therapy. Data collection included patient age and weight, blood culture results (peripheral and central), duration of ELT, catheter removal due to failure to eradicate the infection, and catheter salvage at 30 days. RESULTS: From November 2009 through January 2013, 13 patients; ages 0.5-23 years, weighing 5.4-68 $\mathrm{kg}$; received a total of 17 courses of ELT for treatment of CLABSI. Ten patients received 1 course each, 2 patients received 2 courses each, and 1 patient received 3 courses of ELT. In patients who received more than one ELT course, the courses were separated by at least 30 days. Successful eradication of the infection occurred with 13 courses $(76 \%)$ while successful catheter salvage at 30 days was achieved in 11 courses (65\%). The CVC was removed for failure to eradicate the infection in 4 courses (1 each - Candida parapsilosis, Enterococcus faecalis, methicillin-resistant Staphylococcus aureus, and Enterococcus faecium). The CVC was removed in 2 additional courses due line malfunction within 30 days (no evidence of continued CLABSI). CONCLUSIONS: ELT has the potential to be used as an alternative to ALT in the treatment of CLABSI. Potential advantages of ELT include effectiveness against a wide range of microorganisms, no evidence of resistance after exposure, and the ability to penetrate and sterilize biofilms in the CVC. Randomized studies are needed to compare the effectiveness of ELT and antibiotic lock therapy in patients with CLABSI.

\section{THE IMPACT OF A PHARMACIST-MANAGED RSV PREVENTION CLINIC ON PALIVIZUMAB COMPLIANCE AND RSV ASSOCIATED HOSPI- TALIZATION: A 3 YEAR EXPERIENCE}

Jennifer Chow, ${ }^{1}$ Michael Chicella, ${ }^{1,2}$ and James Dice ${ }^{1}$ 
${ }^{1}$ Children's Hospital of The King's Daughters, Norfolk, VA, USA, ${ }^{2}$ Eastern Virginia Medical School, Norfolk, VA, USA jwoo79@hotmail.com

PURPOSE: Respiratory syncytial virus (RSV) is the most common cause of bronchiolitis and pneumonia in infants and is the leading cause of hospitalizations in infants less than one year of age in the United States. Palivizumab is an RSVspecific monoclonal antibody that is administered once monthly during RSV season to prevent RSV infection. Non-compliance with the dosing regimen is the factor commonly associated with drug failure. The purpose of this study was to determine the impact of a pharmacist-managed RSV Prevention Clinic on palivizumab compliance and RSVassociated hospitalization over the last three years. METHODS: During the 2009-2010 RSV season, the pharmacist-managed RSV Prevention Clinic was established. During the clinic visit, the pharmacist was responsible for providing education about RSV and administering palivizumab to the patient. In order to determine the impact of this clinic we compared data from the 2008-2009 RSV season (group 1) which was prior to the establishment of the clinic to data from the 2009-2010 RSV season (group 2), 2010-2011 season (group 3) and 2011-2012 season (group 4). Groups two, three, and four were after the clinic was established. Data compared included demographics, number of shots administered, RSV testing, and RSVrelated hospitalizations ( $>24$ hours in hospital). Compliance was determined as the number of patients who received all eligible doses in consecutive months. RESULTS: Eighty-six infants were eligible for palivizumab in group 1 and 75, 57, and 54 infants were eligible in groups 2, 3, and 4 respectively. Sixty-seven infants $(78 \%)$ in group 1 received at least one dose of palivizumab, whereas 71 infants $(95 \%)$ in group 2, 53 infants (93\%) in group 3, and 48 infants $(89 \%)$ in group 4 received one dose. Nineteen infants $(28 \%)$ in group 1 received all doses of palivizumab as compared to 63 infants (89\%), 50 infants $(94 \%)$, and 45 infants (94\%) in groups 2, 3, and 4 respectively $(\mathrm{p}<0.0001)$. With regard to compliance, only 18 patients $(27 \%)$ in group 1 received all eligible doses in consecutive months as compared to 60 patients $(85 \%)$ in group 2,47 patients $(89 \%)$ in group 3 , and 43 patients $(90 \%)$ in group $4(\mathrm{p}<0.0001)$. In group 1, eight patients developed RSV and 5 of those required hospitalization. In group 2, three patients developed RSV but only one required hospitalization. No patients in group 3 developed RSV and one patient in group 4 developed RSV, but did not require hospitalization $(\mathrm{p}<0.04)$. CONCLUSIONS: The pharmacist-managed RSV Prevention Clinic statistically improved compliance with palivizumab and decreased RSV infections and hospitalizations by over $50 \%$.
POPULATION PHARMACOKINETICS (PK) OF PIPERACILLIN/TAZOBACTAM (TZP) IN CRITICALLY ILL CHILDREN

Jeffrey Cies, ${ }^{1}$ Venkat Shankar, ${ }^{3}$ Christine Schlichting, ${ }^{1,2}$ and Joseph Kuti ${ }^{4}$

${ }^{1}$ St. Christopher's Hospital for Children, Philadelphia, PA, USA, ${ }^{2}$ Drexel University College of Medicine, Philadelphia, PA, USA, ${ }^{3}$ Children's National Medical Center, Washington, DC, USA, ${ }^{4}$ Center for Anti-Infective Research and Development, Hartford Hospital, Hartford, CT, USA jeffrey. cies@gmail.com

INTRODUCTION: TZP is a frequently prescribed antibiotic in pediatric intensive care units (PICU), but PK data to justify the optimal TZP dosing regimen are sparse in critically ill children.METHODS: Blood samples (2-4 per child) were collected from 13 children ages 9 months -6 years admitted to the PICU who were receiving standard TZP dosing regimens to treat infections. Piperacillin (PIP) concentrations were measured by a bioassay and the population PK of the TZP component was conducted using non-parametric adaptive grid (NPAG) with adaptive $\gamma$. Multiple models were tested to determine the best fit of the data. A 5000 patient Monte Carlo simulation (MCS) was performed to determine the probability of target attainment (PTA) for the following TZP dosing regimens $(100 \mathrm{mg} / \mathrm{kg}$ q6h as 0.5 and 4 hour infusions) in a population of 1-6 year old male children. CDC weight for age charts were used as weight distributions. The percent of the dosing interval the free drug is above the minimum inhibitory concentration (MIC) (fT $>\mathrm{MIC})$ was calculated over a range of MICs from $0.03-128 \mathrm{mg} / \mathrm{L}$. The bactericidal target attainment was defined as $\geq 50 \%$ fT $>$ MIC for TZP. PTA $\geq 90 \%$ at each MIC was defined as optimal. RESULTS: A 2 compartment model fit PIP concentration data the best. Mean (SD) population estimates for clearance (CL), volume of the central compartment (Vc), and intercompartment transfer constants were 4.44(2.82) $\mathrm{L} / \mathrm{hr}, 3.71(1.94) \mathrm{L}, 3.13(3.73) \mathrm{h}-1$, and 7.23(6.72) hr-1, respectively. This resulted in a mean (SD) elimination half-life of 1.4(0.79) h. Weight adjusted estimates for CL, $\mathrm{Vc}$ and total volume of distribution were $0.29 \mathrm{~L} / \mathrm{hr} /$ $\mathrm{kg}, 0.27 \mathrm{~L} / \mathrm{kg}$ and $0.51 \mathrm{~L} / \mathrm{kg}$, respectively. The bias and precision for the individual predicted versus observed concentrations were -0.14 and $1.69 \mathrm{mg} / \mathrm{L}$, respectively. PTAs for the TZP dosing regimens are as follows:

\begin{tabular}{|c|c|c|}
\hline \multirow{2}{*}{ MIC (mg/L) } & \multicolumn{2}{|c|}{ PTA (\%) } \\
\cline { 2 - 3 } & $\begin{array}{c}100 \mathrm{mg} / \mathrm{kg} \mathrm{q} 6 \mathrm{hr} \\
\text { (0.5 hr infusion) }\end{array}$ & $\begin{array}{c}100 \mathrm{mg} / \mathrm{kg} \mathrm{q} 6 \mathrm{hr} \\
\text { (4 hr infusion) }\end{array}$ \\
\hline 2 & 100 & 100 \\
\hline 4 & 99.7 & 100 \\
\hline 8 & 98.2 & 100 \\
\hline 16 & 88.9 & 99.9 \\
\hline 32 & 37 & 93.1 \\
\hline 64 & 2.6 & 47 \\
\hline 128 & 0 & 4.8 \\
\hline
\end{tabular}


CONCLUSION: These are the first PK data of TZP (PIP component) in critically ill pediatric patients (1-6 years of age). Based on these data, $100 \mathrm{mg} / \mathrm{kg} \mathrm{q} 6 \mathrm{hr}$ doses administered as 0.5 hour infusions will provide optimal PTA up to TZP MICs of $8 \mathrm{mg} / \mathrm{L}$, where as prolonged 4 hour infusions will increase optimal PTA.

INTRAVENOUS ENOXAPARIN USE IN PEDIATRIC INTENSIVE CARE UNIT PATIENTS

Jeffrey Cies,,$^{1,2}$ Laura Santos,,$^{3,4}$ and Arun Chopra ${ }^{1,2}$

${ }^{1}$ St. Christopher's Hospital for Children, Philadelphia, PA, USA, ${ }^{2}$ Drexel University College of Medicine, Philadelphia, PA, USA, ${ }^{3}$ NYU Langone Medical Center, New York, NY, USA, ${ }^{4} N Y U$ School of Medicine, New York, NY, USA jeffrey.cies@gmail.com

INTRODUCTION: Titration and administration of subcutaneous (SC) enoxaparin (Ep) is often difficult in certain distinct populations, such as premature infants and critically ill children with severe edema. Difficulty achieving adequate anticoagulation in this population has led to increased use of intravenous (IV) Ep. Sparse pharmacodynamic data are available for determining the appropriate dose, infusion, duration, and monitoring (by anti-Factor Xa levels) of IV Ep. The objective of this study is to report our experience with the use of IV Ep in neonatal and pediatric patients in the intensive care unit. METHODS: We performed a retrospective chart review from 1/1/09-6/30/12 of patients who received IV Ep and had $\geq 1$ anti-10a level reported. Ep doses were infused over 30 minutes and anti-10a levels were drawn 4 hours after the start of the infusion. RESULTS: 15 patients were included. 14 of 15 patients received IV Ep for treatment and 1 for prophylaxis. Patient ages ranged from 21d-16yrs with a median weight of $5 \mathrm{~kg}$. The median duration of IV therapy was 11 days (range 1-120). The mean initial IV dose was $1.14 \pm 0.38$ $\mathrm{mg} / \mathrm{kg} /$ dose $\mathrm{q} 12 \mathrm{hr}$ and the mean therapeutic IV dose was $1.31 \pm 0.52 \mathrm{mg} / \mathrm{kg} /$ dose q $12 \mathrm{hr}$. There were no adverse events reported related to bleeding, thrombosis, or hypersensitivity in the patients evaluated. CONCLUSION: The pharmacodynamics of a $30 \mathrm{~min}-$ ute IV Ep infusion was found to produce therapeutic 4 hour anti-10a levels similar to SC doses. Although this was a small study, there were no adverse events, suggesting the safety profile may be similar to SC dosing with less pain. These findings suggest that IV Ep may be a viable option for anticoagulating critically ill children and its use warrants further study.

\section{PROCALCITONIN (PCT) USE IN A PEDIATRIC} INTENSIVE CARE UNIT

Jeffrey Cies, ${ }^{1,2}$ Mindy Dickerman, ${ }^{1,2}$ and Arun Chopra ${ }^{1,2}$ ${ }^{1}$ St. Christopher's Hospital for Children, Philadelphia, PA, USA, ${ }^{2}$ Drexel University College of Medicine, Philadelphia, PA, USA jeffrey.cies@gmail.com
BACKGROUND: Procalcitonin (PCT) levels are of interest as a biomarker in intensive care unit (ICU) patients. PCT levels are elevated in patients with bacterial pneumonia and septic shock. Current data suggest that serum PCT levels are not elevated in patients with viral respiratory tract infection unless there is a secondary bacterial infection. Serum PCT levels have the potential to inform the clinical decision to empirically treat ICU patients with antimicrobial therapy. In our institution PCT was adopted to aid in guiding antimicrobial therapy. METHODS: We conducted a retrospective chart review of all patients with at least one PCT level from 12/1/2011 thru 4/30/12. Medical records of patients were reviewed to determine the diagnosis of infection using clinical, biological, microbiological and radiological data. The diagnostic utility of the PCT test in patients with bacterial infections was compared to the utility in patients with non-bacterial infections. RESULTS: 154 patients met the inclusion criteria and accounted for 334 PCT samples. There was a positive correlation between an $\mathrm{PCT} \geq 1 \mathrm{ng} / \mathrm{mL}$ and a positive bacterial culture, Pearson's correlation coefficient $(\mathrm{PCC})=0.279, \mathrm{p}=0.01$. There was a positive correlation between a PCT $\geq 1 \mathrm{ng} / \mathrm{mL}$ and receiving a treatment course of antimicrobials, $\mathrm{PCC}=0.48$, $\mathrm{p}=0.01$. Logistic regression analysis demonstrated that a PCT $\geq 1 \mathrm{ng} / \mathrm{mL}$ predicted having a positive bacterial culture, OR=2.6 (95\% CI 1.3-4.9). A PCT $\geq 1$ $\mathrm{ng} / \mathrm{mL}$ had a positive predictive value (PPV) of $42 \%$, a negative predictive value (NPV) of $85 \%$, a sensitivity of $62 \%$ and a specificity of $71 \%$. Using logistic regression, a PCT $<1 \mathrm{ng} / \mathrm{mL}$ predicted the absence of a bacterial infection, OR=4.1 (95\% CI 2.1-7.9). CONCLUSION: Within a pediatric ICU, a PCT cutoff value of $1 \mathrm{ng} / \mathrm{mL}$ demonstrated a NPV of $85 \%$ and a PPV of $42 \%$. These data suggest PCT can assist in identifying patients with bacterial infections and may help guide when to use and continue empiric antimicrobials. In addition, one needs to consider other etiologies for clinical deterioration when a patient presents with low PCT and worsening clinical course. The use of PCT in a pediatric ICU needs to be validated in a prospective manner.

\section{A RETROSPECTIVE REVIEW OF AMINOPHYL- LINE FOR ACUTE KIDNEY INJURY IN THE NEONATE \\ Colleen Cloyd, Shabnam Gaskari, and Adam Frymoyer \\ Lucile Packard Children's Hospital at Stanford, Palo Alto, CA, USA ccloyd@lpch.org}

BACKGROUND: Small retrospective studies suggest that aminophylline may help improve renal function in pediatric patients with acute kidney injury (AKI). This potential therapeutic strategy in neonates with AKI has not been fully explored. 
Currently, the dose of aminophylline for AKI in neonates is based on previous reports in older pediatric populations. However, neonates display profound differences in drug kinetics due to size and maturational considerations; therefore, the current dosing regimen may not be appropriate for the neonate. OBJECTIVES: To evaluate the current aminophylline dosing regimen used in neonates with AKI in terms of drug concentration achievement and impact on kidney function. METHODS: A retrospective chart review of neonates with AKI treated with aminophylline in the neonatal intensive care unit at Lucile Packard Children's Hospital (LPCH) was performed. Patient demographics and medication history including aminophylline dose history, and theophylline drug concentrations were collected. Renal function was assessed before and after the start of aminophylline using urine output (UOP) and serum creatinine (SCr). RESULTS: Twenty-nine neonates with AKI received aminophylline over a 3.5 year period. The most common dosing regimen used was a loading dose of 5 $\mathrm{mg} / \mathrm{kg} \pm 10 \%$ followed by $1.8 \mathrm{mg} / \mathrm{kg} \pm 10 \%$ every 6 hours $(n=19)$. The mean duration of therapy was 6.3 days (range: 1-53). Initial theophylline levels for only $9(31 \%)$ patients were in the LPCH goal range for renal perfusion (5-7 mg/L). Thirteen (45\%) patients had a theophylline concentration $>7 \mathrm{mg} / \mathrm{L}$ at some point during therapy. Neonates that achieved consistent therapeutic levels received an average regimen of $1.5 \mathrm{mg} / \mathrm{kg} /$ dose every 6 hours. Preliminary results demonstrated that aminophylline use was associated with increased mean UOP with a subsequent decrease in mean SCr after 5 days of therapy (115.9 $\mathrm{mL}$ vs. $306.7 \mathrm{~mL}, \mathrm{p}=0.0001$ and $1.1 \mathrm{mg} / \mathrm{dL}$ vs. 0.8 $\mathrm{mg} / \mathrm{dL}, \mathrm{p}=0.009$, respectively). There were no serious adverse effects attributed to the use of aminophylline. CONCLUSIONS: Aminophylline was associated with improvement in UOP and a decrease in SCr when used in neonates with AKI. However, the current aminophylline dosing regimen frequently resulted in drug concentrations outside of the therapeutic target levels. Therefore, a customized dosing strategy for this patient population will be necessary. Further pharmacokinetic and prospective clinical studies are warranted to help optimize this potential therapeutic strategy in neonates with AKI.

INITIATION AND MAINTENANCE OF WIRELESS SMART PUMP TECHNOLOGY IN A NEW CHILDREN'S HOSPITAL

Katie Conrad and Angela Rollins

Nemours Children's Hospital, Orlando, FL, USA kconrad@ nemours.org

BACKGROUND: Nemours Children's Hospital is a 95-bed free standing children's hospital in Orlando, Florida. The hospital opened to patients in October
2012 and went live with the Medfusion 4000 syringe pumps as the primary method of medication delivery for all medications delivered in syringes. The pumps have the advantage of Pharmguard infusion software as well as wireless technology. An initial drug library was developed with key stakeholders, including pharmacy, nursing, and physician leadership. Pump education was completed with all nurses during general orientation in July 2012. OBJECTIVE: Ensure greater than $90 \%$ compliance with use of Pharmguard infusion software. Action Steps: After three monthly library updates based on nursing and physician feedback, compliance with usage of Pharmguard limits was below target. Pharmacy met with nursing leadership to discuss action steps to improve compliance with the safety software. It was decided that additional feedback from bedside nurses was needed to understand how the drug library did not match their clinical practice. These discussions identified education as a primary component of noncompliance. Pharmacy participated in the mandatory nursing skills fair and was able to educate and have dialogue with all nurses using the pumps. This dialogue led to significant insight about library design and function, including the flush feature, wild card entries, alarms, and understanding of hard and soft limits. It also allowed for reemphasis of expectations of nursing actions in the event the library is not working as intended. Based on this discussion and feedback, a revised drug library was released on March 15, 2013. RESULTS: Drug library compliance was assessed at least monthly before the revised library implementation with average drug library compliance ranging from $53.8 \%$ to $72 \%$. It will be assessed weekly after the new library implementation. Additionally, trends in compliance by profile, time of day, and hard limits and reprograms with specific medications will be assessed. CONCLUSIONS: Our hypothesis is that compliance will increase after the new library is released and reeducation completed. Additionally, it was identified that drug library compliance rounds done randomly by educators and charge nurses would continue dialogue about drug library challenges and ensure that it is being used appropriately.

\section{ACID SUPPRESSING AGENTS AND RISK FOR CLOSTRIDIUM DIFFICILE INFECTION IN PEDI- ATRIC PATIENTS}

Katelyn E. Jensen, ${ }^{2}$ Kristen R. Nichols, ${ }^{1,3}$

Chad A. Knoderer, ${ }^{3}$ and Ashley S. Crumby ${ }^{1,2}$

${ }^{1}$ Riley Hospital for Children, Indianapolis, Indiana, USA, ${ }^{2}$ Purdue University, West Lafayette, Indiana, USA, ${ }^{3} B u t-$ ler University, Indianapolis, Indiana, USA acrumby@ iuhealth.org

INTRODUCTION: Acid suppressing agents, such as histamine 2 receptor antagonists (H2RA) and proton pump inhibitors (PPI) have been as- 
sociated with increased Clostridium difficile infection (CDI) in adults. The objective of this study was to evaluate the association of acid suppressing therapy and the development of Clostridium difficile infection in the pediatric population. METHODS: This was a retrospective case-control study. Children aged 1 through 17 years with a positive $C$. difficile polymerase chain reaction (PCR) obtained between June 1, 2008 and June 1, 2012 were randomly matched 1:1 to a control selected from patients with negative PCR during the same period. Clostridium difficile infection was defined by positive PCR. Demographic data and use of H2RA or PPI prior to PCR were extracted from patient medical records. Presence of possible CDI risk factors including hospitalization within the past 3 months, duration of hospitalization prior to positive/negative PCR, co-morbid conditions, prior antibiotic use, oral steroids, immunosuppressants, prokinetic agents, and chemotherapeutic agents was also recorded. RESULTS: Four hundred and fifty-eight (229 PCR positive and 229 PCR negative) children were included. Children with CDI were younger than children without CDI (median [IQR] years 6 [2-11] vs. 8 [3-11], $\mathrm{p}=0.012$ ). No difference was observed in acid suppressive therapy prior to $\mathrm{PCR}$ in CDI positive vs. negative patients $(57.2 \%$ vs. $52.8 \%, \mathrm{p}=0.348)$. Of patients with acid suppressing therapy prior to obtaining a PCR, more CDI positive patients received acid suppressive therapy at home. (76.3\% vs. $60.3 \%, p=0.006)$. In this subset, no difference was observed in PPI utilization $(45 \%$ vs. $46.3 \%, \mathrm{p}=0.843)$ between CDI positive and negative patients, but H2RA utilization was greater in the CDI positive patients $(32.8 \%$ vs. $14.9 \%$, $\mathrm{p}=0.001)$. Risk factors for CDI were not different between the two groups. Logistic regression analysis demonstrated that H2RA therapy at home (OR: 2.9; 95\% CI: 1.47-5.71) was an independent CDI predictor. CONCLUSION: In this pediatric population, children receiving home acid suppressive therapy with $\mathrm{H} 2 \mathrm{RAs}$ have nearly 3 times greater risk for CDI than children not receiving H2RA therapy. These results suggest the need for continued monitoring and study of H2RA use in the pediatric population.

\section{INITIAL VANCOMYCIN DOSING ASSESSMENT IN NEONATES THROUGH CLINICAL OBSERVA- TION OF LEVELS}

Deonne Dersch-Mills, ${ }^{1}$ Tanner Bengry, ${ }^{1}$ Albert Akierman, ${ }^{1,2}$ Belal Alshaikh, ${ }^{2}$ and Kamran Yusuf ${ }^{1,2}$ ${ }^{1}$ Alberta Health Services, Calgary. Alberta, Canada, ${ }^{2}$ Universoty of Calgary, Calgary, Alberta, Canada deonne. dersch-mills@albertahealthservices.ca

INTRODUCTION: Coagulase negative staphylococcus (CoNS), is the leading cause of late onset sepsis in the neonates, thus there is a high need to empirically dose vancomycin appropriately in neonates.
The Infectious Disease Society of America's (IDSA) suggests trough levels of vancomycin of $10-20 \mathrm{mg} / \mathrm{L}$. Evidence for these target levels is weak in the neonatal population, however we have chosen to follow these targets until neonatal specific data is available. OBJECTIVE: Our previous dosing regimen frequently resulted in vancomycin trough levels $<10$ $\mathrm{mg} / \mathrm{L}$. This study was designed to assess the performance of our empiric dosing regimen in achieving therapeutic vancomycin trough levels, and to inform the creation of a revised empiric dosing regimen. Methods: Infants with a corrected gestational age 23-43 weeks, receiving vancomycin between January 2009 and December 2011, with at least one "pre" level were included. Demographic information, vancomycin doses and levels were collected. Where possible, pharmacokinetic parameters were calculated. The primary endpoint was the percentage of neonates that achieved measured pre vancomycin levels of $<10 \mathrm{mg} / \mathrm{L}, 10-20 \mathrm{mg} / \mathrm{L}$, and $>20 \mathrm{mg} / \mathrm{L}$ on the previous empiric dosing regimen. Secondary endpoints included the percentage of neonates that achieved extrapolated, true trough vancomycin levels in these ranges, the median total daily doses that were required to achieve target levels (10-20 $\mathrm{mg} / \mathrm{L}$ ), and the median total daily doses and dosing intervals that were pharmacokinetically predicted to achieve a trough level of $15 \mathrm{mg} / \mathrm{L}$. This information was used to devise a new empiric dosing regimen. RESULTS: Of the 158 infants who were started on our empiric regimen, $34.2 \%$ achieved an initial vancomycin trough level $10-20 \mathrm{mg} / \mathrm{L}, 65 \%<10$ $\mathrm{mg} / \mathrm{L}$, and $1 \%>20 \mathrm{mg} / \mathrm{L}$. There was a mean trough value of $8.7 \mathrm{mg} / \mathrm{L}$. Analysis of actual and predicted doses required to reach trough levels of $15 \mathrm{mg} / \mathrm{L}$ suggested increased empiric dosing for all neonatal age groups. We predict our revised regimen will result in $72 \%$ infants achieving target levels initially, with $12 \%$ below and $16 \%$ over target. We predict a mean trough of $15.4 \mathrm{mg} / \mathrm{L}$ on the revised regimen. CONCLUSIONS: Through examination of vancomycin levels achieved on our previous empiric dosing regimen, we determined that a dosage increase was necessary. Examination of the doses required and doses predicted to reach target levels informed a revised dosage regimen. This regimen will need to be clinically validated in an independent cohort in the future.

\section{THE IMPACT OF COMPUTERIZED PHYSICIAN ORDER ENTRY ON TOTAL PARENTERAL NUTRI- TION PRESCRIBING ERRORS IN THE NEONA- TAL INTENSIVE CARE UNIT \\ Christine Domonoske, ${ }^{1}$ April Graves, ${ }^{1}$ and Lakshmi Katakam ${ }^{2}$ \\ ${ }^{1}$ Children's Memorial Hermann Hospital, Houston, TX, USA, \\ ${ }^{2}$ The University of Texas Health Science Center at Houston, Division of Neonatal-Perinatal Medicine, USA christine. domonoske@memorialhermann.org}


INTRODUCTION: Patients in the neonatal intensive care unit (NICU) are vulnerable to medication prescribing errors due to weight-based dosing, frequent weight changes, and communication gaps. Total parenteral nutrition (TPN) orders in particular are prone to prescribing errors and can compromise patient safety. Previous studies demonstrated that implementation of computerized physician order entry (CPOE) allowed for safer and more efficient TPN ordering by decreasing the total number of TPN prescribing errors. The purpose of this study is to evaluate the impact of CPOE implementation on TPN-related prescribing errors in the NICU at Children's Memorial Hermann Hospital (CMHH). METHODS/DESIGN: This is a retrospective comparative cohort study of TPN errors before and after CPOE implementation. All TPNs prescribed in the CMHH's NICU, between 8/1/2012 and 9/13/2012, were reviewed for prescribing errors. Identified errors were categorized as the following: incomplete orders, electrolyte imbalances, dosing, calculation, calciumphosphorus ratio and transcription errors. The study was conducted over three distinctive, time periods. "Period A" consisted of the time prior to, "period B" consisted of the time during, and "period C" consisted of the time after CPOE implementation in the NICU. RESULTS: A total of 908 TPNs were prescribed during the study period, and 6.3\% (57/908) of these TPNs were noted to have errors. $37 \%(21 / 57)$ of the identified errors occurred during period A, 35\% (20/57) occurred during period B, and 28\% (16/57) occurred during period $C$. When simple logistic regression was used to compare the error frequency over the three time periods, the probability of TPN-related prescribing errors was slightly lower during period B (OR 0.83, 95\% CI 0.44-1.57) and period C (OR 0.82, 95\% CI 0.42-1.6), when compared to period A, but this difference was not statistically significant. However, the type of errors varied between the three time periods, with "incomplete orders" (10/21, 48\%) being the most frequent error during the pre-CPOE time period, "dosing errors" $(7 / 20,35 \%)$ being the most frequent error during CPOE implementation, and "electrolyte imbalances" (5/16, 31\%) being the most frequent type of error during the post-CPOE period. CONCLUSIONS: Implementation of CPOE did not significantly reduce the overall frequency of TPN prescribing errors in the NICU. However, the occurrence of particular types of prescribing errors may be changing with the continued utilization of CPOE. Continued surveillance of TPN orders is necessary to identify and address newly emerging trends in prescribing errors.

\section{QTC INTERVAL IN PEDIATRIC PATIENTS TREAT- ED WITH METHADONE}

Elizabeth Elson, Brandy Zeller, and Christopher McPherson

St. Louis Children's Hospital, St. Louis, MO, USAeelson@ bjc.org
INTRODUCTION: Methadone, a synthetic opioid analgesic, is utilized in pediatric patients for iatrogenic opioid withdrawal and pain management. Based on FDA MedWatch reports in adult patients, the package labeling was updated to include a black box warning addressing potentially increased QTC intervals and subsequent serious arrhythmias, specifically Torsades de Pointes. This is the first study designed to explore serial QTC interval measurements in pediatric patients treated with methadone therapy. METHODS: A retrospective chart review was conducted of pediatric patients admitted to St. Louis Children's Hospital between January 1, 2011 and June 30, 2012. Patients were included if they had a baseline electrocardiogram (ECG) and at least one ECG while on methadone therapy. For patients with multiple ECGs on methadone therapy, the ECG with the longest QTC interval was utilized for the primary analysis. Data was collected from the electronic medical record including demographic and clinical characteristics as well as QTC intervals from ECGs conducted at baseline and on therapy. The primary outcome compared the QTC interval on methadone therapy to the interval at baseline. Secondary outcomes included the incidence of QTC interval prolongation (QTC > $440 \mathrm{msec}$ ) on methadone therapy and identification of risk factors for QTC interval increases in this population. To identify risk factors, demographic and clinical characteristics were compared between patients with or without QTC interval increase on methadone therapy. The student's t-test and Mann Whitney U-test were utilized for parametric and nonparametric continuous variables, respectively. Chisquare test was used to compare binomial variables. RESULTS: Thirty-six patients were included in the study. The mean QTC interval measured during methadone therapy was increased from baseline $(474 \pm 51 \mathrm{vs}$. $454 \pm 41 \mathrm{msec}, \mathrm{p}=0.034)$. The high proportion $(47 \%)$ of the study population had increased QTC intervals during methadone therapy compared to baseline. Patients were more likely to have QTC interval prolongation during methadone therapy than at baseline $(75 \%$ vs. $56 \%$ ). Patients with QTC interval increases were more likely to have a history of arrhythmia ( 5 vs. 0 patients, $\mathrm{p}=0.016$ ) and were more likely to be on concomitant QTC prolonging medications, specifically fluconazole ( 4 vs. $0, p=0.04$ ). There was no difference between groups regarding total daily methadone dose $(0.32$ vs. $0.45 \mathrm{mg} / \mathrm{kg}, \mathrm{p}=0.551$ ), IV administration ( 2 vs. 1 , $\mathrm{p}=0.593$ ), or electrolyte abnormalities on methadone therapy (potassium: 4.2 vs. $4.0 \mathrm{mmol} / \mathrm{L}, \mathrm{p}=0.532$; magnesium: 2.0 vs. $2.0 \mathrm{mg} / \mathrm{dL}, \mathrm{p}=0.741$; calcium: 9.0 vs. $9.4 \mathrm{mg} / \mathrm{dL}, \mathrm{p}=0.274)$. No patients developed Torsades de Pointes during methadone therapy. CONCLUSION: A high proportion of pediatric patients on methadone therapy had evidence of QTC interval increases from baseline and QTC prolongation. Risk factors for development of QTC interval 
increases on methadone therapy included a history of arrhythmia and concomitant QTC prolongation medications, specifically fluconazole.

DOSING STRATEGIES AND SAFETY OF DEXMEDETOMIDINE IN NEONATES AND INFANTS Lauren Estkowski, ${ }^{1}$ Jennifer Morris, ${ }^{2}$ and Elizabeth Sinclair ${ }^{2}$

${ }^{1}$ Indiana University Health, Indianapolis, Indiana, USA, ${ }^{2}$ Riley Hospital for Children at Indiana University Health, Indianapolis, Indiana, USA lestkows@iuhealth.org

OBJECTIVE: The purpose of this study is to describe off-label use of dexmedetomidine, including dosing and duration, in neonates and infants in the pediatric intensive care unit (PICU). Patients were further evaluated for cardiovascular adverse events. Neonates and infants were compared to determine if differences in prescribing or safety existed. METHODS: Patients less than 12 months old with corrected gestational ages of at least 37 weeks receiving continuous infusion of dexmedetomidine at Riley Hospital for Children at Indiana University Health between October 2007 and August 2012 were assessed. Patients were excluded if dexmedetomidine was used for procedural sedation, post-cardiovascular surgery, or if post-surgery infusion weaning orders existed at the time of PICU admission. Demographics, vital signs, and concomitant receipt of antihypertensives, opioids, and other sedatives were collected retrospectively. Dosage information including dose range, duration, and indication was collected. Appropriate non-parametric tests were used for statistical analysis. RESULTS: A total of 127 patients, 28 neonates and 99 infants, who received dexmedetomidine during the study period were assessed. There was no difference in the median minimum dexmedetomidine dose between neonates and infants $[0.29 \mathrm{mcg} / \mathrm{kg} / \mathrm{hr}$ (IQR 0.18-0.31) vs. $0.2 \mathrm{mcg} / \mathrm{kg} / \mathrm{hr}$ (IQR 0.17-0.3), p=0.35]. In contrast, the median maximum dose was higher for infants than neonates $[0.6 \mathrm{mcg} / \mathrm{kg} / \mathrm{hr}$ (IQR 0.4-0.8) vs. $0.4 \mathrm{mcg} / \mathrm{kg} /$ $\mathrm{hr}$ (IQR 0.26-0.6), p=0.004]. Infusion duration was similar between the age groups [neonates: 38.5 hours (IQR 14.8-69.8) vs. infants: 42 hours (IQR 19-84), $\mathrm{p}=0.47]$. At least 1 episode of hypotension was noted in 34/127 ( $27 \%$ ) of the study population. There was no difference between neonates and infants in patients experiencing hypotension [4/28 (14\%) vs. 30/99 (30\%), p=0.15]. An episode of bradycardia was identified in 57/127 (45\%) patients; this occurred less frequently in neonates than infants $[2 / 28(7 \%)$ vs. $55 / 99(56 \%), p<0.01]$. Concomitant medications that could contribute to hypotension or bradycardia were used in 70/127 (55\%) of the study population; use was lower in neonates than infants [9/28 (32\%) vs. 61/99 (62\%), p<0.01]. Additional sedative agents were used in $90 / 127$ (71\%) patients, and use was less common in neonates than infants [15/28 (54\%) vs. 75/99 (76\%), $\mathrm{p}=0.02]$.
CONCLUSION: Dexmedetomidine dose ranges in this study were similar to the FDA-labeled dose for ICU sedation in adults. Dexmedetomidine was frequently used with other sedatives in neonates and infants in the PICU. More infants experienced a bradycardia episode than neonates, but they were also more likely to receive higher dosages of dexmedetomidine, additional sedatives, and concomitant medications that could contribute to cardiovascular adverse events. Larger, prospective studies are needed to further evaluate cardiac adverse events associated with dexmedetomidine use and to confirm a safe dosing range and duration of dexmedetomidine use in neonates and infants.

\section{IMPACT OF CLINICAL PHARMACIST'S INTER- VENTIONS ON DISCHARGE PROCESS IN A PEDIATRIC WARD}

Asmaa Ezz-Eldeen Mohamed, Hoda Badran, and Gavin Andrews

Hamad General Hospital, Doha, Qatar asmaa_ezz2005@ yahoo.com

BACKGROUND: Preparing to discharge pediatric patients is a challenging process as pediatric population has various factors that place them at a high risk of medication errors. It needs an effective synchronization between different health care providers to ensure safe and effective transition of care along with avoiding drug-related problems and adverse drug events after discharge. The objective of this study was to evaluate the role and effectiveness of clinical pharmacist's interventions on improving the safety of discharge process of pediatric patients. METHODS: It is a single- center prospective interventional study was conducted between December 2011 and May 2012 in Hamad General Hospital, a tertiary, teaching hospital in Doha-Qatar. The Clinical pharmacist's interventions were divided to prescription-related and patient-related interventions. Prescription-related interventions included the following: discharge medications reconciliation, review prescriptions against incomplete data and medication errors. Followed by patient -related interventions which were in the form of assessing parents' knowledge about their child's medications before and after clinical pharmacist counseling. The assessment was done through a pre-and postcounseling questionnaire to be filled by the parents. RESULTS: During the study period 52 pediatric patients were included. Medication reconciliation showed drug omission in $3.8 \%$ of the prescriptions. Medication errors were detected in $9.6 \%$ (5.8\% of errors were wrong doses while $3.8 \%$ were wrong frequencies). Allergy status of patients was missing in $42 \%$ of the prescriptions; weight was missing in $32 \%$, diagnosis in $17 \%$, duration in $7 \%$, and physician stamp in $2 \%$ of the reviewed discharge prescriptions. 
Clinical pharmacist's counseling significantly improved the parents' knowledge about indication, doses, frequency, side effects and appropriate way of administration of their child's medications ( $p$-value $<0.001)$. The majority of parents $(96.2 \%)$ documented that the counseling session is going to increase their adherence to the child's medications. Almost all parents $(98 \%)$ were satisfied and appreciated the provided service. Besides, overall rating of counseling session was "excellent" as per $92.3 \%$ of parents. CONCLUSION: Clinical pharmacist's interventions improved the safety of discharge process.This was shown through resolving discrepancies, correcting medication errors, completing missing data in discharge prescriptions, and finally improving parental overall knowledge about their child's discharge medications. Thus we recommend providing this service to all pediatric patients upon discharge.

DEVELOPMENT AND IMPLEMENTATION OF A COMPUTERIZED PRESCRIBER ORDER ENTRY (CPOE) PROCESS FOR SODIUM PHENYLACETATE/SODIUM BENZOATE AND ARGININE HCL FOR THE MANAGEMENT OF UREA CYCLE DISORDERS

M. Paige Fuller, ${ }^{1}$ Brendan Lanpher, ${ }^{2}$ Marshall Summar, ${ }^{2}$ and Fred Hargrove ${ }^{1}$

${ }^{1}$ Monroe Carell Jr. Children's Hospital at Vanderbilt, Nashville, TN, USA, ${ }^{2}$ Children's National Medical Center, Washington D.C., USA paige.fuller@vanderbilt.edu

BACKGROUND: Patients with urea cycle disorders (UCDs) have genetic defects in the metabolism of nitrogen generated by protein breakdown. Sodium phenylacetate/sodium benzoate (Ammonul) and arginine $\mathrm{HCl}$ are initiated in these patients emergently to provide an alternative pathway for excretion of excess nitrogen. Prompt initiation is essential as prolonged hyperammonemia can result in permanent neurological damage and death. The medication process for Ammonul and arginine $\mathrm{HCl}$ is complex, especially in infants and children. In 2009, the FDA released a review of 6 arginine $\mathrm{HCl}$ overdoses in pediatric patients, including 4 fatal errors. These medications prove cumbersome to order for many reasons. UCDs require defect-specific dosing strategies. Dosing regimens (mg $/ \mathrm{kg}, \mathrm{mL} / \mathrm{kg}, \mathrm{gm} / \mathrm{m} 2$, and $\mathrm{mL} / \mathrm{m} 2$ ) also vary according to weight and drug reference. Ammonul contains 2 salt forms, which makes transcribing doses challenging. Finally, these medications are packaged in adult-sized containers, further confusing their administration to pediatric patients. Although CPOE has been available within our hospital for some time, there was limited ordering support prior to implementation of the Ammonul/arginine $\mathrm{HCl}$ order pages. In the past, these orders were completed through a pharmacy miscellaneous process or written on blank order forms. METHODS/RESULTS: CPOE pages for Ammonul/ arginine $\mathrm{HCl}$ were developed for patients $\leq 20 \mathrm{~kg}$ and $>20 \mathrm{~kg}$. Patients are automatically routed according to dosing weight. Each page outlines indications that populate defect-specific loading and maintenance doses. A free-text option is available if needed for individualized dosing of arginine. Doses are provided as $\mathrm{mg} / \mathrm{kg}(\mathrm{mL} / \mathrm{kg})$ or $\mathrm{gm} / \mathrm{m} 2(\mathrm{~mL} / \mathrm{m} 2)$ based on medication and weight. Infusion times, rates, and start times for loading and maintenance doses are calculated and conveyed to nurses via the eMAR/barcoding system and medication label. "Call-for medication" reminders and compatibility information are provided for nurses. Preparation instructions, including standard dilution and diluent, final total volume, and a filter reminder for Ammonul are communicated to the pharmacy computer system from CPOE. Standardized drug files and order processing formats are in place within the pharmacy system. Also, medication guardrails were updated/added to smart pumps. Prior to final implementation, hospital staff were educated on the use of the new CPOE ordering pages and how they interact with other hospital systems. Since their implementation at the end of January 2010, the CPOE pages have been utilized in the management of 10 patients with UCDs during 13 separate hospital admissions. CONCLUSION: Implementation of the CPOE pages has provided a safe, standardized, and user-friendly medication ordering process for Ammonul and arginine $\mathrm{HCl}$ for the management of UCDs within our institution.

\section{DRUG UTILIZATION, DOSING AND COSTS AFTER IMPLEMENTATION OF INTRAVENOUS ACETAMINOPHEN GUIDELINES FOR PEDIAT- RIC PATIENTS}

Nicholas Fusco, ${ }^{1}$ Kristine Parbuoni, ${ }^{2}$ and Jill Morgan ${ }^{1}$ ${ }^{1}$ University of Maryland School of Pharmacy, Baltimore, MD, USA, ${ }^{2}$ University of Maryland Medical Center, Baltimore, MD,USAnfusco@rx.umaryland.edu

PURPOSE: The objectives of this study are to characterize the use of intravenous acetaminophen at our institution, determine if acetaminophen was prescribed at the age-appropriate dosing per institutional guidelines as well as evaluate compliance with restrictions for use. Total pharmacy costs associated with intravenous acetaminophen usage are described as well. METHODS: This was a retrospective, cohort study of pediatric patients $<18$-years-old, admitted to a tertiary care hospital who received at least one dose of intravenous acetaminophen between August 1, 2011 and January 31, 2012. Data were extracted from the electronic medical record, which included electronic medication administration record, operating reports, anesthesia records and discharge summaries. RESULTS: A total of 52 doses of intravenous acetaminophen were administered to 31 patients during the six-month study period. Most patients were 
admitted to the otorhinolaryngology service (55\%) and the majority of doses were either administered in the operating room $(46 \%)$ or the intensive care unit $(46 \%)$. Nineteen doses $(37 \%)$ of intravenous acetaminophen were administered to patients who did not meet the institutional guideline's eligibility criteria. Three patients received single doses of intravenous acetaminophen that were greater than the dose recommended for their age. One patient during the study period received more than the recommended 24-hour maximum cumulative dose for acetaminophen. Total drug cost of intravenous acetaminophen therapy over the six-month study period was $\$ 530.40$. CONCLUSION: Intravenous acetaminophen has been used most frequently among pediatric patients admitted to the otorhinolaryngology service during the perioperative period. Nineteen doses (37\%) were administered to patients that did not meet the institutional guideline's eligibility criteria. Reinforcing the availability of institutional guidelines will promote cost-effective use of intravenous acetaminophen, while minimizing the prescription of inappropriate doses.

INPATIENT PEDIATRIC PHARMACISTS PROVIDE TDAP IMMUNIZATIONS TO PARENTS AND CAREGIVERS OF CRITICALLY ILL INFANTS HOSPITALIZED AT CHILDREN'S HOSPITAL LOS ANGELES

Melanie George, Carol Taketomo, Lilly Yen

and Phuong Lieu

Children's Hospital of Los Angeles, Los Angeles, CA, USA mgeorge@chla.usc.edu

INTRODUCTION: Children's Hospital Los Angeles (CHLA) is located in Los Angeles, with $64 \%$ of the primary patient population being Hispanic. CHLA admits children from birth through 26 years of age for a variety of medical conditions. In 2010, the California Department of Public Health (CDPH) declared a pertussis epidemic in California. During that year, 9,156 cases of pertussis were reported in California, and 10 deaths were documented. Nine out of the ten deaths were Hispanic infants. 809 patients were hospitalized due to pertussis and $72 \%$ of those patients were infants less than 6 months old. $80 \%$ of those under 6 months that were hospitalized for pertussis were Hispanic. 1 To help control the epidemic, CDPH recommended Tdap administration to the following: all persons 10 years or older who had not received the Tdap booster; women of childbearing age, preferably before pregnancy or immediately after; all persons in close contact with infants, including family members and caregivers.2 The 2008 CDC survey showed that only $44 \%$ of adolescents in California and only $6 \%$ of adults nationwide had received their Tdap booster. 3 As a result of this epidemic and the predominant Hispanic patient population admitted to CHLA, inpatient pharmacists worked with the Infectious
Disease Department and CDPH to develop a protocol for pharmacists to offer free Tdap immunizations to parents and caregivers of critically ill patients $<6$ months of age who were admitted to the NICU and CTICU. By providing free Tdap immunizations, the inpatient pharmacists were able to help protect pediatric patients at the age where they were most susceptible and vulnerable to pertussis. METHODS: In collaboration with the Infectious Disease Department at CHLA and CDPH, a pharmacist administered Tdap protocol was developed, allowing immunization certified pharmacists to provide Tdap to parents and primary caregivers of patients $<6$ months of age admitted to the CTICU and NICU. Along with immunization certificates, pharmacists were also required to complete an annual CHLA competency prior to immunizing parents and caregivers. Informed consents were developed in English and Spanish, and parents/caregivers of patients $<6$ months of age were screened for eligibility criteria. Patients were identified daily during rounds, and vaccines were administered to parents/caregivers who were not previously immunized when the patient was either ready for discharge or transfer to the floor. RESULTS: Inpatient pharmacists were able to immunize 58 parents/primary caregivers between 11/2010-5/2012. No adverse drug events were noted during these immunizations. CONCLUSION: Immunizations are primarily conducted in an outpatient setting. This is a novel description of inpatient pharmacists providing Tdap vaccines to parents and caregivers of patients $<6$ months of age, thus protecting patients from pertussis during their most vulnerable age, when they have not yet been fully immunized.

\section{LOW CONCENTRATIONS OF LINEZOLID IN AN INFANT WITH MRSA MENINGOENCEPHALITIS Jennifer Girotto, ${ }^{1,2}$ Alberto Cohen-Abbo, ${ }^{1,2}$ and Nicholas Bennett $t^{1,2}$ \\ ${ }^{1}$ Connecticut Children's Medical Center, Hartford, CT, USA, ${ }^{2}$ University of Connecticut Schools of Pharmacy and Medicine, Storrs/Farmington, CT, USA jgirotto@ comckids.org}

Methicillin resistant Staphylococcus aureus (MRSA) meningoencephalitis is a rare condition in infants and one that can be associated with poor clinical outcome. We report a case of a 5 month old previously healthy boy who was successfully treated with vancomycin, linezolid, and rifampin for MRSA meningoencephalitis. The patient presented with fever, unresponsiveness, and bilateral periorbital edema. He was diagnosed with meningoencephalitis due to abnormal cerebral spinal fluid (CSF) findings and begun on acyclovir, vancomycin and ceftriaxone. ACT scan showed loss of grey-white differentiation in left middle cerebral artery (MCA) territory and was concerning for an infarct. The 
magnetic resonance imaging with angiogram showed a large stroke involving the MCA territory. His blood and CSF cultures were both positive for MRSA. Because of the poor outcomes associated with MRSA meningoencephalitis, and because it is often difficult to obtain adequate trough levels of vancomycin in infants, his treatment was changed to linezolid $30 \mathrm{mg} / \mathrm{kg} /$ day IV in 3 doses, rifampin $20 \mathrm{mg} / \mathrm{kg} /$ day IV in 2 doses and vancomycin. We were able to maintain vancomycin troughs between 17 and 25 with doses of 88-100 mg/ $\mathrm{kg} /$ day divided every 6 hours . Because of uncertain linezolid CSF penetration we obtained linezolid CSF and blood concentrations at the time the patient required a repeat lumbar puncture to evaluate MRSA clearance. The linezolid levels were drawn at 4 hours and 15 minutes after the 3rd doses of linezolid. Both levels were extremely low at $0.48 \mathrm{mg} / 1$ for the CSF and undetectable in the serum. Although the levels were very low the patient did clear the MRSA from the CSF. He remained on all three antibiotics for 2 weeks then discharged home on IV vancomycin and oral rifampin. Conclusion: Further research should be conducted to evaluate CSF concentrations of linezolid in sick infants and children. Our patient did well on his combination therapy, although the concentrations of linezolid appeared to be subtherapeutic.

\section{PHARMACIST'S ROLE IN MULTIDRUG RESIS- TANT INFECTION MANAGEMENT, THE CASE OF SAINTE-JUSTINE UHC'S NEONATAL INTENSIVE CARE UNIT}

Isabelle Goyer, Philippe Ovetchkine, Ahmed Moussa, Josianne Malo, Brigitte Martin, Maude Saint-Jean, and Yves Théoret

Sainte-Justine UHC, Montreal, Quebec, Canada isabelle. goyer@umontreal.ca

INTRODUCTION: Late-onset sepsis is a common cause of morbidity and mortality in the neonatal intensive care unit (NICU). Coagulase-negative staphylococci (CoNS) are the most frequently encountered pathogens, especially when a central line is present. Empiric treatment is vancomycin because reported CoNS resistance to cloxacillin ranges from $70 \%$ to $92 \%$. In Sainte-Justine UHC's NICU, a significant proportion of CoNS has become heteroresistant to vancomycin (increase in MICs and numerous treatment failures). A change in management was urgently needed. METHODS: A neonatologist and 2 microbiologists assessed the problematic and identified linezolid as a possible alternative treatment. A NICU clinical pharmacist was asked to join the team and help in the decision-making process. The team presented the gathered information and proposed management to the neonatologists, infectious disease physicians and microbiologists of Sainte-Justine UHC. The clinical pharmacist presented her literature review regarding the use of linezolid in neonates and suggested that therapeutic drug monitoring (TDM) should be done. Interdisciplinary meetings were held throughout the decision-making process. RESULTS: The team created an algorithm for the management of late-onset sepsis which was accepted throughout the hospital; it is now consistently used in the NICU. After reviewing the supporting evidence, the clinical pharmacist made recommendations regarding dosing and follow-up for linezolid. The proposed use was presented to the antibiotic stewardship committee, the pharmacology and therapeutics committee, the clinical pharmacokinetic unit and was discussed in multiple meetings with physicians and a pharmacologist. The clinical pharmacist created tools to help neonatologists and residents prescribe linezolid with appropriate follow-up, as well as tools to help nurses with administration. The pharmacist's recommendations were adopted, linezolid was added to the hospital formulary and the tools are now used by the NICU healthcare providers. The clinical pharmacist presented the evidence for TDM (risk of resistance emergence, risk of toxicity, narrow therapeutic index, correlation between efficacy/toxicity and blood levels, poorly known and unpredictable pharmacokinetics in NICU population, inter- and intra-individual pharmacokinetic variability) and made specific recommendations (number and timing of samples, as well as targets to aim for efficacy and safety). After discussion, the team accepted the suggestions and TDM was made available with the help of the clinical pharmacology laboratory. NICU clinical pharmacists are now interpreting TDM results and suggesting modifications when appropriate, in collaboration with physicians and the pharmacology laboratory. CONCLUSION: Linezolid has become, in specific indications, the cornerstone of late-onset sepsis treatment in Sainte-Justine UHC's NICU. Indications, follow-up and TDM were extensively discussed in multidisciplinary committees. The clinical pharmacist was an essential member of these committees and actively led the decision-making process by reviewing evidence and implementing new clinical practices in the NICU.

\section{IMPLEMENTATION OF RESPIRATORY SYN- CYTIAL VIRUS TREATMENT WITH INHALED RIBAVIRIN AND INTRAVENOUS PALIVIZUMAB IN PEDIATRIC BONE MARROW TRANSPLANT PATIENTS}

Ashley Greene and Zanette Kanani

Children's Hospital Colorado, Aurora, CO, USA ashley. greene@childrenscolorado.org

INTRODUCTION: Respiratory syncytial virus (RSV) causes upper respiratory tract infections such as rhinitis in addition to lower respiratory tract infections (LRTIs). Among immunocompromised patients, RSV more often causes LRTIs with mortality rates up to $80 \%$ without treatment. Synagis (palivizumab) is the only 
FDA-approved monoclonal antibody for prophylaxis of RSV. Virazole (ribavirin) is an inhaled synthetic nucleoside with antiviral activity and the only FDA-approved treatment for documented infections with RSV. An evaluation of the literature and published guidelines was performed. Favorable outcomes were noted throughout the literature in favor of dual therapy with palivizumab and ribavirin in the treatment of RSV in severely immunocompromised patients. At Children's Hospital Colorado (CHCo), it was decided to treat high-risk BMT patients based on the inclusion criteria in BMT clinical care guidelines. CASE REPORT: A 13 year old male with recurrent medulloblastoma was admitted for high dose chemotherapy with thiotepa and melphalan, followed by autologous BMT on January 21, 2013. A chest X-ray and viral PCR were obtained January 27 th due to a transient new oxygen requirement and cough. The cultures returned positive for RSV. Given his severe immunocompromised status, treatment was initiated with IV palivizumab and three times daily ribavirin per the upcoming BMT protocol. Palivizumab $15 \mathrm{mg} / \mathrm{kg}$ was given IV over 20 minutes for one dose on that same day. Ribavirin 2 grams was given by inhalation every 8 hours for a total of 15 doses. On February 1st, his cough was somewhat improved and thought to be more upper airway/mucositis than LRTI. Since he appeared to be clinically improving, a viral PCR was not obtained. He was discharged on February 13th without significant complications associated with his RSV infection. DISCUSSION: While there is limited data to support the use of inhaled ribavirin in combination with IV palivizumab, BMT patients have a poor chance of survival without these measures. Once infection invades the lung of a patient that is highly immunosuppressed, the likelyhood of survival diminishes dramatically. Although these medications are high in cost, they could be utilized to save patients' lives. For this reason, CHCo will begin treating patients meeting predefined criteria with a known RSV infection with both inhaled ribavirin and IV palivizumab in an attempt to improve outcomes in this high risk population. CONCLUSION: Beginning spring 2013, CHCo will not only screen for RSV infections in high risk BMT patients but will implement a supportive care guideline to improve outcomes in RSV infections within the immunocompromised patient population. Abundant research has been done in regards to the appropriate administration and timing of the medication. Guidelines from other children's hospitals have helped support the creation of CHCo's upcoming policy.

WARFARIN: A TALE OF TWO DRUG INTERACTIONS

Alison Grisso, Debra Dodd, and Julie Sinclair-Pingel Monroe Carell Jr, Children's Hospital at Vanderbilt, Nashville, TN, USA alison.grisso@vanderbilt.edu
INTRODUCTION: We report a case in which the international normalized ratio (INR) for warfarin increased in response to a short course of sildenafil and again to a single dose of fluconazole. Sildenafil is not listed as a drug interaction with warfarin in two widely used drug information sources and scant literature exists. While fluconazole is well documented to interact with warfarin when given over consecutive days, only one case series has been published as to its effects with a single dose. METHODS: A retrospective, observational case report was performed. It was reviewed and approved by our institution's IRB. Warfarin dosing, INR, sildenafil dosing, fluconazole dose and administration times were reported in an eleven year-old patient with cardiomyopathy awaiting heart transplant. Results: The patient was initiated on $15 \mathrm{mg}$ every 8 hours of sildenafil orally, with a dose increase after two doses to $20 \mathrm{mg}$. An immediate effect on the INR occurred as seen by an increase from 2.3 on day of initiation to a peak of 6 two days later. At this time, sildenafil was discontinued and the INR normalized. The patient received a total of four doses of sildenafil. Later in the patient's course of warfarin therapy, a single, $400 \mathrm{mg}$ oral dose of fluconazole was given for a yeast infection. The INR increased from 2 on the day of fluconazole administration to 3.9 on day six. Further warfarin doses were held and the INR peaked at 4.4 on day seven. Two days later, the patient received a heart transplant therefore the warfarin was not restarted. No other concurrent interacting medications were given at either time. No bleeding events were noted. CONCLUSIONS: Sildenafil is not a listed drug interaction with warfarin. All three medications are metabolized by the cytochrome P450 enzyme system. However, while sildenafil and warfarin are not strong inhibitors of each other, sildenafil is a weak inhibitor of CYP2C9 of which warfarin is a major substrate. Though multiple doses of fluconazole is a known interaction with warfarin, single doses can affect the INR as well. Careful consideration and monitoring are warranted with initiation of sildenafil on patients currently taking warfarin and vice versa.

\section{EVALUATION OF A GUIDELINE FOR MONITOR- ING PROPYLENE GLYCOL TOXICITY IN PEDIAT- RIC INTENSIVE CARE UNIT PATIENTS RECEIV- ING CONTINUOUS INFUSION LORAZEPAM}

Lizbeth Hansen, ${ }^{1}$ Rebecca Lange, ${ }^{2}$ and Sameer Gupta ${ }^{1,3}$ ${ }^{1}$ University of Minnesota Amplatz Children's Hospital, Minneapolis, MN, USA, ${ }^{2}$ Univeristy of Minnesota College of Pharmacy, Minneapolis, MN, USA, ${ }^{3}$ Univeristy of Minnesota Physicians, Minneapolis, MN, USA lizbethhansen@ gmail.com

OBJECTIVE: To determine the appropriateness of a guideline using osmol gap as an indicator of propylene glycol toxicity for pediatric pa- 
tients receiving continuous infusion lorazepam. METHODS: Based on data and recommendations for adult patients, guidelines were developed for the use of continuous infusion lorazepam in pediatric intensive care unit (PICU) patients at the University of Minnesota Amplatz Children's Hospital. A retrospective medical chart review was performed to assess the effectiveness of the guideline at monitoring for toxicity secondary to propylene glycol accumulation. Demographic and clinical data were collected in all PICU patients receiving continuous infusion lorazepam from February 2012 to September 2012. RESULTS: Twenty-one patients received continuous infusion lorazepam for sedation in the PICU during the nine-month study period for a total of 23 infusions. Fifteen patients had an osmol gap calculated at any point during the infusion. Eight (53.3\%) of the patients with an osmol gap calculated during lorazepam infusion had an elevated osmol gap defined as an osmol gap $\geq 12$. One patient with an elevated osmol gap experienced clinical toxicity, as indicated by elevated anion gap or lactate, while no patients without an elevated osmol gap experienced clinical toxicity. The average maximum lorazepam dose was $0.07 \mathrm{mg} / \mathrm{kg} /$ hour in patients with an elevated osmol gap and $0.05 \mathrm{mg} / \mathrm{kg} /$ hour in those without an elevated osmol gap. The average infusion duration in patients with an elevated osmol gap was 4.9 days while the average in those without an elevated osmol gap was 8.3 days. There were no patients with renal failure $(\mathrm{CrCl}<30 \mathrm{~mL} / \mathrm{min})$ included in the study. Of the five patients weighing $<5 \mathrm{~kg}$, one patient $(20 \%)$ had an elevated osmol gap while $7(70 \%)$ of the 10 patients $\geq 5 \mathrm{~kg}$ had an elevated osmol gap. CONCLUSIONS: Higher lorazepam dose appears to be an appropriate indicator for the need to monitor osmol gap in patients on continuous infusion lorazepam. Infusion duration was likely shorter in patients with an elevated osmol gap because these patients were transitioned to an alternative sedation regimen. Weight does not appear to be a predictor for risk of toxicity in the absence of other factors.

EVALUATING THE CURRENT DOSING GUIDELINE USED IN PEDIATRIC PATIENTS AT CHILDREN'S HOSPITAL COLORADO FOR CONTINUOUS INFUSION VANCOMYCIN

Amanda Hoffbeck and Michelle Zapapas

Children's Hospital Colorado, Aurora, CO, USA amanda. hoffbeck@childrenscolorado.org

INTRODUCTION: Vancomycin administered as a continuous infusion has become an attractive regimen for pediatric patients to obtain higher trough concentrations (goals 15-20 mg/L) with lower total daily doses. Following implementation of a continuous infusion vancomycin (CIV) guideline at Children's Hospital Colorado $(\mathrm{CHCO})$, it became apparent that starting doses of CIV were variable, primarily dependant on patient age. The purpose of this study was to evaluate the current dosing guideline and provide a more clear dosing regimen based on patient age. BACKGROUND: Pediatric patients typically exhibit greater clearance of vancomycin with shorter half-lives, thus making attainment of goal troughs of $15-20 \mathrm{mg} / \mathrm{L}$ more challenging. Recent literature supports CIV in the pediatric population at varying doses ranging from $40-60 \mathrm{mg} / \mathrm{kg} /$ day in order to circumvent the subtherapeutic troughs and nephrotoxicity seen with escalating doses of intermittent vancomycin therapy. $\mathrm{CHCO}$ implemented a CIV dosing guideline to dictate appropriate dosing and monitoring of CIV. These guidelines recommended target troughs of 15-20 mg/L for osteomyelitis, CNS infections, deep wound infections, and abscesses. Prior data analysis by $\mathrm{CHCO}$ for 22 patients revealed an appropriate CIV starting dose of $40 \mathrm{mg} / \mathrm{kg} /$ day. METHODS: This evaluation was a retrospective chart review assessing inpatients who received CIV from April 2009 to January 2013. Patients were included if they (1) received CIV at any time between April 2009 to January 2013, (2) had a goal trough concentration of $15-20 \mathrm{mg} / \mathrm{L}$, and (3) had appropriately drawn vancomycin serum concentrations reported. Patients were excluded if they (1) had a goal trough concentration other than 15-20 mg/L, (2) did not have appropriately drawn serum concentrations, and (3) did not continue on CIV long enough to obtain a serum concentration. For evaluation, the patient age, initial starting dose $(\mathrm{mg} / \mathrm{kg} /$ day), and final therapeutic dose were recorded. These parameters were evaluated in 3 patient age groups ( $<2$ years, 2-8 years, $>8$ years). RESULTS: There were a total of 33 patients evaluated after applying the inclusion/exclusion criteria. The average starting dose for patients in the $<2$ years of age group $(\mathrm{n}=5)$ was $48.9 \mathrm{mg} / \mathrm{kg} /$ day, and the average therapeutic dose was $50 \mathrm{mg} / \mathrm{kg} /$ day. In the 2-8 years of age group $(\mathrm{n}=11)$, the average starting dose was $40.5 \mathrm{mg} / \mathrm{kg} / \mathrm{day}$, and the average therapeutic dose was $47.8 \mathrm{mg} / \mathrm{kg} /$ day. For patients included in the $>8$ years of age group $(\mathrm{n}=17)$, the average starting dose was $37.6 \mathrm{mg} / \mathrm{kg} /$ day, and the average therapeutic dose was $39.4 \mathrm{mg} / \mathrm{kg} /$ day. CONCLUSION: This study, although limited in patient number, suggests that age-based dosing should be established for CIV initial dosing. These results are suggestive that more aggressive initial dosing (greater than $40 \mathrm{mg} / \mathrm{kg} /$ day) may be warranted for patients less than 8 years of age.

\section{APPROPRIATENESS OF ORAL MEDICATION DE- LIVERY DEVICES AVAILABLE IN COMMUNITY PHARMACIES}

Christina Phillips,${ }^{1}$ Allyson Votruba, ${ }^{1}$ Sajidah Swar, ${ }^{1}$ Michelle Condren, ${ }^{1,2}$ and Brooke Honey ${ }^{1,2}$ ${ }^{1}$ University of Oklahoma College of Pharmacy, Tulsa, OK, USA, ${ }^{2}$ University of Oklahoma School of Community Medicine, Tulsa, OK, USA brooke-honey@ouhsc.edu 
PURPOSE: Oral liquids remain a common dosage form used in children. However, they have been linked to medication administration errors, often from inappropriate/misused oral delivery devices. The authors recently published a study describing medication delivery devices found in community pharmacies that identified all devices contained markings in milliliters, with $79 \%$ also in teaspoons. Eighty-two percent of community pharmacies investigated carried 5-mL syringes, making this the most common device. This follow-up study identified the proportion of prescribed liquid medications that can be properly administered with devices available at local pharmacies. METHODS: Investigators selected five pharmacies within a five mile radius of the pediatric clinic. All clinic prescriptions over a two-month time frame were analyzed. Devices from the pharmacies were then compared with the prescriptions to determine if they were appropriate and/or optimal. Devices were deemed appropriate if the volume was able to be accurately measured and device units matched the prescription for that device. Data collected for each prescription included: presence of markings on the device at the prescribed dose, if the units of measurement matched the device, if appropriate to measure the prescribed volume with the given syringe, optimal syringe volume, and if the pharmacy had an appropriate device for the prescribed volume. RESULTS: Five community pharmacies, two national chains and three independents, were included in the study. Pharmacies carried on average 2.2 oral medication delivery devices each (range: 1-4). Among these 11 devices, 5 different types were found. All five pharmacies carried the 5-mL syringe marked in both $\mathrm{mL}$ and tsp, $80 \%$ of pharmacies stocked a $10-\mathrm{mL}$ device as either a syringe or spoon, and one pharmacy independently carried either a 1-mL or a 3-mL syringe. Over the two months of prescription data analyzed, 557 prescriptions were written with $158(28 \%)$ being liquids requiring a medication delivery device for administration. When comparing the 5 unique devices to 158 prescriptions independently, it was found that appropriateness was $10 \%, 28 \%, 36 \%$, and $65 \%$, respectively for the $1-\mathrm{ml}, 3-\mathrm{mL}, 5-\mathrm{mL}$, and $10-\mathrm{mL}$ devices. The 5 -mL syringe was optimal in only $22 \%$ of prescriptions analyzed and the $10-\mathrm{mL}$ syringe was found to be the most optimal device when compared to all prescriptions. Of the 5 pharmacies reviewed, all prescriptions could be appropriately measured using their devices $57 \%$ of the time (range: $36 \%-79 \%$ ). CONCLUSIONS: Oral medication delivery devices are imperative for safe and effective oral liquid medication use. The most commonly available $5-\mathrm{mL}$ syringe was found to be inappropriate for the majority of prescriptions written by a pediatric clinic; thus emphasizing the importance of device availability and education. Understanding optimal and appropriate devices will allow pharmacists to tailor patient-specific education and will allow direction when stocking oral delivery devices in the community pharmacy.

\section{ANTIMICROBIAL STEWARDSHIP PROGRAM (ASP) INITIATIVE: CURRENT USAGE OF ORAL VANCOMYCIN IN A TERTIARY CARE PEDIATRIC INSTITUTION}

Rachel Byerly, ${ }^{1}$ James Payne, ${ }^{1}$ Denise Howrie, ${ }^{1,2}$

Michael Green, ${ }^{2}$ and Marian Michaels ${ }^{2}$

${ }^{1}$ University of Pittsburgh School of Pharmacy, Pittsburgh, PA, USA, ${ }^{2}$ Children's Hospital of Pittsburgh of UPMC, Pittsburgh, PA,USA howrie@pitt.edu

INTRODUCTION: Antimicrobial stewardship programs (ASP) utilize targeted prospective monitoring as well as anti-infective agent restrictions to achieve costeffective drug use while reducing risk of drug resistance. For many years, use of oral vancomycin has required approval by the Infectious Disease Service (IDS). With increased severity and frequency of $C$. difficile infections (CDI) and anecdotal report of increased number of authorizations, a current profile of oral vancomycin use was needed in this pediatric tertiary care institution. METHODS: Under ASP drug use evaluation processes, a pharmacy student-initiated project was designed. Retrospective chart reviews were performed for pediatric patients who were identified through electronic health record technology to have received one or more oral vancomycin doses during the six month period from March through September 2012. Data collection included: underlying diagnosis(es), prescribing physician service, microbiologic testing including $\mathrm{C}$. difficile toxins, concurrent medications including proton pump inhibitors, anti-infectives, and immunosuppressants. RESULTS: Chart reviews identified 36 vancomycintreated patients in the 6-month period, including in-patients (18) and out-patients (18). The Gastroenterology Service was the most frequent prescriber $(53 \%)$ when compared to other pediatric $(28 \%)$ or surgical services $(19 \%)$ and was responsible for $83 \%$ of out-patient use. The Infectious Disease Service, although responsible for initial authorization of use for in-patients, was actively consulted for treatment decisions in only $28 \%$ of in-patients. Reasons for oral vancomycin use included: continued/recurrent diarrhea with previous CDI history (15), suspected moderate-severe CDI (6), suspected recurrent CDI in IBD (7), and empiric use in inflammatory bowel disease symptom flare (8). C. difficile testing showed toxin positivity in $55.6 \%$, negative toxin results in $27.8 \%$ and no test results documented in $16.7 \%$ patients (out-patients receiving empiric therapy). Relevant concurrent drug therapy included antibiotics $(72.2 \%)$, immunosuppressive agents (63.9\%) and PPIs (44.4\%). CONCLUSIONS: In hospitalized patients, oral vancomycin was used for documented CDI, usually in high risk patients often receiving concurrent risk drug therapies and met criteria for appropriateness of use. 
Most out-patients were IBD patients with acute symptoms flares with or without previous CDI history and under Gastroenterology Service care. Confirmatory CDI studies were lacking in several of these patients, often due to patient non-compliance with testing. However, empiric oral vancomycin use also appeared to be a treatment strategy used prior to augmentation of immunosuppression in this population. Opportunities for enhanced antimicrobial stewardship activities have been identified, especially for this patient population and physician service.

\section{MEDICATION ORDER ERRORS AFTER IMPLE- MENTATION OF COMPUTERIZED ORDERING IN THE NICU}

Franklin Huggins, ${ }^{1}$ Matthew Gebarski, ${ }^{3}$ Sara Ridges, ${ }^{2}$ and Craig Herzog ${ }^{2}$

${ }^{1}$ West Virginia University, Charleston, WV, USA, ${ }^{2}$ University of Utah, Salt Lake City, UT, USA, ${ }^{3}$ Home Health United, Madison, WI, USA frhuggins@hsc.wvu.edu

INTRODUCTION: Medication order entry errors are a significant source of medication misadventure in the pediatric population. Computerized prescriber order entry (CPOE) has been proposed as one method of reducing such errors. However, CPOE systems have the potential to introduce new types of errors into the medication order process and there are limited data on the effect of CPOE implementation in the neonatal intensive care unit (NICU). PURPOSE: To compare medication order entry error ratesina NICUbeforeand afterimplementation of CPOE. METHODS: We conducted a prospective observational trial examining all medication orders in a 48 bed, level IIIB, NICU before and after implementation of CPOE. A specialist NICU pharmacist reviewed and classified each order into one of 5 categories: 1) Correctly ordered. 2) Clinically inappropriate. 3) Incorrectly transcribed resulting in an incorrect entry on the medication administration record (MAR). 4) Correctly transcribed into the MAR but containing order entry errors which could result in inappropriate dispensing. 5) Errors introduced by the CPOE system itself. We then compared the frequency of orders with each type of error before and after implementation of CPOE. RESULTS: There were 2981 medication orders in the study period prior to CPOE and 2592 after. The overall order entry error rate dropped from $5.8 \%$ of all orders prior to implementation to $2.8 \%$ after $(R R=0.48$; $95 \%$ CI, 0.36-0.62). Clinically inappropriate orders increased from $0.4 \%$ of orders to $1.3 \%$ ( $R R=3.0 ; 95 \% C I$, 1.6-5.7). CPOE implementation resulted in a decrease in transcription errors $(0.077 \%$ vs $3.3 \%$; $R R=0.024$; $95 \%$ CI, 0.006-0.096), and dispensing errors ( $1 \%$ vs $2.2 \%$; RR $=0.45 ; 95 \% \mathrm{CI}, 0.29-0.70)$. Errors introduced by the CPOE system itself occurred in $0.4 \%$ of orders. No significant adverse drug events occurred as a result of an order entry error during the study period.
DISCUSSION: Our study confirms previously observed reductions in the number of medication order errors after implementation of $\mathrm{CPOE}$ but demonstrate an increase in some types of errors. Transcription errors were almost completely eliminated, occurring only when the pharmacist manually entered an order into the pharmacy system, a process which we discouraged. The decrease in frequency of orders which resulted in a correct entry on the MAR but which would not properly dispense reflects careful system design prior to implementation. The increase in the number errors in clinical decision making suggests that the system somehow facilitated selection of inappropriate medications, doses or routes of administration by prescribers. This suggests that more attention should be given to creation of decision support tools and education of providers when implementing CPOE. Careful design and post implementation monitoring are necessary to ensure that the CPOE system does not create unanticipated types of order errors.

\section{VALIDATION OF THE MEDICATION TAPER COMPLEXITY SCORE FOR METHADONE TA- PERS IN CHILDREN WITH OPIOID ABSTINENCE SYNDROME.}

Peter Johnson, ${ }^{1,2}$ Jamie Miller, ${ }^{1,2}$ Tracy Hagemann, ,,2 Christine Castro, ${ }^{1}$ and Donald Harrison ${ }^{1}$

${ }^{1}$ University of Oklahoma College of Pharmacy, Oklahoma City, OK, USA, ${ }^{2}$ University of Oklahoma College of Medicine, Oklahoma City, OK, USA peter-johnson@ouhsc.edu

INTRODUCTION: Methadone is commonly used to treat opioid abstinence syndrome (OAS) and is often prescribed as a taper schedule over days to weeks. Inadvertent dosing and/or administration errors by caregivers occurring with this schedule could lead to harm. The Medication Taper Complexity Score (MTCS) is a scoring tool with five categories that was developed to evaluate the complexity of home methadone tapers; a previous study demonstrated $95 \%$ inter-rater reliability. The purpose of this study was to validate the MTCS and determine if it is a reliable tool for clinicians to use to assess complexity of methadone tapers for OAS. METHODS: An expert panel of pediatric clinical pharmacists was convened and was provided nine methadone tapers of varying difficulty (i.e., "easy", "medium", and "difficult"). The primary objective was to further establish reliability and content/face validity of the MTCS in a broader group of clinicians. Content validity was assessed by comparing the MTCS scores between the nine tapers. A conference call was utilized to discuss scoring discrepancies and to discuss potential issues with the instrument. The secondary objective was to assess the instrument's reliability both within and between the nine tapers. Instrument reliability was assessed using a Chronbach's Alpha; with 0.8 as the minimum acceptable 
coefficient. Content (divergent) validity was assessed via a one-way ANOVA analysis (Bonferroni posthoc analyses) of the mean scores provided by panel members for the nine taper regimens. In addition to the overall reliability estimate, additional estimates were assessed for within and between nine tapers. Data analyses were conducting using Stata v10.1, with the a priori level of significance of $\mathrm{p}<0.05$. RESULTS: Six panel members were recruited, representing a range of geographical locations. They had a mean of $18.3 \pm 5.5$ years of experience. The panel included clinical pharmacists with practice expertise in general pediatrics, PICU, NICU, hematology/ oncology, and cardiology. The MTCS had an overall reliability coefficient of 0.9949 . There was a vivid discrimination between the "easy", "medium", and "difficult" tapers, $\mathrm{p}=0.001$. The panel recommended minor modifications to the MTCS. Overall, they felt that the MTCS was easy to use and there were potential applications of this tool for clinical practice and research. Finally, the reliability coefficients were statistically equal overall, between and within to those coefficients established in the instrument pre-test. CONCLUSIONS: The MTCS was found to be a reliable and valid tool to assess complexity of methadone tapers for OAS. This tool may be useful in assessing complex opioid tapers to target need for focused medication counselling. Further research should evaluate the association of the MTCS with clinical outcomes and caregiver perceptions of opioid tapers.

\section{MAXIMIZING PATIENT SAFETY THROUGH IMPLEMENTATION OF A FORMALIZED PAR- ENTERAL POTASSIUM COMPOUNDING PRO- CEDURE}

Lauren Kattner, Kelli Crowley, Carol Vetterly, Don Berry, and Jeffrey Goff

Children's Hospital of Pittsburgh of UPMC, Pittsburgh, PA,USAlkatt23@gmail.com

BACKGROUND: It is the responsibility of medical professionals to ensure the safety of patients. Potassium salts have been identified by both the Institute for Safe Medication Practices and The Joint Commission (TJC) as high risk parenteral additives. Various safety precautions have been mandated by TJC, such as removal of concentrated potassium products from floor stock on patient care units and instituting computerized physician order entry. The role of the pharmacy department has been to safely compound and dispense intravenous (IV) products containing potassium; however, there are limited preparation method recommendations. The pharmacy department has determined that instituting a protocol that includes safer practices for the pharmaceutical compounding of IV potassium-containing products is a key component for proactively preventing serious medication errors. METHODS: A comprehensive literature search and phone surveys of seven pediatric hospitals were conducted; it was discovered that there is a lack of specific compounding procedures for the preparation of potassium-containing parenteral products. A collaborative team of clinical and distributive pharmacists, technicians, and pharmacy administration formulated precise potassium compounding procedures to be incorporated into the existing standard IV compounding process which includes additional pharmacist verification at multiple points of the preparation. Additionally, strengthening of the procedure through technician training/education, color-coded productspecific labeling for products containing potassium chloride (yellow) and potassium phosphate (orange), and emphasis on potassium product segregation has been integrated. Efficacy of the protocol was measured via adverse events reported in RiskMaster and with chemical analysis of three random samples collected every month from parenteral potassium preparations. RESULTS: These processes were put into operation March 13, 2012. There has been no Patient Safety Occurrence Reports involving potassium documented in RiskMaster in the time period between 3/13/12 and present. Thirty-seven infusion samples were collected and analyzed between 3/18/12 and 2/28/13. Variance between laboratory analysis values and intended values ranged between $-13 \%$ and $+7.7 \%$ with a mean of $-5.6 \%$ [IQR $-9.8 \%$ to $-3.85 \%$ ]. This is felt to be acceptable ranges when accounting for overfill volumes routinely added by manufacturers. There is no pre-implementation data to compare these values to, but it may be presumed that potential adverse medication events have been prevented due to increased control over compounding processes. CONCLUSION: Implementation of a standardized process for preparation of IV potassium products is an important step toward fulfilling our commitment to accurate compounding practices of this electrolyte and improved safety of pediatric patients. A formalized IV potassium compounding procedure with heightened awareness may provide less opportunity for errors to occur.

\section{THE USE OF CLINICAL DEBATES AS AN ACTIVE-} LEARNING TOOL IN TWO ELECTIVE COURSES Kristin Klein, ${ }^{1,2}$ Allison Blackmer, ${ }^{1,2}$ and Heidi Diez ${ }^{1}$ ${ }^{1}$ University of Michigan, College of Pharmacy, Ann Arbor, MI, USA, ${ }^{2}$ University of Michigan Health System, Ann Arbor, MI, USA kriklein@umich.edu

OBJECTIVE: The purpose of this poster is to describe the clinical debate process implemented in two elective courses (Immunizations and Pediatrics), and to discuss the results of a quality improvement assessment of students' attitudes and perceptions regarding the debates. DESIGN: During the 2011-2012 academic year, clinical debates were implemented in the Immunizations and Pediatrics electives as an active-learning 
strategy to allow students to self-teach controversial topics when paired with group discussions. Students enrolled in either or both electives were asked to complete a survey regarding their attitudes towards achieving the goals of the debate (e.g., improved knowledge of the topic, improved critical thinking skills, improved ability to defend opinions). The survey contained a total of seventeen questions; thirteen questions related to the goals of the exercise using a Likert scale, and four open-ended questions asking students to address the following: overall benefit of the exercise, positive aspects of the debate, areas of needed improvement, and additional comments. RESULTS: A total of 37 students were enrolled in either or both elective courses. Twenty-four of those students completed the survey. The students' responses were de-identified prior to analyzing the survey results. Seventy-five percent of the students who responded found the debate to be a beneficial experience overall. At least $50 \%$ of the students surveyed responded positively (i.e., agree or strongly agree) towards seven of the thirteen objectives assessed. Two objectives (improved abilities to perform literature searches and to analyze medical literature) received neutral scores from approximately $50 \%$ of the students, although these areas were also listed frequently by some respondents as one of the most beneficial aspects of the exercise. Other aspects identified as most beneficial included learning how to defend a clinical position, improving the ability to think on the spot, and working collaboratively in a team. Some areas of improvement identified by students included providing more instruction on how to conduct a debate, and allowing students to pick their groups or topics of debate. CONCLUSIONS: Overall, the debate was well received by the students who responded to the survey. Students responded that the debate improved their abilities to think on the spot, create a persuasive argument, work as a team, and communicate effectively. As a result of the successful implementation of the clinical debates in the Immunizations and Pediatrics electives, several other courses at our institution have implemented clinical debates as an active-learning tool.

\section{A FILTER THAT REDUCES ALUMINUM, A CON- TAMINANT IN CALCIUM GLUCONATE FOR INJECTION USP, IN PARENTERAL NUTRITION SOLUTIONS FOR PEDIATRIC PATIENTS}

Robert Kuhn, ${ }^{1,3}$ Wesley Harris, ${ }^{2,3}$ Christopher Spilling, ${ }^{2,3}$ Vasiliy Abramov, ${ }^{4}$ Jason Lone, ${ }^{4}$ and Robert Yokel ${ }^{1,3}$

${ }^{1}$ University of Kentucky College of Pharmacy, Lexington, $K Y$, USA, ${ }^{2}$ University of Missouri at St. Louis, St. Louis, MO, USA, ${ }^{3}$ Alkymos Inc, Lexington, KY, USA, ${ }^{4}$ Occam Design, Louisville, KY, USA rjkuhn1@email.uky.edu

INTRODUCTION: Aluminum is a contaminant of large and small volume component solutions used to compound parenteral nutrition solutions. Alumi- num produces no beneficial effects in the human. It can produce cognitive impairment, metabolic bone disease, and may contribute to cholestasis in patients receiving total parenteral nutrition. Neonates are the population at greatest risk for aluminum toxicity due to their immature renal function (the primary route of aluminum elimination) and high calcium requirement. Calcium Gluconate Injection, USP, contributes $\sim 85 \%$ of the aluminum in parenteral nutrition solutions. Typical parenteral nutrition solutions deliver 15 to $23 \mathrm{mcg} \mathrm{Al} / \mathrm{kg} /$ day to 4 to $6 \mathrm{~kg}$ and $<1 \mathrm{~kg}$ patients, respectively (Poole et al, JPGN, 2010) well above the 4 to $5 \mathrm{mcg} / \mathrm{kg} /$ day considered non-toxic in the FDA's labeling requirement that addresses aluminum in large and small volume parenterals (21CFR201.323). Currently there are no ways to remove aluminum from calcium salts prior to preparation of pediatric or neonatal TPN. OBJECTIVE: To reduce aluminum delivered to neonates via total parenteral nutrition to a non-toxic level. METHODS: A flow-through filter containing an immobilized chelator that complexes aluminum from calcium gluconate as it flows through the filter was designed and extensively tested. The filter and vial containing a partial vacuum that receives the filtered calcium gluconate solution comprise everything needed. RESULTS: The extent of aluminum removal is flowrate dependent. At a flow rate of $\sim 1 \mathrm{~mL} / \mathrm{min} \geq 90 \%$ of the aluminum is removed from calcium gluconate that contains up to $\sim 12,000 \mathrm{mcg} \mathrm{Al} / 1$. This is calculated to reduce aluminum delivered to $>1 \mathrm{~kg}$ infants to $<5$ $\mathrm{mcg} / \mathrm{kg} /$ day.

CONCLUSIONS: A point-of-use self-contained aluminum-complexing filter has been created. It reduces aluminum delivered in total parenteral nutrition solutions by $\sim 75 \%$, resulting in daily aluminum delivery below the level considered toxic to all but the smallest $(<1 \mathrm{~kg})$ infants.

\section{HEMODYNAMIC EFFECTS OF DEXMEDETOMI- DINE IN 82 INFANTS LESS THAN TWO MONTHS OF AGE}

Rhonda Kurz, Vanessa Holton, Paul Bakerman, Paul Liu, Lilanthi Balasuriya, and Brianne Wolfe Phoenix Children's Hospital, Phoenix, AZ, USA rkurz@ phoenixchildrens.com

PURPOSE: Appropriate selection of sedative and analgesic pharmacologic agents remains a challenge in pediatric and neonatal critical care. Dexmedetomidine (DEX) has become an attractive sedative option; however, little is known about its safety in very young infants. Due to DEX's potential effects on hemodynamics, the objective was to describe the effects on mean arterial pressure (MAP) and heart rate (HR) during the first 12 hours of DEX infusion in infants less than 2 months of age. METHODS: A retrospective chart review was performed for infants less than 2 months of age who re- 
ceived continuous infusions of DEX between October 2008 and December 2011 in a critical care setting (CVICU, PICU, and NICU) at a tertiary, university-affiliated, children's hospital. Data collected included patient demographics, intubation status, presence of an arterial line, and DEX infusion characteristics. Vasoactive-inotropic score (VIS), MAP and HR values were recorded at baseline, 6 and 12 hours. Initiation and discontinuation of DEX infusion was at the discretion of the practitioner; there was no predetermined protocol for sedation. RESULTS: During the 39-month study period, 93 DEX infusions were reviewed. Patient weight was $1.4-5.8 \mathrm{~kg}$ (mean $3.6 \mathrm{~kg}$ ) and age 3-62 days (mean 28 days). The average DEX infusion starting dose was $0.30 \mathrm{mcg} /$ $\mathrm{kg} / \mathrm{hr}$ and $0.37 \mathrm{mcg} / \mathrm{kg} / \mathrm{hr}$ at 6 and 12 hours. Baseline MAP for all infusions was $60.5 \mathrm{mmHg}$ and decreased by $16.9 \%$ to $50.3 \mathrm{mmHg}$ at 6 hours $(\mathrm{p}<0.001)$ and by an additional $2.8 \%$ to $48.6 \mathrm{mmHg}$ at 12 hours $(\mathrm{p}=0.0041)$. Baseline HR was 151 beats per minute (bpm) and decreased by $10.6 \%$ to $135 \mathrm{bpm}$ at 6 hours $(\mathrm{p}<0.001)$ and by an additional $5.2 \%$ to $128 \mathrm{bpm}$ at 12 hours $(\mathrm{p}<0.001)$. In 52 DEX infusions with concomitant vasoactive agents, there was no escalation of support based on VIS values. A surgical subgroup of 19 patients had a greater decrease in MAP at 6 hours compared to the overall study population, despite comparable baseline values. DEX was discontinued in 13\% (12/93) infusions during the study period, with these patients experiencing a more profound decrease in MAP. Eight in the discontinued group had recent cardiovascular surgery and were receiving nitroprusside and milrinone concurrently. CONCLUSIONS: There was a statistically significant decrease in MAP and HR during the observation period; however, patients on vasoactive agents prior to initiation of DEX did not require escalation of inotropic support. Patients that had DEX discontinued were more likely to have had recent cardiovascular surgery and be receiving nitroprusside and milrinone concurrently. Clinicians should be aware of the potential hemodynamic effects of DEX and close monitoring is warranted. Despite these effects, in the majority of infants less than 2 months of age, DEX was well tolerated and effects on MAP and HR were clinically insignificant.

\section{ASSOCIATION OF CONTINUOUS INFUSION FENTANYL WITH NEURODEVELOPMENTAL OUTCOMES IN VERY LOW BIRTH WEIGHT INFANTS}

Erin Lammers, ${ }^{1}$ Peter Johnson, ${ }^{2,3}$ Kimberly Ernst, ${ }^{3}$ Tracy Hagemann, ${ }^{2,3}$ Shelley Lawrence, ${ }^{3}$ Patricia Williams, ${ }^{3}$ Michael Anderson, ${ }^{4}$ and Jamie Miller ${ }^{2,3}$ ${ }^{1}$ University of Missouri Healthcare, Columbia, MO, USA, ${ }^{2}$ University of Oklahoma College of Pharmacy, Oklahoma City, OK, USA, ${ }^{3}$ University of Oklahoma College of Medicine, Oklahoma City, OK, USA, ${ }^{4}$ University of Oklahoma Public Health, Oklahoma City, OK, USA lammersem@ health.missouri.edu
INTRODUCTION: Fentanyl and other opioid analgesics are commonly used in the neonatal intensive care unit (NICU) as a continuous infusion (CI) for postoperative pain and sedation with prolonged mechanical ventilation. Fentanyl CIs have been associated with negative short-term effects (e.g., reduced brain size), but long-term neurodevelopmental effects have not been evaluated. The purpose of this study is to determine the association of long-term neurodevelopmental effects with fentanyl exposure. METHODS: This is a descriptive, retrospective study of very low birth weight $(\leq 1.5 \mathrm{~kg})$ neonates who received follow-up in the premature infant development clinic between January 2008 to December 2011 following discharge from the institution's 88-bed NICU. Data collection included demographics (e.g., gestational age, birth weight), measurements of acuity (e.g., Clinical Risk Index for Babies [CRIB II] score, duration of mechanical ventilation, length of stay), Bayley Scale of Infant and Toddler Development, Third Edition (Bayley-III) composite scores, and dose and duration of fentanyl and midazolam. The primary objective was to determine the relationship of fentanyl CI dose $(\mathrm{mcg} / \mathrm{kg})$ to each Bayley-III composite score for language, cognition, and motor skills. The secondary objective was to compare mean Bayley-III composite scores between treatment groups subdivided based on fentanyl CI dose and duration. The primary statistical analysis included three separate multiple linear regression models with each Bayley-III score as the dependent variables. Independent variables included CRIB II score, ventilator days, and fentanyl cumulative dose $(\mathrm{mcg} / \mathrm{kg})$ with a p value $<0.05$. RESULTS: A total of 208 patients were identified within the study period; however, only 147 patients were included in the study analysis. The mean gestational age and birth weight were $29 \pm 2.2$ weeks and $1153 \pm 251$ grams, respectively. Thirty-two $(21.8 \%)$ patients received fentanyl CI at a median cumulative dose of $226 \mathrm{mcg} / \mathrm{kg}$ (6-1945) and duration of 195 hours (2-898). There was no statistically significant correlation observed between fentanyl cumulative dose and any of the three evaluated Bayley-III composite scores. Significant associations were noted between each composite score and two independent variables, sex and follow-up frontooccipital circumference. In addition, the use of vasopressor agents was significantly correlated with language and motor skills composite scores. The diagnosis of bronchopulmonary dysplasia was also correlated to the composite score for motor skills. CONCLUSIONS: Although negative effects have been identified with the use of sedative and analgesic agents in the short-term, this study found no correlation between cumulative fentanyl dose and evaluated Bayley-III composite scores. An optimal balance between adequate analgesia and sedation and the avoidance of potential short-term and long-term consequences of using CI opioids is required. Further 
studies are needed to provide a better understanding of the risks versus benefits of sedation and analgesia practices in premature neonates.

DRUG UTILIZATION REVIEW OF LEVOSIMENDAN AT A MOTHER-CHILD UNIVERSITY HEALTH CENTER, MONTREAL CANADA

Annie Lavoie, ${ }^{1,2}$ and Marie-Laurence Parent-Blais ${ }^{2}$ ${ }^{1}$ CHU-Ste-Justine, Montreal, Canada, ${ }^{2}$ Faculty of Pharmacy, University of Montreal, Montreal, Canada annie. lavoie.hsj@ssss.gouv.qc.ca

INTRODUCTION: Levosimendan exerts positive inotropic effects by enhancing troponin-C affinity for calcium, thus facilitating myosin sliding and shortening of myofilaments. In addition, it acts on ATP-sensitive potassium channels to produce peripheral vasodilation. This drug is active for many days despite a $24 \mathrm{~h}$ infusion, because the half-life of active metabolite is 80-90 hours. Levosimendan is approved as a second-line agent in Asia, Australia, Europe and South America in acute decompensated cardiac failure. Some studies have been published regarding its use in pediatric patients but evidence showing its superiority compared to milrinone or dobutamine is still lacking. Since levosimendan is not approved in Canada, Health Canada Special Access Program (HCSAP) authorization had to be obtained. Because levosimendan is expensive, difficult to acquire, and because the experience of our team with this drug is limited, a drug utilization review (DUR) was pertinent. The goal of this DUR was to describe the population to whom the medication was prescribed and how it was used. METHODS: We performed a DUR of Levosimendan used in the pediatric intensive care unit from a motherchild university health center. Patients who received levosimendan were identified using the pharmacy software and data was collected from their medical charts. A standardized DUR data collection sheet was adapted for this drug. We collected demographics, levosimendan dosage, frequency and number of infusions, concomitant treatments, daily morning mean arterial pressure, pro-BNP, mixed venous saturation, blood urea nitrogen (BUN), creatinin and, diuresis. RESULTS: Twelve patients with a median [min-max] age of 25.5 [0.4-168] months received levosimendan. Their diagnosis was restrictive cardiomyopathy (1), hypertrophic cardiomyopathy (1), dilated cardiomyopathy (5), and congenital heart disease (5). They were given a median [min-max] of 1 [1-23] infusions. The mean total dose received per infusion per patient was 354.3 [45.0-552.0] $\mathrm{mcg} / \mathrm{kg}$ and no boluses were given at the beginning of the perfusion. When more than one infusion was given, the mean interval between infusions was of 7.3 [6.4-8.9] days. Levosimendan was stopped for a possible adverse reactionin one patient, to implement LVAD in 3 patients, because of stabilisation and discharge from ICU in 3 patients, and because the HCSAP authorization had expired in one patient. Four patients died because of their disease. Mean arterial blood pressure did not vary significantly during the time of infusion except for one infusion. Results in terms of laboratory values and concomitant treatments will be added. CONCLUSIONS: For the time being, it is not possible to obtain levosimendan from Health Canada Special Access Program anymore. Literature reports the use of 24-hour infusions and data is lacking for repeated infusions. Our experience shows minimal hemodynamic impact and depicts the use of repeated infusions longer than $24 \mathrm{~h}$. This underlines the importance to establish local guidelines.

\section{EVALUATION OF AN INSULIN THERAPY PRO- TOCOL IN VERY LOW BIRTH WEIGHT INFANTS IN A NEONATAL INTENSIVE CARE UNIT (NICU) Caren Liviskie, ${ }^{1}$ Christopher McPherson, ${ }^{1,2}$ and Brandy Zeller $^{1}$ \\ ${ }^{1}$ St. Louis Children's Hospital, St. Louis, MO, USA, ${ }^{2}$ Wash- ington University School of Medicine, St. Louis, MO, USA cliviskie@bjc.org}

INTRODUCTION: Hyperglycemia commonly occurs in very low birth weight (VLBW) neonates and is associated with morbidity and mortality. The treatment of hyperglycemia with insulin often results in hypoglycemia, a complication also associated with increased mortality. The purpose of this study is to evaluate the functionality, efficacy and safety of a conservative insulin therapy titration protocol for the management of hyperglycemia in VLBW neonates. METHODS: A retrospective chart review was performed including all VLBW neonates admitted to the NICU at St. Louis Children's Hospital who received insulin between July 1, 2008 and August 1, 2012. Data was collected from electronic medical records including demographics, insulin and glucose infusion rates (GIR), blood glucose levels prior to and during insulin infusion and concurrent medications. The primary outcomes were to compare glucose levels between the protocol compliant and non-compliant groups and to determine the number of hypoglycemic events in each group. Secondary outcomes included the amount of insulin required, GIR, average blood glucose and time to attainment of normoglycemia between the two groups. Neonates treated in a manner compliant with the protocol were compared to those treated in a non-compliant manner utilizing student's t-test and the Mann-Whitney U-test for parametric and non-parametric continuous variables, respectively. RESULTS: Ten neonates with a total of 13 insulin infusion initiations were included. Six of the 13 (46\%) insulin infusions were treated in a manner noncompliant with the protocol. The average GIR was similar between groups and remained constant throughout the insulin infusion ( 4.6 vs. $5 \mathrm{mg} / \mathrm{kg} / \mathrm{min}, \mathrm{p}=0.519$ ). 
The neonates in the noncompliant group had significantly higher blood glucoses at nine hours (342 vs. $212 \mathrm{mg} / \mathrm{dL}, \mathrm{p}=0.029$ ) and 12 hours ( 289 vs. 166 $\mathrm{mg} / \mathrm{dL}, \mathrm{p}=0.049)$. The noncompliant protocol group had significantly higher insulin infusion rates at six hours (0.03 vs. 0.02 units $/ \mathrm{kg} /$ hour, $\mathrm{p}=0.032)$, nine hours ( 0.03 vs. 0.01 units $/ \mathrm{kg} /$ hour, $\mathrm{p}=0.005)$ and 12 hours ( 0.04 vs. 0.01 units $/ \mathrm{kg} /$ hour, $\mathrm{p}=0.020)$. Only two hypoglycemic events occurred. Both occurred in the same neonate managed in a non-compliant manner. There was no difference found between the noncompliant and the compliant protocol group in the time to goal blood glucose (15.3 vs. 14.7 hours, $\mathrm{p}=0.935$ ) and average blood glucose during the insulin infusion ( 141 vs. $148 \mathrm{mg} / \mathrm{dL}, \mathrm{p}=0.737$ ). CONCLUSIONS: A conservative insulin therapy titration protocol for the management of hyperglycemia in VLBW neonates resulted in the safe attainment of normoglycmia, even with minor protocol violations.

\section{RESPIRATORY VIRAL INFECTION IN CHIL- DREN: POTENTIAL LINKS BETWEEN ADENO- VIRUS AND ASTHMA}

Jason Weinberg, ${ }^{1,3}$ Kristin Klein, ${ }^{1,2}$ Krystal Sheerer, ${ }^{1,2}$ and Adam Loyson ${ }^{1,2}$

${ }^{1}$ University of Michigan Health System, Ann Arbor, MI, USA, ${ }^{2}$ University of Michigan College of Pharmacy, Ann Arbor, MI, USA, ${ }^{3}$ University of Michigan Medical School, Ann Arbor, MI, USA loysonad@umich.edu

BACKGROUND: Respiratory viral infection (RVI) is a major trigger for wheezing episodes in infants and asthma exacerbations in children. Respiratory syncytial virus and rhinovirus infections during infancy have been associated with increased risks of asthma later in childhood; however, little is known about long-term effects of early adenovirus (Ad) infection and outcomes, such as asthma. OBJECTIVE: We sought to determine if early Ad infection increased the risk of developing asthma. DESIGN/METHODS: We retrospectively analyzed results of all assays for respiratory viruses (e.g., viral cultures, antigen testing, polymerase chain reactions) in patients $<18$ years of age performed from October 2000 to September 2010. Electronic medical records for patients with respiratory virus testing were queried for ICD-9 codes corresponding to adenovirus, respiratory syncytial virus, bronchiolitis, viral pneumonia, asthma/RAD, cough and wheezing. We compared the incidence of asthma in children with Ad infection identified during the first two years of life to the incidence in a control group of children matched by age who had negative respiratory tests during the same time period. RESULTS: Respiratory virus testing data were available for between 440 and 1206 pediatric patients per year. A virus was identified in $24.5-36.3 \%$ of tested patients each year. Ad accounted for 1.2 to $11.3 \%$ of positive tests each year. Asthma was eventually diagnosed in 23 of 55 children $(41.9 \%)$ in whom Ad was detected in the first two years of life compared to 29 of 110 children (26.4\%) whose respiratory viral tests were negative. There were no statistical differences between groups in birth weight or prematurity. Compared to children with negative tests, children in whom Ad was detected during the first two years of life were more likely to be in daycare $(2.528 \mathrm{OR}$, $1.066-5.99795 \% \mathrm{CI}, \mathrm{p}=0.035)$ or exposed to pets at home (3.193 OR, 1.506-6.769 95\% CI, p=0.003). Children who had been infected with Ad were more likely to develop asthma later in life than were children whose respiratory viral tests were negative $(2.008 \mathrm{OR}$, $1.012-3.97695 \% \mathrm{CI}, \mathrm{p}=0.046 ; \mathrm{X} 2=4.058, \mathrm{df}=1, \mathrm{p}=0.044)$. CONCLUSIONS: Ad was detected in a relatively small but consistent percentage of patients each year. Early Ad infection increased the likelihood of being diagnosed with asthma later during childhood, although it is possible that factors such as daycare or pet exposure could contribute to this effect. With ongoing work, we will more specifically define the independent contributions of Ad infection to asthma pathogenesis.

\section{TARGETING HIGHER VANCOMYCIN TROUGH LEVELS IN THE NEONATAL INTENSIVE CARE UNIT}

Jacqueline Magers, ${ }^{1}$ Kristen Gawronski, ${ }^{2}$ and Lindsay Schray ${ }^{1}$

${ }^{1}$ Nationwide Children's Hospital, Columbus, OH, USA, ${ }^{2}$ The Ohio State Univeristy Wexner Medical Center, Columbus,OH,USA jacqueline.magers@nationwidechildrens.org

INTRODUCTION: Methicillin-resistant Staphylococcus aureus (MRSA) has become a common problem in healthcare systems across the United States. Due to the increase in MRSA's prevalence, vancomycin use has also increased. Given conflicting reports of actual toxicity caused by vancomycin itself (and not impurities found in earlier preparations), a consensus guideline was released in 2009 to help guide the therapeutic monitoring of vancomycin in adults. This guideline recommends trough serum vancomycin concentrations should always be maintained above $10 \mathrm{mg} /$ $\mathrm{mL}$ to avoid development of resistance. The primary objective of this study was to develop an empiric dosing regimen in neonates that will obtain a vancomycin trough concentration between $10-20 \mathrm{mg} / \mathrm{mL}$. Methods: A retrospective chart review was conducted from January 1, 2009-June 30, 2009 of all Neonatal Intensive Care Unit (NICU) patients at Nationwide Children's Hospital (NCH) who received scheduled vancomycin and had at least one trough concentration obtained. Exclusion criteria: one-time vancomycin dose and level, inappropriately timed level or cystic fibrosis. From this data an empiric dosing algorithm 
was created and implemented (Algorithm \#1). To assess the ability to attain the targeted concentration of $10-20 \mathrm{mcg} / \mathrm{ml}$ with this new algorithm, a follow-up retrospective chart review was conducted using data from July 1, 2010-October 31, 2011 using the same inclusion and exclusion criteria as the initial review. In the follow-up assessment, patients were also excluded if the appropriate regimen for age was not selected upon vancomycin initiation. Based on this analysis, proposed adjustments have been made (Algorithm \#2) and will soon be implemented in the NCH's NICUs.

\begin{tabular}{|c|c|c|}
\hline \multicolumn{3}{|l|}{ Algorithm 1} \\
\hline $\begin{array}{c}\text { Post Menstrual } \\
\text { Age (PMA) }\end{array}$ & $\begin{array}{c}\text { Postnatal Age } \\
\text { (days) }\end{array}$ & Dosing Regimen \\
\hline$<28$ weeks & ALL & $10 \mathrm{mg} / \mathrm{kg} /$ DOSE q $12 \mathrm{hr}$ \\
\hline $28-32$ weeks & ALL & $15 \mathrm{mg} / \mathrm{kg} /$ DOSE q $12 \mathrm{hr}$ \\
\hline $33-43$ weeks & 0 to 6 & $15 \mathrm{mg} / \mathrm{kg} /$ DOSE q $12 \mathrm{hr}$ \\
& $\geq 7$ & $15 \mathrm{mg} / \mathrm{kg} /$ DOSE q $8 \mathrm{hr}$ \\
\hline$>44$ weeks & ALL & $15 \mathrm{mg} / \mathrm{kg} /$ DOSE q $6 \mathrm{hr}{ }^{* *}$ \\
\hline
\end{tabular}

**Patients $>44$ weeks on vasopressors should be $q 8 \mathrm{hr}^{* *}$

\begin{tabular}{|c|c|c|}
\hline \multicolumn{3}{|c|}{ Proposed New Dosing Algorithm \#2 } \\
\hline $\begin{array}{c}\text { Post Menstrual } \\
\text { Age (PMA) }\end{array}$ & $\begin{array}{c}\text { Postnatal Age } \\
\text { (days) }\end{array}$ & Dosing Regimen \\
\hline$<30$ weeks & ALL & $12.5 \mathrm{mg} / \mathrm{kg} /$ DOSE q $12 \mathrm{hr}$ \\
\hline $30-44$ weeks & $\begin{array}{c}0 \text { to } 6 \\
\geq 7\end{array}$ & $\begin{array}{c}15 \mathrm{mg} / \mathrm{kg} / \text { DOSE q } 12 \mathrm{hr} \\
15 \mathrm{mg} / \mathrm{kg} / \text { DOSE q } 8 \mathrm{hr}\end{array}$ \\
\hline$>44$ weeks & ALL & $15 \mathrm{mg} / \mathrm{kg} /$ DOSE q $6 \mathrm{hr}$ \\
\hline
\end{tabular}

CONCLUSIONS: Algorithm \#1 was effective at achieving goal vancomycin trough concentrations in patients $<28$ weeks and $>32$ weeks PMA. Algorithm \#2 is projected to increase the percentage of concentrations in goal range and decrease the percentage that are in the supratherapeutic range for infants $<30$ weeks PMA. There is a slight potential for higher levels in infants with a history of renal dysfunction. This group will need to be monitored closely as part of our ongoing NICU therapeutic drug monitoring quality improvement initiative.

\section{IMPLEMENTATION OF A PHARMACIST-LED PHARMACOGENOMIC SERVICE WITH RESULT RETURN AND EMR CLINICAL DECISION SUP- PORT}

Shannon Manzi, ${ }^{1}$ Vincent Fusaro, ${ }^{2}$ Catherine Clinton, ${ }^{1}$ Kenneth Mandl, ${ }^{1,2}$ David Margulies, ${ }^{1,2}$ and Catherine Brownstein ${ }^{1}$

${ }^{1}$ Boston Children's Hospital, Boston, MA, USA, ${ }^{2}$ Harvard Medical School,Boston, MA, USA shannon.manzi@childrens.harvard.edu

The Clinical Pharmacogenomics Service (CPS) was created to facilitate the incorporation of pharmacogenomic data to promote safer medication use. The service is directed by a pharmacist and governed by an Oversight Committee composed of experts who ensure the safe and rational movement of drug/gene pairs to the electronic medical record (EMR). The CPS is also responsible for the creation, maintenance and monitoring of related decision support rules in the EMR Members of the CPS include pharmacists, bioinformaticists, molecular biologists, physicians and genetic counselors. CPS pharmacists attended competency training to participate in the service. When taken alone, adverse drug reactions (ADRs) are estimated to result in 50,000 pediatric deaths in the United States annually. Genetic variability has been reported to be responsible for up to $50 \%$ of ADRs. Additionally, nearly one quarter of outpatients are prescribed drugs with genetic information in the FDA label. However, there are still important barriers that must be overcome for routine pharmacogenomic clinical use: (1) identification of clinically significant variants; (2) knowledge of variant genotype prior to prescribing; and (3) integration with EMR systems. In order to address the information gap at $\mathrm{BCH}$, the CPS elected to initially standardize thiopurine S-methyltransferase (TPMT) testing hospital-wide. The CPS officially went live with all EMR functionality in August 2012, including a pharmacogenomic results view and a consult note. In conjunction with the DNA lab, we created and implemented an interpretation report generator that converts raw data output into a clinical report. Interpretation reports are generated automatically directly from the genotype calls and then manually reviewed for accuracy. The reports and discrete haplotype results are uploaded into the EMR. Specialty flow sheet displays enable providers to easily view the results. Clinical decision support at the time of order entry by the prescriber and order verification by the pharmacist are active on all patients with an abnormal TPMT status. Alerts notify the prescriber and pharmacist that dose alteration may be required and to contact the CPS for further assistance. To date, we have run 122 samples in house with an average turnaround time of 7.5 days and a clinically actionable variant incidence of $9.7 \%$. Warfarin/ CYP2C9/VKORC1 has been identified as the next drug/gene pair to be moved into the EMR and will be implemented in the Spring of 2013. Additional drug/gene pairs and exome regions of interest (ROIs) are being evaluated by the CPS Oversight Committee and will moved into the EMR in the near future. As pre-emptive genomic sequencing becomes routine, standardized methods to are needed to interpret data and facilitate clinical decisions in real time. In conjunction with BCH DNA Laboratory, we streamlined the TPMT testing process, including ordering, testing, and return of results and developed a pharmacist-led consultative service.

THE ROLE OF EMERGENCY MEDICINE PHARMACY PRACTICE IN A CHILDREN'S HOSPITAL Jenny Mason and Matthew Sapko 
Nationwide Children's Hospital, Columbus, OH, USA jenny.mason@nationwidechildrens.org

BACKGROUND: The emergency department (ED) is an integral practice environment for pharmacists because of high-risk patients, urgent need for medications, use of more high-alert medications, and limited prospective review by pharmacists prior to medication administration. Within this setting medication errors are common especially in pediatric patients. The implementation of an emergency medicine pharmacist (EMP) in a pediatric ED could greatly reduce medication errors, improve overall care, and enhance clinical pharmacy services.

The American Society of Health-System Pharmacists has identified both essential and desirable services that an EMP can provide. Essential services include direct patient care, medication order review, medication therapy monitoring, drug information, and qualityimprovement initiatives. Medication reconciliation, education, and research are services that are desirable to optimize outcomes. In September 2012 two EMPs were integrated into practice at Nationwide Children's Hospital. The objective of implementing EMPs was to justify a clinical staffing position that can provide clinical pharmacy services that focus on preventing errors, improving medication therapy and patient outcomes, administrative and quality-improvement projects, and providing distributive pharmacy functions. METHODS: An EMP provides coverage within the ED for 10 hours a day, seven days a week during peak ED hours. Two EMPs began a 6-week training program in August 2012. Training included both staffing and clinical components as well as brainstorming sessions for the essential and desirable services that would be provided. Direct patient care activities included the EMP at bedside for high-alert medications and procedures such as traumas, cardiac arrests, seizures, and intubations. Medication order review and drug information were also top priorities. As the practice model evolved other services such as medication therapy management with antibiotic optimization for complex patients and medication procurement and preparation developed. Involvement in administrative functions including quality-improvement initiatives and education were a core expectation and included in the staffing structure. More recently the EMPs have been involved with training and implementing pharmacy students to obtain medication reconciliation for admitted and complex patients. RESULTS: The EMP provides documentation for all clinical interventions. The majority of interventions during the first six months include direct patient care at bedside for critical patients, antibiotic evaluation, therapeutic drug monitoring, and drug information. Medication reconciliation has been increasing with implementation of the pharmacy students. Involvement in quality-improvement initia- tives include projects related to sickle cell, febrile neutropenia, and antibiotic therapy for intubated patients, a nurse-initiated steroid administration protocol, and review of ED order sets. CONCLUSION: Implementation of the EMPs have been well received within the emergency and pharmacy departments. This has provided more collaboration between each department and enhanced the transition of care. Areas of future growth may include automated dispensing system profiles, culture review, and additional quality-improvement initiatives and education opportunities.

\section{JAMAICAN A DIFFERENCE: A PEDIATRIC PHAR- MACY MISSION TRIP}

Kelly Matson and Courtney Barnas

University of Rhode Island, Kingston, Rhode Island, USA matson@uri.edu

Pharmacist involvement as part of medical mission teams has been increasing through natural disaster relief efforts. Pharmacists have the ability to be involved in mission efforts on many levels, including formulary and drug resource development, medication and disease state education, drug dosing and calculations, physical assessment and its education of patients and caregivers. Given the increasing need of pharmacists within missions, it was identified to begin the development of a pediatric pharmacy student-led mission trip to Jamaica, which would include 11 pharmacy students and a pediatric faculty member. As part of establishing a pharmacy mission, our goals were to enhance the students' practice-based abilities, including teamwork, communication and leadership skills, professionalism and the importance service as a healthcare professional. To accomplish our practice-based goals, as well as, the ultimate goal of improving medication safety and effectiveness for Jamaican children within the Mustard Seed Communities (MSC), we developed educational workshops on proper medication use.

Our journey to establish a pediatric pharmacy mission began with identifying support from a community organization. Mustard Seed Communities is a non-profit organization that cares for over 600 children in 4 countries who have been abandoned, who have mental and physical disabilities or who are affected by HIV or AIDS. Our pharmacy-student leader had traveled with the organization previously and approached the MSC executive director to begin the discussion of a pharmacy mission. Once established, we began the planning stages of determining the medication use need in the 10 Jamaican communities MSC serves. We developed workshop materials and interactive activities for the caregivers of MSC communities to provide education on the following: proper medication use of otic, optic, nasal, rectal and oral medications; basic pediatric physical assessment skills; importance of accurate record keeping of medication administra- 
tion, as well as, adherence to dosing regimens; and HIV treatment and medications. Additionally, 10 drug information resource books for each community were developed to complement the MSC formulary, highlighting medication use, pediatric dosing, interactions, warnings, and side effects and educated upon during workshops.

Travel to Jamaica was one week during winter break. Upon returning, pharmacy students voluntarily participated in a 5-point Likert-scaled survey focusing on practice-based abilities. All student survey results were positive in the 20-item practice-based abilities. Students felt better prepared to work as a team, communicate healthcare knowledge and medication use to pediatric patients and caregivers, and perform physical assessment and pediatric dosing calculations. They felt the mission trip enhanced their leadership skills, their role in service activities and their ability to provide pediatric patient care. The pharmacy mission team also received accolades from the MSC directors for a successful trip and great appreciation for the education and resources shared.

\section{DOES COMBINATION THERAPY WITH VAN- COMYCIN AND PIPERACILLIN-TAZOBACTAM INCREASE THE RISK OF NEPHROTOXICITY IN PEDIATRIC PATIENTS?}

Katie McQueen, Dana Clark, and Jennifer SternerAllison

Children's Healthcare of Atlanta, Atlanta, Georgia, USA katie.mcqueen@choa.org

INTRODUCTION: Vancomycin and piperacillintazobactam (PIP/TAZ) are often used concomitantly for broad spectrum antibacterial coverage. Recent publications have estimated that as many as 50\% of patients treated with the combination of vancomycin and PIP/TAZ develop acute kidney injury. Data to support this finding is minimal and there is almost no data in the pediatric population. This investigation aimed to determine if pediatric patients treated with vancomycin and PIP/TAZ had a higher incidence of nephrotoxicity than those treated with vancomycin alone. METHODS: A retrospective chart review of patients treated with vancomycin or vancomycin in conjuction with PIP/TAZ between November 1, 2012 and January 16, 2013 was conducted. The population included patients $\leq 19$ years of age who were treated at both campuses of Children's Healthcare of Atlanta. Patients had received either vancomycin for $\geq 48$ hours or vancomycin and PIP/TAZ for $\geq 48$ hours simultaneously. Data collected included patient demographics, baseline and repeat BUN and serum creatinine values, vancomycin troughs, vancomycin dose and interval, PIP/TAZ dose and interval, other nephrotoxic agents administered (amphotericin, aminoglycosides, ketorolac, and furosemide), and intensive care unit (ICU) stays. Serum creatinine and BUN values were trended to determine if patients had nephrotoxicity, which was defined as a $100 \%$ increase or greater in baseline serum creatinine or an increase of at least $0.5 \mathrm{mg} / \mathrm{dL}$ from the baseline serum creatinine. The secondary outcomes were to determine if ICU stays, higher vancomycin troughs $(>15 \mathrm{mg} / \mathrm{mL})$, or receiving other nephrotoxic agents were related to developing nephrotoxicity. Percentages of patients who developed nephrotoxicity in the vancomycin group and the combination therapy group were calculated and compared. RESULTS: During the study period there were 16 patients in the vancomycin group and 106 patients in the vancomycin with PIP/TAZ group. Of the 16 patients in the vancomycin alone group, one patient developed nephrotoxicity $(0.0625 \%)$. This patient had a vancomycin trough $\leq 15 \mathrm{mg} / \mathrm{mL}$, was in the ICU, and received other nephrotoxic agents. In the vancomycin plus PIP/TAZ group, 25 of the 106 patients $(23.6 \%)$ developed nephrotoxicity. Furthermore, $78 \%$ of the patients with nephrotoxicity had vancomycin troughs $>15 \mathrm{mcg} / \mathrm{ml}$, while $80 \%$ had an ICU stay and $76 \%$ received other nephrotoxic medications. CONCLUSIONS: Patients treated with vancomycin in conjunction with PIP/TAZ are at higher risk for nephrotoxicity; however, the higher incidence cannot be solely attributed to the combination alone. As evidenced by the secondary outcomes, ICU stays, higher vancomycin troughs, and concomitant use of other nephrotoxic agents cannot be excluded as risk factors for nephrotoxicity. Increased monitoring of renal function and vancomycin troughs is warranted in these patients.

\section{EVALUATION OF ANTIBIOTIC USE IN PEDIAT- RIC URINARY TRACT INFECTIONS}

Rachel Meyers, ${ }^{1,2}$ Jessica Ohiri, ${ }^{1}$ Ajay Shukla, ${ }^{1}$ and Falguni Shah ${ }^{2}$

${ }^{1}$ Ernest Mario School of Pharmacy, Rutgers University, Piscataway, NJ, USA, ${ }^{2}$ Saint Barnabas Medical Center, Livingston, NJ,USA rachel.meyers@pharmacy.rutgers.edu

INTRODUCTION: The current pediatric urinary tract infection (UTI) guidelines indicate that the choice of antibiotic to treat UTIs should be based on local antimicrobial sensitivity patterns. Escherichia Coli (E. Coli) is the most common pathogen in UTIs. This institution, which includes a 30 bed general pediatric unit and a 10 bed pediatric intensive care unit within an adult hospital, has seen sensitivities of E.Coli to the antibiotic cefazolin which consistently exceed $80 \%$. Despite this, the agent chosen to treat UTIs is usually ceftriaxone. We undertook an investigation of pediatric patients admitted to our hospital with UTIs to determine if there are any risk factors for resistance to cefazolin and to determine if antibiotic therapy is being appropriately de-escalated once sensitivities are known. METHODS: Medical charts were retrospectively evaluated for patients 21 years and younger 
who were treated for a urinary tract infection or pyelonephritis in the inpatient setting between January 2010 and December 2011. Data collected included age, weight, history of present illness, urinalysis results, urine culture and sensitivity data, and the antibiotics used to treat the infection. RESULTS: Data was collected for 99 patients. Fifty percent of urine cultures had no growth, $27 \%$ grew E. Coli, $15 \%$ grew Enterococcus faecium, and $8 \%$ of cultures grew other pathogens. Of the 51 E.Coli isolates, $94 \%$ were sensitive to cefazolin. Of the 5 patients who grew an E.Coli that was resistant to cefazolin, 4 had a significant past medical history that included urinary tract abnormalities. The initial antibiotic prescribed was ceftriaxone in $42 \%$ of cases. Fifteen percent of patients were neonatal rule-out sepsis cases who received ampicillin and cefotaxime, $12 \%$ of patients received cefepime, $5 \%$ of patients received levofloxacin, and $26 \%$ of patients received some other antibiotic. Of patients who grew E. Coli, $86 \%$ grew a strain sensitive to cefazolin but were continued on ceftriaxone. Only 1 patient $(5 \%)$ had an E. Coli that was sensitive to cefazolin and had antibiotic therapy switched to the oral equivalent, cephalexin. CONCLUSIONS: At our hospital, the sensitivity of E. Coli strains from the urine to cefazolin warrants the change of first line therapy from ceftriaxone to cefazolin. Pediatricians should screen patients for past medical history to determine if they are at higher risk for a resistant strain. We plan to initiate an education program for our pediatricians to address this issue.

\section{ETHACRYNIC ACID CONTINUOUS INFUSIONS IN CRITICALLY-ILL PEDIATRIC PATIENTS}

Jamie Miller, Jared Schaefer, Matthew Tam, Donald Harrison, and Peter Johnson

University of Oklahoma College of Pharmacy, Oklahoma City, OK, USA jamie-miller@ouhsc.edu

INTRODUCTION: Loop diuretic continuous infusions (CI) are often used in ICU's to manage oliguria and volume overload in hemodynamically unstable, critically-ill children. Furosemide is most commonly used; however, current shortages have increased the demand for alternatives such as ethacrynic acid. The purpose of this study was to describe the dosage regimen and treatment outcomes in critically-ill children receiving ethacrynic acid CI. METHODS: This retrospective cross-sectional study evaluated patients less than 18 years of age who received an ethacrynic acid $\mathrm{CI}$ exceeding 12 hours between the dates of January 1, 2007 through January 31, 2012. The primary objective of this study was to determine the mean and median dose of ethacrynic acid CI. Secondary objectives were to assess the effectiveness of the therapy using markers [e.g., urine output (UOP), fluid balance] and the number of patients with altered serum electrolytes (i.e., potassium, sodium, chloride, and mag- nesium) or metabolic alkalosis, requiring intervention. Descriptive statistics were used to evaluate the results. A series of repeated-measures analyses of variance (ANOVA) were conducted to assess differences in the surrogate markers of efficacy (i.e., UOP, fluid balance, glomerular filtration rate [GFR]) and adverse events that occurred pre-, mid-, and post-ethacrynic acid CI. RESULTS: Nine patients were included for analysis. The mean initial and maximum doses $(\mathrm{mg} / \mathrm{kg} /$ hr) were $0.13 \pm 0.07$ (median 0.1, range 0.08-0.3) and $0.17 \pm 0.08$ (median 0.16 , range $0.09-0.3$ ), respectively. The median UOP $(\mathrm{mL} / \mathrm{kg} / \mathrm{hr}$ ) pre-, mid-, and postinfusion (interquartile range [IQR]) was 2.4 (1.8-3.2), 4.2 (3.5-6), and 4 (3.4-5.3), respectively. The median fluid balance (mL) (IQR) was 189 (90 to 526), -258 (-411.7 to 249 ) and -113.5 (-212.5 to 80.2$)$, respectively. There were statistically significant differences in UOP and fluid balance pre- versus mid-therapy $(0.014)$, as well as pre- versus post-therapy $(\mathrm{p}=0.01)$. No significant differences were noted with GFR, magnesium, sodium, chloride, and potassium concentrations. Five children (55.6\%) were treated for metabolic alkalosis. CONCLUSION: This study provides preliminary evidence for ethacrynic acid CI in children. The median initial dose and maximum dose in this cohort were $0.13 \mathrm{mg} / \mathrm{kg} /$ hour and $0.17 \mathrm{mg} / \mathrm{kg} /$ hour, respectively. Larger prospective studies are needed to confirm these findings.

\section{OPIOID USE AND THE RISK OF RESPIRATORY DEPRESSION AND DEATH IN THE PEDIATRIC POPULATION}

Marianne Miscioscia

New Hampshire's Hospital for Children at Elliot Health System, Manchester, NH, USA mmisciosc@elliot-hs.org

BACKGROUND: Pediatric patients are at an increased risk of adverse effects from various medications. Recently, there have been a number of serious cases, including several pediatric patients suffering from severe respiratory depression and death as a result of the use of codeine for pain control following tonsillectomy and adenoidectomy. OBJECTIVE: To assess the safety of codeine and other opioid agonists in all pediatric patients and evaluate the risk of respiratory depression and death. METHODS: Pubmed and Medline were used to obtain randomized controlled studies from 2002 to 2012. Relative risks and confidence intervals were calculated using data available in clinical trials. RESULTS: A total of eleven clinical trials were evaluated for this review. Randomized controlled trials included studies comparing opioids versus non-opioids for a variety of painful conditions. The relative risk of respiratory depression associated with opioid use in one trial was 1.63 (95\% confidence interval (CI): 0.64-6.13). The remaining ten trials reviewed resulted in no significant difference in respiratory 
depression, or adverse effects associated with treatment identified in the study patients. No deaths were attributed to opioid use in any of these studies. CONCLUSION: Respiratory depression and death were not common adverse effects in the studies reviewed, thus the population evaluated were not at an increased risk of serious events following the administration of opioids. Additional randomized controlled trials specifically investigating respiratory depression are further warranted.

\section{VANCOMYCIN LOADING DOSE FOR RAPID ACHIEVEMENT OF TARGET TROUGH CONCEN- TRATIONS IN CHILDREN}

AlJohara Al-Sakran, ${ }^{1,3}$ Jill A. Morgan, ${ }^{1}$ and Kristine A. Parbuoni ${ }^{2}$

${ }^{1}$ University of Maryland School of Pharmacy, Baltimore, MD, USA, ${ }^{2}$ University of Maryland Medical System, Baltimore, MD, USA, ${ }^{3}$ King Faisal Specialist Hospital, Ar Rawdah, Jeddah 23433, Saudi Arabia jmorgan@ rx.umaryland.edu

OBJECTIVE: To determine the impact of a vancomycin loading dose of $25-30 \mathrm{mg} / \mathrm{kg}$ on rapid achievement of target therapeutic serum trough concentrations (15-20 mg/L).

METHOD: A retrospective study was conducted in the pediatric intensive care unit (ICU). Patients were included if their age was 1 month to 18 years, received vancomycin at a dose of $60 \mathrm{mg} / \mathrm{kg} /$ day, had normal renal function, and levels were drawn appropriately. The control group included patients who did not receive a loading dose and the treatment group included patients who received a loading dose. All initial vancomycin trough concentrations were examined. The primary outcome measure was the proportion of patients who achieved target trough concentrations (15-20 $\mathrm{mg} / \mathrm{L}$ ) as a first concentration obtained within 48 hours of vancomycin initiation while the secondary outcome measure was the mean time to reach a target trough. RESULTS: The number of vancomycin patients that met the inclusion criteria was 57 , with 49 patients in the control group and 8 patients in the loading dose group. The proportion of patients that achieved a target therapeutic trough in the control group was $5 / 49(10.2 \%)$ versus two out of eight $(25 \%)$ in the loading dose group, $p=0.25$. The mean time to reach a target trough could not be determined due to inconsistency in practice with regard to obtaining a second trough after the dose was increased. A total of 28 courses in group A (57\%) and five courses $(62 \%)$ in group B never reached the target trough. DISCUSSION: In this study, a vancomycin loading dose did not result in rapid attainment of target trough concentrations $(15-20 \mathrm{mg} / \mathrm{mL})$, however; the small sample size was a major limitation and may have impacted the result. The study however; demonstrated inadequacy of the $60 \mathrm{mg} / \mathrm{kg} /$ day dose. Studies with larger sample sizes are needed to further evaluate the efficacy and safety of this intervention in pediatric patients.

\section{STANDARDIZATION OF EXTEMPORANEOUS ORAL PRODUCTS BETWEEN UNIVERSITY OF KENTUCKY MEDICAL CENTER AND KENTUCKY CLINIC PHARMACY - PREVENTION OF MEDICA- TION ERRORS \\ Allison Mruk, Lisa Brantley, and Brian Gardner University of Kentucky, Lexington, KY, USA allison. mruk@gmail.com}

INTRODUCTION: One-third of adverse drug events are associated with medication errors and considered preventable. The risk for pediatric medication errors is high, with the most common being prescriptionrelated errors. For premature neonates, transplant patients, and patients with complicated medication regimens, Kentucky Children's Hospital $(\mathrm{KCH})$ ensures that parents can accurately administer oral medications prior to hospital discharge. Physicians place a "Care by Parent" order allowing parents the opportunity to learn how to withdraw the correct doses of oral suspensions/solutions under staff supervision. Many discharge prescriptions from $\mathrm{KCH}$ are filled by the Kentucky Clinic Pharmacy (KCP) located on the Medical Center campus. Therefore, it is imperative that all oral medications administered via "Care by Parent" are the same concentration that is used at KCP. Due to a recent medication error, this project was initiated to standardize all oral medication concentrations. OBJECTIVES: The primary objective was to identify differing concentrations and standardize all extemporaneous oral products between $\mathrm{KCH}$ and $\mathrm{KCP}$. The secondary objectives were to assure all extemporaneous oral product recipes were supported by published evidence, and establish a single concentration in CPOE to prevent additional possible errors. METHODS: Separate databases of inpatient and outpatient compounded oral products were reviewed and oral medication concentrations that differed between $\mathrm{KCH}$ and $\mathrm{KCP}$ were identified. A search including PubMed, ASHP's "Extemporaneous Formulations for Pediatric, Geriatric, and Special Needs Patients" Pediatric Dosage Handbook, Paddock Laboratories and Perrigo websites, was conducted to assure all extemporaneous oral products had referenced evidence. For medications that had multiple concentrations available or recipes not referenced, the pharmacy enterprise committee met to determine which single referenced recipe should be used. A standard template was created to allow for inclusion of complete production instructions and documentation of all extemporaneous oral products at both sites. Every referenced concentration was confirmed and appropriately adjusted in CPOE. RESULTS: Ninety-six recipes were reviewed. Eight oral medications had differing concentrations 
between $\mathrm{KCH}$ and $\mathrm{KCP}, 13$ had multiple concentrations available in the system, and 11 recipes were not referenced. For each of these 32 recipes, the committee selected one referenced concentration that was most appropriate for all patients. CONCLUSIONS: Prescription-related errors are the most common type of medication error in pediatric patients. Eight of the 96 recipes reviewed had a different concentration between $\mathrm{KCH}$ and $\mathrm{KCP}$ and could have led to potential error. An additional 13 had multiple concentrations which could have led to potential dosage errors. As a result of this project, $100 \%$ of compounded suspensions are now consistent between $\mathrm{KCH}$ and $\mathrm{KCP}$ and contain referenced data assuring valid production and stability.

\section{SUCCESSFUL ORAL LINEZOLID DESENSITIZA- TION IN A CYSTIC FIBROSIS PATIENT WITH MULTIPLE DRUG ALLERGIES}

Kimberly Novak, ${ }^{1,2}$ Kristen Gawronski, ${ }^{1,3}$ and Stephen Kirkby $^{1,3}$

${ }^{1}$ Nationwide Children's Hospital, Columbus, OH, USA, ${ }^{2}$ The Ohio State University College of Pharmacy, Columbus, OH, USA, ${ }^{3}$ The Ohio State University Wexner Medical Center, Columbus, OH, USA kimberly.novak@ nationwidechildrens.org

BACKGROUND: A 24-year old female with a history of cystic fibrosis (CF) was admitted to a free-standing children's hospital for treatment of a CF pulmonary exacerbation. CF sputum culture history was notable for methicillin-resistant Staphylococcus aureus (MRSA) and methicillin-sensitive Staphylococcus aureus (MSSA). She had previously failed outpatient treatment with minocycline and had a history of significant allergic reactions to both vancomycin and linezolid (lip swelling and itching). The patient was initiated on IV quinupristin/dalfopristin; however, issues with IV access necessitated reevaluation of alternative antibiotic options. METHODS: An oral linezolid desensitization protocol was designed and implemented using a commerciallyavailable suspension. The protocol involved an eight-dose hourly escalation scheme starting with an initial dose of $1 / 80,000$ of the target dose $(600 \mathrm{mg})$ and increasing to $1 / 8,000,1 / 800,1 / 80,1 / 8,1 / 4,1 / 2$, and final target doses respectively. The patient was additionally premedicated with diphenhydramine $25 \mathrm{mg}$ and ranitidine $150 \mathrm{mg}$ by mouth; both were continued at twice daily dosing throughout the antibiotic course. RESULTS: The patient completed successful linezolid desensitization and was discharged home after demonstrating 48 hours of inpatient antibiotic tolerance to complete 14 days of linezolid therapy. Subsequently, the patient has tolerated multiple linezolid desensitizations for her CF pulmonary exacerbations using the same protocol, with tolerance of linezolid courses as long as 30 days. CONCLUSION: This is the first case report describing a successful desensitization protocol using oral linezolid suspension in a CF patient with multiple drug allergies.

PEDIATRIC PHARMACY TECHNICIANS IMPROVING SAFETY AND CHILDREN'S ACCESS TO CARE

Ozioma Ogbuokiri, Cindi Haley, Nicole Work, and Priya Patel

Cook Children's Healthcare System, Fort Worth, TX, USA ozioma.ogbuokiri@cookchildrens.org

BACKGROUND: Pharmacy department leadership held a strategic planning retreat in 2011 and set forth goals to elevate and promote the role of the pharmacy technician. We determined there was a need for dedicated staff responsible for data collection and analysis related to medication safety and business management. We also realized we were dispensing increasing amounts of medication at the expense of the institution to patients without taking advantage of programs made available by pharmaceutical manufacturers that assist patients with cost and/or reimburse the hospital for the cost of the drug. This was costing the hospital approximately $\$ 30,000$ annually in medication expense that was written off. This cost is steadily increasing due to $22 \%$ of our county's population being uninsured. RESULTS: We justified and received administrative approval for three positions-medication safety technician, data technician, and patient assistance program technician. The medication safety technician supports the pharmacy department and medication safety officer in collecting data, creating quality and operational dashboards, defining metrics and reviewing and referring medication-related event reports and near misses. The medication safety technician assists with implementation of safety initiatives in the hospital. Examples of safety initiatives include decreasing the number of sterile products made without barcode scanning by $50 \%$, standardizing the method of reviewing event reports and near misses, and various gap analyses on our processes. The data technician supports the business manager in overseeing four cost centers with an annual budget of $\$ 185,000,000$ through recovering revenue, reviewing billing discrepancies, and analyzing data to ensure optimal use of technology and financial resources. Since beginning the position in January 2012, the data technician has resolved 16,612 billing discrepancies each resulting in recovered revenue of up to $\$ 130,000$ per patient course. The patient assistance program technician coordinates patients needing medication due to lack of financial resources. The patient assistance program technician works with families, social workers, case managers, and pharmaceutical companies to ensure the organization is dispensing medications to only the patients with demonstrated need and the hospital recovers as much of the expense as possible. In the first twelve months of the program, the patient assistance program technician has helped 653 families get the medications they need using $\$ 61,693$ in chari- 
table care, with $\$ 21,476$ reimbursed for those services. CONCLUSION: Growing the role of the pharmacy technician has been a positive experience that has improved employee job satisfaction, patient safety, reduced cost, and improved efficiency. There are plans underway to implement clinical pharmacy technicians to support the clinical pharmacists on the patient care units. As institutions are challenged with providing optimal services and managing costs, incorporating pharmacy technicians is vital to achieving organizational goals.

RETROSPECTIVE EVALUATION OF ANTITHROMBIN III SUPPLEMENTATION IN NEONATES AND INFANTS RECEIVING ENOXAPARIN FOR TREATMENT OF THROMBOSIS

Alexandra Oschman, ${ }^{1}$ Amy Corder, ${ }^{2}$ Damon Pabst, ${ }^{1}$ and Kristin Held ${ }^{1}$

${ }^{1}$ Children's Mercy Hospital and Clinics, Kansas City, Missouri, USA, ${ }^{2}$ TriStar Centennial Medical Center, Nashoille, Tennessee,USA alex@oschman.com

INTRODUCTION /OBJECTIVE: During treatment with enoxaparin, neonates and infants at Children's Mercy Hospital \& Clinics (CMHC) are often supplemented with antithrombin III (AT3) to replace physiologically low AT3 levels. The proposed rationale behind AT3 supplementation is augmentation of enoxaparin activity to decrease time to achievement of a therapeutic anti-Xa level. Literature has not been published on this practice. METHODS/DESIGN: Institutional review board approval was obtained for this single-centered, retrospective study. Patients less than 1 year of age treated with enoxaparin for thrombus were included with (Group 1) and without (Group 2) AT3 supplementation from July 2006 through July 2011. Exclusion criteria included: concurrent anticoagulation other than enoxaparin or AT3, predisposition for bleeding or thrombus formation, pre-existing renal dysfunction, uncollected or inappropriately collected anti-Xa levels, or patients receiving enoxaparin at admission. The primary objective was to compare time to therapeutic anti-Xa levels between the two groups. Secondary objectives were to characterize enoxaparin dosing and dose changes to achieve therapeutic anti-Xa levels along with bleeding events and cost associated with AT3 supplementation. Critical illness, age, and timing of AT3 initiation were assessed as potential confounding variables. Equivalence between primary and secondary endpoints were evaluated utilizing Schuirmann's two one-sided test. Statistical equivalence was defined as a $p<0.05$. Logistic regression and a generalized linear model procedure were performed to assess independent effects of critical illness, age, or timing of AT3 initiation on time to therapeutic anti-Xa levels. RESULTS: Sixty patients met inclusion and exclusion criteria (group $1=34$ patients and group $2=26$ patients); demographics were similar between groups with the exception of a higher platelet count in group $2(p=0.01)$. Mean time to therapeutic anti-Xa level was similar between groups 1 and 2 (80.7 hours versus 65.2 hours; $\mathrm{p}=0.55)$. Dosing required to attain a therapeutic level was not equivalent between groups $(p=0.18)$, while number of dose changes were equivalent ( $p=0.0002)$. Bleeding events between groups were not equivalent. Five patients (14.3\%) in group 1 and 1 patient $(3.9 \%)$ in group 2 experienced bleeding resulting in discontinuation/dose reduction of enoxaparin $(p=0.55)$. Critical illness, age, and timing of AT3 did not appear to affect time to therapeutic anti-Xa levels, number of dose changes, dose to achieve a therapeutic anti-Xa level, or incidence of bleeding. Patients less than 2 months received higher initial doses $(1.48 \mathrm{mg} / \mathrm{kg}$ subcutaneously every 12 hours versus $1.12 \mathrm{mg} / \mathrm{kg}$ subcutaneously every 12 hours, $\mathrm{p}<0.001)$. Over 5 years approximately $\$ 1.2$ million was spent on 750 doses of AT3. CONCLUSIONS: Supplementation did not decrease time to therapeutic anti-Xa levels and was associated with increased risk of bleeding. In addition, supplementation added a substantial cost to the institution without a detectable benefit.

\section{THE USE OF INTRAVENOUS (IV) ACETAMINO- PHEN IN A NEONATAL INTENSIVE CARE UNIT (NICU) \\ Lindsay Patrick and James Dice \\ Children's Hospital of the King's Daughters, Norfolk, VA, USA lindsay.patrick@chkd.org}

BACKGROUND: The use of IV acetaminophen in neonatal intensive care units in the United States has been increasing. U.S studies have been limited; therefore FDA approval for patients less than 2 years of age has not been established. Varying neonatal dosing guidelines have been recommended for patients 28 weeks corrected gestational age to $\geq 37$ weeks corrected age. Many patients return from surgery unable to take medications enterally. Rectal acetaminophen has erratic and variable absorption which makes proper dosing difficult and pain management suboptimal. We began using IV acetaminophen post-operatively at Children's Hospital of the King's Daughters (CHKD) in the NICU for patients $\geq 37$ weeks corrected age. The standard dose of acetaminophen used is $10 \mathrm{mg} / \mathrm{kg} /$ dose every 6 hours for a duration of 48 hours with a maximum daily dose of $40 \mathrm{mg} / \mathrm{kg} /$ day. The purpose of this review is to report our experience using IV acetaminophen in infants $\geq 37$ weeks corrected age. Methods: We performed a retrospective review of patient's medical records. All patients admitted to the NICU who received IV acetaminophen were eligible for study inclusion. All patients were 37 weeks corrected age or older. Most patients were also receiving morphine or fentanyl infusions in addition to IV acetaminophen. Data collection included: demographic data, 
surgical procedure performed, infusion rate of opioids, number of "as needed" doses daily, and Neonatal Pain, Agitation, and Sedation Scale (N-PASS) scores. RESULTS: 19 patients received IV acetaminophen in the NICU. 13 patients were Caucasian. The gestational age ranged from 23-39 weeks. The weight of the patients ranged from 1.6 to 6.7 kilograms. The average infusion rate of morphine when IV acetaminophen was started was $35 \mathrm{mcg} / \mathrm{kg} / \mathrm{hr}$. The average infusion rate of morphine when IV acetaminophen was discontinued was $37 \mathrm{mcg} / \mathrm{kg} / \mathrm{hr}$. The average number of "as needed" morphine doses given was 3 . The average fentanyl infusion rate when the IV acetaminophen was started and discontinued was $2.6 \mathrm{mcg} / \mathrm{kg} / \mathrm{hr}$. The N-PASS pain scores ranged from 0-6 for the first 48 hours post-op while receiving IV acetaminophen. CONCLUSION: Upon review of our IV acetaminophen use in post-operative NICU patients, it appears that IV acetaminophen is both safe and effective. IV acetaminophen has helped to aid in pain management when given post-operatively to patients in our NICU. IV acetaminophen use and adverse effects will continue to be monitored.

OUTCOMES ASSESSMENT OF AN ANTIMICROBIAL STEWARDSHIP PROGRAM ON VANCOMYCIN PRESCRIBING PRACTICES IN PEDIATRIC INTENSIVE CARE UNITS

Emily Polischuk, ${ }^{1,2}$ Kelli L. Crowley, ${ }^{1,2}$ Carol G. Vetterly, ${ }^{1,2}$ James E. Levin, ${ }^{3}$ and Michael Green ${ }^{3}$

${ }^{1}$ Children's Hospital of Pittsburgh of UPMC, Pittsburgh, PA, USA, ${ }^{2}$ University of Pittsburgh School of Pharmacy, Pittsburgh, PA, USA, ${ }^{3}$ Children's Hospital of Pittsburgh of UPMC Division of Infectious Diseases, Pittsburgh, PA, USA emily.polischuk@chp.edu

OBJECTIVE: In April 2010, Children's Hospital of Pittsburgh of UPMC (CHP) expanded its Antimicrobial Stewardship Program (ASP) to include vancomycin. The ASP team, unit-based clinical pharmacists and infectious disease physicians, aims to optimize clinical outcomes while minimizing unintended consequences of antimicrobial use. Vancomycin usage algorithms were developed with key services prior to commencement. At 72 hours of vancomycin therapy, ASP provides a Day 3 Vancomycin Note in the medical record containing recommendations on continued use. The purpose of this retrospective chart analysis was to describe the impact of ASP on vancomycin use in the pediatric, neonatal and cardiac critical care units over time. METHODS: CHP's Clinical Data Warehouse was used to generate vancomycin usage reports in the critical care units. Reports contained data from the first quarter of 2008 through the third quarter of 2012. The data collected included the percentage of total vancomycin days per total patient days, the percentage of vancomycin cases per total patient cases and overall total doses dispensed. The total milligrams (mg) of vancomycin dispensed were used to detect a possible cost savings. Day 3 Vancomycin Notes were reviewed for the acceptance of recommendations starting January 2011 (when Data Warehouse report developed). RESULTS: Since April 2010, the \% vancomycin days / total patient days in the intensive care units has decreased from $25.2 \%$ to $12.6 \%$, a decrease of $50 \%$. In the two years prior to the program, the median quarterly \% vancomycin days/total patient days was $28.2 \%$; this has decreased to a median of $20.6 \%$ over time since surveillance began. A similar reduction was observed in vancomycin initiations, with a $42 \%$ decrease in the cardiac intensive care unit, a $32 \%$ decrease in the pediatric intensive care unit, and a $57 \%$ decrease in the neonatal intensive care unit. The total doses dispensed decreased by $45 \%, 44 \%$, and $40 \%$ in the cardiac, pediatric, and neonatal intensive care units, respectively. The average monthly spending on vancomycin in the year prior to surveillance was $\$ 2,350$; this decreased to a monthly average of $\$ 1576$ in the last quarter of data collection. All Vancomycin Day 3 Notes $(n=433)$ were reviewed, with an overall acceptance rate of $76 \%$. The acceptance rate in 2012 was $78.5 \%$, which was a $4 \%$ increase from the previous year. CONCLUSION: This data demonstrates that the ASP at the Children's Hospital of Pittsburgh of UPMC has likely contributed to a large reduction in the use of vancomycin in the intensive care units. The majority of recommendations made by ASP members are accepted by the medical team.

\section{A RETROSPECTIVE EVALUATION OF OPIOID WEANING USING METHADONE IN OPIOID- DEPENDENT PEDIATRIC POPULATIONS}

Kazim Giby, Régis Vaillancourt, Christina Vadeboncoeur, Nisha Varughese, Annie Pouliot, and Carmen Ma Children's Hospital of Eastern Ontario, Ottawa, ON, Canada apouliot@cheo.on.ca

RATIONALE: Critically ill children in pediatric care are routinely treated with opioids to alleviate pain and anxiety, increase co-operation, and reduce metabolic demands. However, prolonged exposure to short halflife opioids followed by an abrupt cessation frequently leads to opioid withdrawal syndrome (OWS). Opioid weaning protocols aim to gradually decrease opioid plasma concentration to prevent OWS symptoms. OBJECTIVE: To evaluate the non-protocolized use of methadone to prevent opioid withdrawal syndrome in pediatric population. METHOD: A retrospective chart review of thirty children (0-18 years) who were successfully weaned off intravenous morphine, fentanyl, or hydromorphone infusions (all standardized to equivalents of intravenous morphine) using enteral methadone was performed at a tertiary care pediatric teaching hospital from 2006 to 2012. RESULTS: Qualitative analysis of non-protocolized 
use of methadone for opioid withdrawal symptoms revealed four distinct phases of methadone dosing which were not discussed in previous literature: an opioid overlap phase, a loading dose phase, a dose maintenance phase, and a linear tapering phase. Twenty-seven of 30 patients (90\%) showed at least three of the four phases in their dosing schedules. Patients were divided into short duration, medium duration, and long duration methadone weaning groups based on the duration of the tapering phase. Regression analysis of daily methadone doses (measured as \% of max daily methadone dose) in each weaning group showed significant differences in daily dose reduction during the tapering phase $(\mathrm{p}=0.01)$, and all weaning groups demonstrated linear and predictable daily dose reductions ( $R 2 \geq 0.7$ ). Twenty-nine of 30 patients $(96.7 \%)$ were able to safely take at least one concomitant sedative medication that affects opioid/ methadone dosing during the wean. The average number of concomitant sedatives taken during methadone weaning was $4.63 \pm 2.09$. The majority of patients $(66.7 \%)$ were mechanically ventilated during the opioid/methadone weaning process. Of the patients who received methadone while intubated $(33.3 \%$ of all patients), the number of days intubated while on methadone was strongly correlated to the duration of the opioid overlap phase and the duration of the dose maintenance phase $(\mathrm{p}=0.01)$. Overall, intubated patients received significantly higher doses of methadone during the opioid overlap phase $(0.664 \mathrm{mg} / \mathrm{kg} /$ day compared to $0.397 \mathrm{mg} / \mathrm{kg} /$ day) and also had higher opioid doses on the day before weaning $(p=0.05)$. CONCLUSIONS: Retrospective analysis of the non-protocolized methadone weans at this institution reveals similarities in patterns on the level of methadone dosing schedules in the form of four phases (overlap, loading, maintenance, and tapering). While non-protocolized methadone weans were highly variable in duration and dosage, this study showed the four phase structure may be applied to patients with complicating factors, such as mechanical ventilation, who are not suitable for traditional weaning protocols. Further studies are required to standardize the four phase approach.

AN EVALUATION OF CHILDREN'S HOSPITAL OF EASTERN ONTARIO AND THE OTTAWA HOSPITAL-GENERAL CAMPUS' GENTAMICIN DOSING PROTOCOL IN THE TREATMENT OF BACTERIAL INFECTIONS IN NEONATES

Delenam Adugu, ${ }^{1,2}$ Nisha Varughese ${ }^{1}$ Léna Lauzon, ${ }^{1}$ Régis Vaillancourt, ${ }^{1}$ Annie Pouliot, ${ }^{1}$ and Carmen $\mathrm{Ma}^{1}$ ${ }^{1}$ Children's Hospital of Eastern Ontario, Ottawa, ON, Canada, ${ }^{2}$ University of Nottingham, Nottingham, UK apouliot@cheo.on.ca

RATIONALE: Children's Hospital of Eastern Ontario (CHEO) and The Ottawa Hospital have designed a gentamicin dosing protocol to provide standardized treatment for bacterial infections in neonates. However, recent observations suggest the current protocol may lead to toxic trough concentrations (troughs $>2 \mathrm{mg} / \mathrm{L}$ ), especially in preterm and hypoxic term neonates. As a result, a review of current gentamicin dosing guidelines will be completed to identify modifications, if necessary, to current dosing guidelines. The primary goal of the amendment is to target trough gentamicin concentrations below $2 \mathrm{mg} / \mathrm{L}$. This retrospective chart review will take place exclusively at CHEO and at The Ottawa Hospital Campuses. OBJECTIVE: To evaluate and review the current gentamicin dosing protocol for toxicity by determining the incidence of inappropriately high trough gentamicin levels (troughs greater than $2 \mathrm{mg} / \mathrm{L}$ ) in neonates receiving gentamicin. Methods: This study was a retrospective chart review includes neonates admitted between June 1st 2012 and December 31st 2012 to the neonatal intensive care unit and who have been prescribed gentamicin. Neonates without pregentamicin levels were excluded from this study. Eligible neonates were divided into age categories based on gestational and postnatal age, to reflect the age categories used in the current protocol. Baseline characteristics, the time and the date of gentamicin initiation and discontinuation, dose and frequency of gentamicin administration and levels were all noted for all neonates in each category. RESULT: We identified 168 neonates who were eligible. $57(33.9 \%), 59(35.1 \%)$ and 52(31\%) came from CHEO, TOH and Civic, respectively. 37 out of 168 neonates had high trough levels (troughs $>2 \mathrm{mg} / \mathrm{L}$ ); $65 \%, 32 \%, 3 \%$ of these neonates were from $\mathrm{CHEO}, \mathrm{TOH}$ and $\mathrm{Civic}$ respectively. 34 out of the 37 neonates with toxic trough levels (>2 mg/L) were all on dosing frequency of q $18 \mathrm{hr} .4$ out the 34 on q $18 \mathrm{hr}$ should have been on q $24 \mathrm{hr}$ as per the current protocol; their gestational age $<28 \mathrm{wk}$ and PNA $<14 \mathrm{~d}$. Dosing interval of $\mathrm{q} 18 \mathrm{hr}$ was mainly used in neonates $29-36 \mathrm{wk}$ PNA $<14 \mathrm{~d}$. In general, troughs greater than $2 \mathrm{mg} / \mathrm{L}$ were mainly observed in neonates who were $<14 \mathrm{~d}$ and who fall into the following age categories: 29-30 wk, 31-32 wk, 33-36 wk, >37 wk. CONCLUSION: These results indicate that toxic gentamicin trough levels $(>2 \mathrm{mg} / \mathrm{L})$ were mainly seen in neonates who are on $\mathrm{q} 18 \mathrm{hr}$ and who fall into the gestational age $29-30 \mathrm{wk}$, with a PNA of $<14 \mathrm{~d}$. Following this, the current protocol needs modification to account for this.

\section{SMART SYRINGE PUMP MONITORING}

Carmen Ma, Régis Vaillancourt, Meechen Tchen, Mary MacNeil, Dan Collins, and Kim Greenwood Children's Hospital of Eastern Ontario, Ottawa, ON, Canada apouliot@cheo.on.ca

BACKGROUND: There is a scarcity of evidence on how organizations have used "smart pump" 
technology to inform their practices and improve infusion safety. In 2010, the Children's Hospital of Eastern Ontario (CHEO) was the first organization in North America to introduce a networked 802.11g wireless syringe pump with smart pump technology to provide immediate infusion data for analysis. Aims: This presentation will explain the interdisciplinary collaborative process that was established to track, monitor and use pump data to make improvements both in drug library settings as well as support direct practice improvements. METHODS: Every month, the pharmacist downloads the information from the wireless pumps and assesses the compliance with the drug library and potential causes leading to compliancy less than $95 \%$ are investigated. In addition, programmed infusions are assessed to see whether limits are reached and whether these overrides were legitimate. All issues are discussed with members of the interprofessional team building on processes and relationships established during the implementation phase. Regularly scheduled updates allow additions of new drugs or modifications of limits. RESULTS: From January 1st, 2011 to December 31st, 2011 a total of 57,804 infusions were run on the pump. Of these, $54,664(94.5 \%)$ infusions were run within the drug library and $51,480(89 \%)$ of the infusions had no safety events. CONCLUSION: In summary, having a rigorous monitoring system coupled with interprofessional collaboration ensures smart pumps deliver on their promise to contribute to patient safety related to infusion therapy practices.

\section{ONCE DAILY CEFTRIAXONE AND METRONI- DAZOLE FOR PERFORATED APPENDICITIS IN CHILDREN}

Theresa Ringenberg, ${ }^{3}$ Christine Robinson, ${ }^{2,3}$

Rachel Meyers, ${ }^{1,3}$ and Suzannah Kokotajlo ${ }^{2}$

${ }^{1}$ Saint Barnabas Medical Center, Livingston, NJ, USA, ${ }^{2}$ Atlantic Health System, Morristown, NJ, USA, ${ }^{3}$ Rutgers, The State University of New Jersey, Piscataway, NJ, USA tmringenberg@gmail.com

INTRODUCTION: The 2010 joint guidelines by the Surgical Infection Society and the Infectious Diseases Society of America recommendations for complicated intra-abdominal infections currently include ceftriaxone plus metronidazole as an appropriate regimen for empiric treatment in both pediatric and adult patients. The adult dosing recommendations include the option for once daily metronidazole 1.5 grams, however, the recommended pediatric dosage for metronidazole is 30 to 40 $\mathrm{mg} / \mathrm{kg} /$ day divided every 8 hours, likely due to minimal evidence supporting once daily use in children. Once daily ceftriaxone and metronidazole has been shown in a study with a small number of pediatric patients to be equally efficacious and more cost effective in preventing infections compared to another recommended empiric regimen of ampicillin, clindamycin and gentamicin (each medication is given 3 to 4 times daily). The purpose of this study is to identify a large cohort to support the safe and effective use of once daily metronidazole and ceftriaxone for perforated appendicitis in pediatric patients. METHODS: A retrospective chart review was conducted to identify pediatric patients with perforated appendicitis between January 1, 2007 to October 1, 2012 who received once daily ceftriaxone and metronidazole. Children 18 years of age and younger with evidence of perforated appendicitis (as documented on CT or visualization) receiving once daily ceftriaxone $50 \mathrm{mg} /$ $\mathrm{kg}$ and metronidazole $30 \mathrm{mg} / \mathrm{kg}$ for minimum of four days were included. Outcomes evaluated included length of stay, length of intravenous antibiotic therapy, maximum temperature for each of the five days of antibiotics, abscess rate in the two months following surgery, medication charges and adverse effects. RESULTS: Ninety eight patients were included and received an average of 5.7 days of once daily ceftriaxone and metronidazole. Post-operative abscesses developed in $10 \%$ of patients at an average of 9.8 days after the appendectomy. Of the patients who developed an abscess, the average duration of antibiotic therapy prior to developing the abscess was 6 days (range 4 to 10) and $30 \%$ of them were previously discharged on antibiotics. Defervescence occurred by post-operative day 2 on average. Ceftriaxone and metronidazole therapy was well tolerated, with no evidence of renal or hepatic dysfunction with either therapy. CONCLUSION: Once daily ceftriaxone and metronidazole appears to be a safe and effective option for pediatric patients with perforated appendicitis who undergo an appendectomy following initial diagnosis. Due to limitations of the study, a large scale prospective trial with a comparator group is necessary to confirm the results of this study.

\section{COMPARISON OF THREE BODY SIZE DESCRIP- TORS IN CRITICALLY ILL OBESE CHILDREN AND ADOLESCENTS: IMPLICATIONS FOR MEDICATION DOSING}

Emma L. Ross, Jennifer M. Jorgensen, Peter E. Dewitt, Carol Okada, and Pamela D. Reiter

Children's Hospital Colorado, Aurora, Colorado, USA emma.ross@childrenscolorado.org

BACKGROUND: Most clinicians agree that ideal or lean body weight should be used to calculate maintenance doses of medications in obese children. Calculation of these body size descriptors, however, can be difficult. The objective of this study was to describe the relationship between three methods of weight determination in obese children and discuss feasibility of bedside use for routine care. METHODS: This was an organizational research risk and quality improvement study evaluating patients admitted to a 26 bed pediatric intensive care unit (PICU) within a tertiary care Children's Hospital. 
Patients were eligible for inclusion if they were admitted to the PICU during a 13-month period (July 2010- July 2011) and were older than 2 years. Age, weight, height, and gender were extracted from the electronic medical record. Patients identified as obese ( $\geq 95$ th percentile body mass index (BMI) for age) and severely obese ( $\geq 99$ th percentile BMI for age) were included in the weight determination portion of this study. Lean body mass (LBM) estimation, using previously validated complex equations derived by the Peters and Foster Methods, was compared to ideal body weight (IBW) estimates using the simple 50th percentile BMI method. A descriptive analysis was performed on each variable. Mean \pm standard deviations (and range) are presented. To assess the absolute differences between the values generated by the three methods, intraclass correlation (ICC) and Bland-Altman plots were performed. RESULTS: A total of 1,369 patients met initial study inclusion criteria and 178 patients met criteria for the weight determination portion (age $=9.28 \pm 5$ years (2-20); actual weight $55.5 \mathrm{~kg} \pm 33.9 \mathrm{~kg}(11-141) ; 46 \%$ female). Sixty patients were defined as severely obese, 118 were defined as obese. The ICC between the three methods was 0.968 (95\% CI: 0.959, 0.975). The Peters method tended to estimate higher weights than the Foster or BMI method. Bland-Altman plots illustrated good agreement between methods in children $<50 \mathrm{~kg}$. However, method agreement began to diverge above $50 \mathrm{~kg}$ and was influenced by gender. CONCLUSION: The three methods used to determine IBW / LBM in critically ill obese children older than 2 years of age demonstrated strong correlation and acceptable agreement. Systematic biases were identified in children $>50 \mathrm{~kg}$ where variance was higher. This suggests that these methods are not interchangeable at the highest end of the weight range. The BMI method tended to estimate lower weights in children $>50 \mathrm{~kg}$ and is therefore the safest body size descriptor for medication dosing in high risk patients. Since superiority of one method was not demonstrated, ease of clinical use becomes the driving factor for daily application. Of the three methods, the BMI Method is the most feasible, allowing for seamless integration into the daily workflow of the clinical pharmacist.

EVALUATION OF ESSENTIAL FATTY ACID DEFICIENCY IN PEDIATRIC PATIENTS RECEIVING INTERMITTENT FAT EMULSION DURING PRODUCT SHORTAGES

Chasity Shelton and Catherine Crill

The University of Tennessee Health Science Center, Memphis, TN, USA, Le Bonheur Children's Hospital, Memphis, TN,USA cshelton@uthsc.edu

INTRODUCTION/OBJECTIVE: There have been periodic shortages of intravenous fat emulsion (IVFE) since 2009. Since our institution does not repackage
IVFE, we have attempted to address the IVFE product shortages by giving IVFE less frequently based on patient weight $(<1 \mathrm{~kg}$ : daily; $\geq 1 \mathrm{~kg}$ and $<3.5 \mathrm{~kg}$ : 5 days/week; $\geq 3.5 \mathrm{~kg}$ and $<5 \mathrm{~kg}$ : 3 days/week; $\geq 5$ kg: 2 days/week; age $\geq 1$ year: once weekly). During intermittent IVFE dosing, patients were monitored for signs of essential fatty acid deficiency (EFAD) and some patients had essential fatty acid assessment. The purpose of this study was to assess the incidence of essential fatty acid abnormalities in pediatric patients receiving intermittent IVFE dosing. METHODS/DESIGN: Subjects were identified through screening a generated report from the electronic medical record of all patients with a comprehensive fatty acid panel from November 2009 through February 2013. Patients were included if they were receiving parenteral nutrition $(\mathrm{PN})$ with intermittent IVFE dosing or no IVFE at the time of the fatty acid assessment. An unpaired t-test and descriptive statistics were used for data analysis. A p-value of $<0.05$ was considered significant. RESULTS: Data are reported as mean \pm SD; range. Fifteen patients were included in the analysis (age 3.5 \pm 5.7 years; 19 days-17.8 years; weight $11.4 \pm 14.3 ; 2.2-60 \mathrm{~kg}$; percent of enteral calories $19 \pm 19 ; 0-51)$. Five patients had results consistent with EFAD (weekly IVFE dose $1.8 \pm 1.5 ; 0-3.6 \mathrm{~g} / \mathrm{kg}$; percent of goal calories from IVFE $3 \pm 3 ; 0-6)$. Days per week of IVFE administration were $0(n=1), 1(n=3)$, and $3(n=1)$. In the 10 patients with normal fatty acid profiles (weekly IVFE dose $5 \pm 3$; 0-9.1 $\mathrm{g} / \mathrm{kg}$; percent of goal calories from IVFE $7 \pm 4 ; 0-13)$, days per week of IVFE administration were $0(n=1), 2$ $(n=2), 3(n=6)$, and $5(n=1)$. The calculated p-value between groups for the weekly IVFE dose was $<0.05$. One patient was not receiving IVFE in both groups (normal and abnormal fatty acid profile) prior to essential fatty acid assessment. Both patients were receiving $50 \%$ of goal calories from enteral nutrition. The patient with an abnormal fatty acid profile is a home PN patient with a reported history of protracted emesis for 2 weeks at home prior to assessment in the hospital. CONCLUSIONS: In this study, patients receiving an average daily IVFE dose of $0.7 \mathrm{~g} / \mathrm{kg}$ were able to maintain essential fatty acid status, whereas those receiving an average daily dose below $0.3 \mathrm{~g} / \mathrm{kg}$ resulted in deficiency. Our findings are consistent with published literature that suggests a minimum of $0.5 \mathrm{~g} / \mathrm{kg} /$ day IVFE is required to prevent EFAD. Intermittent dosing could be considered an option for clinicians during IVFE shortages. Periodic assessment of essential fatty acid status is recommended in all patients receiving intermittent IVFE or daily doses $\leq 0.5 \mathrm{~g} / \mathrm{kg} /$ day.

\section{SYSTEMIC EFFECTS OF OPHTHALMIC CYCLO- PENTOLATE IN A MODEL OF RETINOPATHY OF PREMATURITY}

Nicole Shumiloff, ${ }^{1,2}$ Suraporn Matragoon, ${ }^{1}$

StephanieGoei, ${ }^{2}$ Kalen Manasco, ${ }^{1,2}$ and Azza El-Remessy ${ }^{1}$ 
${ }^{1}$ University of Georgia College of Pharmacy, Augusta, Georgia, USA, ${ }^{2}$ Georgia Regents Medical Center, Augusta, Georgia, USA nshumiloff@gru.edu

INTRODUCTION: Cyclopentolate is standardly used in ophthalmologic examinations of neonates to facilitate screening for retinopathy of prematurity (ROP). Although these examinations are considered benign, reports of systemic effects in adults and children throughout the past several decades have raised serious concerns. One of the greatest impacts seen in the neonatal population from systemic adverse effects of ophthalmic cyclopentolate instillation is post-administration feeding intolerance. The goal of this project was to evaluate systemic concentrations of cyclopentolate after ophthalmic administration, and to examine and establish the correlation between systemic concentrations and effects on feeding in a neonatal rodent model. METHODS: Neonatal mice were randomized into two groups to simulate a neonatal model for ophthalmic medication administration. Group A received tetracaine $0.5 \%$, cyclopentolate $0.5 \%$, and phenylephrine $2.5 \%$ ophthalmologic solutions in accordance with the protocol used for ROP examinations at the Children's Hospital of Georgia, while group B received the same protocol with placebo (saline) in place of the ophthalmic cyclopentolate. Serum samples to measure systemic concentrations of cyclopentolate post-ophthalmic administration were assessed at four time points $-0.5,1,24$ hours, and 7 days. Baseline and daily weights were also obtained to evaluate impact on feeding intolerance and weight gain. The primary outcome measure of this study was the progression of weight gain after administration of ophthalmic cyclopentolate or placebo. Correlations between systemic concentrations of cyclopentolate and weight gain compared to placebo were also conducted. RESULTS: Analysis of serum levels demonstrated absorption of cyclopentolate into systemic circulation after ophthalmic administration, with significant cyclopentolate levels detected at 30 minutes (116 $\mathrm{ng} / \mathrm{mL}), 1$ hour (60 ng/mL), and 24 hours (6.2 ng/ $\mathrm{mL}$ ) after instillation. There were also differences in weight gain observed on the days following drop administration between groups A and B, with group B (placebo) having significantly more weight gain at days 3 and 7 compared to baseline $(p=0.2)$. CONCLUSIONS: Ophthalmic cyclopentolate administration produces significant and detectable concentrations in serum samples, indicating rapid systemic absorption. Mice receiving cyclopentolate were found to have decreased weight gain compared to mice receiving placebo, with the greatest differences seen on days 3 and 7 post-administration, suggesting a correlation with systemic cyclopentolate levels and feeding intolerance. Impact on poor weight gain in the human population may be significantly greater as patients are examined for retinopathy of prematurity on a weekly basis. Pre-clinical results provide rationale for further studies in patients.

\section{PHARMACOECONOMIC IMPACT OF SURFAC- TANT SELECTION ON MORBIDITY IN REINTU- BATED INFANTS}

Phillip Simmons,${ }^{1}$ Fernando Moya, ${ }^{2}$ Sunil Sinha, ${ }^{3}$ and Russell Clayton ${ }^{1}$

${ }^{1}$ Discovery Laboratories, Inc., Warrington, PA, USA, ${ }^{2}$ Coastal Carolina Neonatology, Wilmington, NC, USA, ${ }^{3}$ James Cook University Hospital, Middlesbrough, UK psimmons@discoverylabs.com

INTRODUCTION: A recent post hoc analysis of data from two surfactant clinical trials comparing surfactant therapies demonstrated that up to $47 \%$ of infants extubated after surfactant replacement therapy required reintubation, and infants requiring reintubation were at significantly higher risk for major morbidities. A difference in reintubation rates between surfactant treatment groups was also observed and was used to develop a pharmacoeconomic model. OBJECTIVE: To estimate the in-hospital cost impact of major morbidities in reintubated neonates. Methodology: The rate of morbidities and reintubation were determined from 1546 preterm infants enrolled in two clinical trials comparing lucinactant with surfactants beractant or poractant alfa. Inpatient hospital costs for treating neonates with the primary diagnoses of BPD, air leak, sepsis, NEC, IVH, and a composite of all diagnoses combined were obtained from an established healthcare cost database. The average cost per infant was estimated by multiplying the reintubation rate, the incidence of each morbidity, and the cost for treatment of each morbidity. Costs were compared between surfactant therapies on per-infant costs, standardized to 100 infants. RESULTS: The rate of reintubation in the lucinactant group was $8 \%-15 \%$ lower than the rate of reintubation in the beractant or poractant alfa groups. Based on these relative differences in reintubation rates, the resultant average projected cost difference associated with reintubation, standardized to 100 infants, ranged from $\$ 42,726$ to $\$ 392,371$, with a composite of $\$ 157,894$. CONCLUSION: The occurrence of major morbidities associated with reintubation in infants treated with surfactant replacement therapy has a potentially profound impact on healthcare resource utilization in the neonatal population.

\section{DEVELOPMENT AND IMPLEMENTATION OF A NURSE DRIVEN ELECTROLYTE REPLACEMENT PROTOCOL IN A PEDIATRIC CRITICAL CARE UNIT}

Julie Sinclair-Pingel, Michelle Terrell, Fred Hargrove, and Alison Grisso

Monroe Carell Jr. Children's Hospital at Vanderbilt, Nashville, TN, USA julie.sinclair@vanderbilt.edu 
Electrolyte replacement is a standard of care in Pediatric Critical Care Units. The diuretic therapy required by this patient population often leads to low electrolyte levels requiring repletion. Factors such as current electrolyte level, dose and type of diuretic, type of IV access, concentration of the replacement electrolyte and renal function must be considered when replacing electrolytes. Although computerized prescriber order entry (CPOE) and decision support for electrolytes had been in place at our institution for several years, electrolyte replacement was nonstandardized and time consuming. Prior to implementation, electrolyte replacement was often initiated by the bedside nurse who notified the prescriber of a low electrolyte level. The prescriber would assess the patient, determine the need for replacement therapy and enter electrolyte doses and labs at their discretion. Large amounts of time were spent managing electrolyte imbalances with a process that was highly variable. Our goal was to empower the bedside nurse, save prescriber time and improve patient safety by standardizing doses and subsequent labs. The most commonly used electrolytes; potassium chloride, calcium gluconate, calcium chloride and magnesium sulfate were included in the protocol. A CPOE ordering page was created for each electrolyte in the protocol. Each electrolyte page provided information on which patients could be included in the protocol and excluded patients with poor renal function. The order pages were designed with several features which included the most recent trend in electrolyte values, standardized doses of each electrolyte and standardized future lab orders. Because the appropriate dose may be dependent on the electrolyte value, only the dose appropriate for the current electrolyte value could be ordered. Repeat labs were also automatically ordered based on the pre-replacement electrolyte level and the replacement dose given. Prior to implementation of the new CPOE ordering pages, nursing education on the new process was provided. With the new process, each patient is assessed daily to determine if they meet criteria and would benefit from the protocol. The prescriber then enters an order for the "Pediatric Electrolyte Replacement Protocol." The prescriber selects which electrolytes may be replaced in this initial ordering. The order stays active for only 24 hours to force daily reevaluation of each patient. If a patient has an active electrolyte replacement protocol order, the bedside nurse may enter the CPOE system to request replacement electrolytes in preset, standardized doses based on current lab values. The prescriber does not have to be contacted to request doses. The protocol has been well received by prescribers, bedside nurses and pharmacists. It has allowed for safe electrolyte replacement and appropriate monitoring without delays in care.
ANALYSIS OF CURRENT PROTOCOL FOR THE USE OF METHADONE IN TREATING NEONATAL ABSTINENCE SYNDROME

Judith Lorenzo, ${ }^{2}$ Anita Siu, ${ }^{1,2}$ Yen-Hong Kuo, ${ }^{3}$ and Elizabeth Assing ${ }^{2}$

${ }^{1}$ Rutgers University, Piscataway, NJ, USA,${ }^{2} \mathrm{~K}$. Hovnanian Children's Hospital at Jersey Shore University Medical Center, Neptune, NJ, USA, ${ }^{3}$ Jersey Shore University Medical Center, Neptune, NJ, USA anitasiu@pharmacy.rutgers.edu

PURPOSE: The purpose of this study is to evaluate our Neonatal Intensive Care Unit's current protocol for the usage and dosing of methadone in the management of neonatal abstinence syndrome (NAS). METHOD: This study is a retrospective and prospective chart review in neonates diagnosed with NAS. Primary outcome measurement includes the number of methdone doses needed during the first 24 hours to achieve a Finnegan score of $\leq 8$. The secondary outcome measurement is the compliance of methadone protocol. Inclusion criterias were gestational age 34-40 weeks, mothers using methadone or any other opiod during pregnancy, and neonates treated solely with methadone. Exculsion criteria included neonates who had received phenobarbital or paregoric. Data collection included age, sex, mode of delivery, weight, race, APGARS, maternal methadone dose and use of other substances, neonate's age at the time of methadone initiation, Finnegan scores, mode of feeding, deviation of methadone protocol, and duration of treatment. RESULTS: From November 2010 to December 2011, Twenty patients were treated and included for this study. Seventeen out of the $(85 \%, 95 \%$ confidence interval $[62 \%, 97 \%]$ were treated according to the protocol. Results show that 3 of the patients deviated from the protocol at various points in their treatment course. Some of the data collection included age, sex, mode of delivery, weight, race, APGARS, maternal methadone dose and use of other substances, neonate's age at the time of methadone initiation, Finnegan scores, mode of feeding, deviation of methadone protocol, and duration of treatment did not seem to affect doses needed. None of the subjects required more than 6 doses within the first 24 hours to achieve a Finnegan score of $\leq 8$. There were no adverse events due to methadone treatment CONCLUSION: The use of our current protocol demonstrates the efficacy and safety from this studied patient population.

\section{SIMULTANEOUS IMPLEMENTATION OF AN INTEGRATED ELECTRONIC MEDICAL RECORD (EPIC) AND BARCODE MEDICATION ADMINIS- TRATION (BCMA) PROCESS IN A CHILDREN'S HOSPITAL}

Julie Slomkowski, Jessica Carona, Sarah Kelly, and Marjorie Lazarre

Yale-New Haven Hospital, New Haven, CT, USA julie. slomkowski@ynhh.org 
BACKGROUND: With recent advances in healthcare technology, the expectation to integrate the inpatient and outpatient medical record has become more prominent. At Yale-New Haven Children's Hospital $(\mathrm{YNHCH})$, the implementation of Epic bridged this gap between patient care settings with the use of a single system. Additionally, the need to maximize patient safety has led to the implementation of bedside barcode medication administration (BCMA). Although several institutions have undergone this process, little to no published literature exists regarding successful conversion in a children's hospital. Due to the unique intricacies of pediatric and neonatal medication therapies, it is necessary to tailor the conversion process to suit the quality and safety expectations of each institution. This project has outlined the preparation, build, validation, challenges encountered, and resolutions accepted throughout the process of implementing Epic and BCMA at $\mathrm{YNHCH}$. METHODS: A multi-disciplinary group collaborated to standardize the medication formulary across the health system and to assign barcodes to every medication formulation. The medication database was imported into Epic for review by pediatric pharmacists to verify, optimize, and validate: dose, frequency, dose calculation and rounding, route, formulation, preparation, and dispense logic. After all medication records had been validated, a gap analysis of order set nomenclature and appropriateness was performed. Education and training with regards to bedside barcoding and system use was given to all health care providers prior to system implementation. Following system go-live, review and optimization of Epic medication records occurred, with suggested resolutions sent to system representatives. RESULTS: Data collected showed that on day one of system implementation, utilization of beside barcode scanning was estimated to be $83 \%$, far exceeding the set target of $60 \%$. In the 30 days following system go-live, it was estimated that the patient was scanned before medication administration $86.4 \%$ of the time, while the medication was scanned before administration $80.9 \%$ of the time. The number of medication-related events which could potentially have been prevented by bedside barcoding was also analyzed. Prior to Epic implementation, an average of 3.6 events per month occurred, while in the month after go-live, one event occurred. In addition, reported medication delays were also decreased. Fewer errors in discharge instructions due to the more comprehensive medication reconciliation aspect of the system are expected, but yet to be quantified. CONCLUSIONS: Simultaneous implementation of an integrated electronic medical record and BCMA in a children's hospital to advance patient safety and quality care requires a multi-disciplinary approach. Significant involvement from pharmacy staff in verification and validation of medication records is necessary.
EVALUATING TREATMENT OPTIONS FOR NAP1 VERSUS NON-NAP1 STRAINS OF CLOSTRIDIUM DIFFICILE INFECTION AMONG PEDIATRIC PATIENTS AT AN ACADEMIC HOSPITAL

Amelia Smith, Kathyrn Matthias, and Hanna Phan University of Arizona, Tucson, AZ, USA, University of Arizona Medical Center, Tucson, AZ, USA asmith@pharmacy.arizona.edu

INTRODUCTION/OBJECTIVE: The incidence of Clostridium difficile infections (CDI) in pediatric patients has increased over the years. Increased prevalence could be associated with the emergence of a hyper virulent strain, specifically ribotype 027 (B1/ NAP1/027). Because of this emerging strain, a broader spectrum of patient populations are considered to be at higher risk for infection. The objectives of this study were to: 1) evaluate the incidence of B1/NAP1/027 versus non-B1/NAP1/027 strains of CDI among pediatric patients, 2) compare treatment(s) prescribed, treatment duration, rate of infection recurrence based on strain type and severity scoring, rates of re-infection or recurrence, and treatment failures among pediatric patients, 3) evaluate incidence of CDI and treatment practices in children greater than 6 months old to 18 years of age, and 4) describe incidence of testing for Clostridium difficile toxin and evaluate therapy if used among patients less than 6 months of age. METHODS/DESIGN: This was a retrospective study of pediatric patients ( $0-18$ years old) admitted to an academic medical center who had detection of Clostridium difficile toxin reported between April 1, 2009 and July 1,2011. Patients were excluded if they were inpatient at the academic medical center for less than 48 hours or if they did not receive metronidazole or oral vancomycin as part of CDI treatment. Data collected included demographics, Clostridium difficile toxin assay results and other laboratory data, CDI therapy and other medications prescribed, interventions, CDI outcomes, and complications associated with CDI or therapy. Data analyses included descriptive and inferential statistics to examine data including demographics, strain type and severity of infections, and treatment failures among patients. General linear model analyses were performed to determine risk factors for incidence of CDI, such as prior medication therapy, nutrition, co-morbid conditions, prior surgery, prior hospital admission, and immunocompromised status. RESULTS: A total of 45 pediatric patients with Clostridium difficile toxin detection were included in study analyses. The median age was 6.2 years with a range of 0.31 to 17.9 years. Oral or intravenous metronidazole was prescribed as initial therapy in $89 \%$ of the patients. Strain type was available in $77 \%$ of patients, with B1/NAP1/027 detected in $31 \%$ of stool samples tested. Within the first 21 days after initial toxin detection, there was a $13 \%$ rate of clinical failure or death, although none directly associated 
with CDI. Within days 22 through 65 after initial toxin detection, there was a $16 \%$ rate of recurrence or reinfection of Clostridium difficile associateddiarrhea. Initial therapy selection, therapy duration, and rate of recurrence or reinfection were not significantly associated with B1/NAP1/027 strain type. CONCLUSION: Despite variability in severity of infection, the majority of pediatric patients with CDI were treated with metronidazole and were infected with a non-B1/NAP1/027 strain type.

\section{THE ROLE OF THE CLINICAL PHARMACIST IN THE EMERGENCY DEPARTMENT OF A PEDIAT- RIC ACADEMIC HEALTH SCIENCE CENTER: A PILOT PROJECT}

Sandra Bjelajac-Mejia, ${ }^{1,2}$ Lori Chen, ${ }^{1,2}$ Sabrina Boodhan,,$^{1,2}$ Michael Pe,,$^{1,2}$ Jennifer Poh, ${ }^{1,2}$ Marina Strzelecki, ${ }^{1,2}$ and James Tjon ${ }^{1}$

${ }^{1}$ Hospital for Sick Children, Toronto, Canada, ${ }^{2}$ University of Toronto, Toronto, Canada marina.strzelecki@sickkids.ca

The emergency department (ED) at the Hospital for Sick Children (Toronto, Canada) treats approximately 50,000 children per year. Pharmacy services in the ED are currently limited to provision of ward stock medications and auditing selected medication orders that require preparation and dispensing from the inpatient pharmacy. Since the majority of medications are ward stock in the ED, prescribing, dispensing and administration of medications at the point of care frequently occurs without prior review by a pharmacist. This creates a situation whereby preventable medication errors may occur. Clinical pharmacy services at the Hospital for Sick Children have been established in various inpatient and outpatient areas for over 20 years. Due to the recognized value of a clinical pharmacist in existing inpatient areas, a collaborative effort between the ED and the Pharmacy Department to pilot a clinical pharmacist in the ED was proposed. The objectives of the pilot project were to: 1) identify and characterize the medication-related needs of patients and ED staff; 2) provide support for the medication reconciliation process; 3 ) assess the impact of the clinical pharmacist on patient care; and 4) define the role of the clinical pharmacist and suggest an implementation plan. The pilot project was implemented over four weeks between July and November of 2012. Three clinical pharmacists currently working on Paediatric and Respiratory Medicine teams participated in the pilot project and were involved in the care of 158 patients throughout the 4 -week period. During the pilot, $60 \%$ of the time was spent on direct patient care activities. The majority of this time (94\%) involved patient assessments, answering drug information questions, medication reconciliation, and identification and resolution of drug therapy problems (DTPs). Other activities included: medication counseling, therapeutic drug monitoring, and providing seamless care on transfer to an inpatient ward. A total of 40 DTPs were identified during the pilot whereby $27(67 \%)$ of those were actual DTPs and 13 (33\%) were potential DTPs. The most commonly identified DTP was the need for additional drug therapy. Forty percent of the time was devoted to non-direct patient care activities such as staff education and quality improvement projects including: re-organization of resuscitation carts, providing feedback on development of appendicitis order sets, and delivering an in-service on recently updated febrile neutropenia guidelines. Based on the pilot project, there appears to be a role for a clinical pharmacist in both direct and non-direct patient care activities. Pharmacists can play a valuable role in patient and medication safety by serving as a resource to both patients and health care staff in the fast-paced environment of a pediatric emergency department.

\section{SENSITIVITY AND SPECIFICITY OF DOSING ALERTS FOR DOSING ERRORS AMONG PEDI- ATRIC PATIENTS}

Jeremy Stultz, ${ }^{1,2}$ Kyle Porter, ${ }^{3}$ and Milap Nahata ${ }^{1,2}$ ${ }^{1}$ The Ohio State University College of Pharmacy, Columbus, $\mathrm{OH}, \mathrm{USA},{ }^{2}$ Nationwide Children's Hospital, Columbus, OH, USA, The Ohio State University Center for Biostatistics, Columbus, OH, USA stultz.31@osu.edu

INTRODUCTION: Dosing alerts, based on information technology database dose ranges, are being used in pediatric institutions to prevent medication dosing errors and adverse events. However, sensitivity and specificity of dosing alerts for dosing errors have not been analyzed. OBJECTIVES: The main objectives of this study were to determine the sensitivity and specificity of a dosing alert system for dosing errors and to compare the sensitivity of a proprietary system (First Databank) with and without institution specific customization. Secondary objectives were to determine the severity of dosing errors and to identify areas for dosing alert improvement. METHODS: A retrospective analysis of medication orders causing dosing alerts, reported adverse drug events, and dosing errors was conducted during July, 2011 at a tertiary pediatric hospital. Patient medical records were reviewed, associated orders were compared with Lexi-Comp and institutional dose ranges, and dosing errors were classified by severity. Dosing alert sensitivity with customized and non-customized ranges was compared using McNemar's test. RESULTS: There were 47,181 inpatient orders during the studied period; 257 dosing errors were identified $(0.54 \%)$. The sensitivity of alerts for dosing errors was $54.1 \%$ (CI: 47.8-60.3) if customization had not occurred and increased to $60.3 \%$ (CI: 54.0-66.3) with customization $(\mathrm{p}=0.02)$. The sensitivity of dosing alerts for underdoses was $49.6 \%$ if customization had not occurred and $60.3 \%$ with customization $(p=0.01)$. Specificity of the customized alerts for dos- 
ing errors was 96.2\% (CI: 96.3-96.6) with a positive predictive value of $8 \%$ (CI: 7.0-9.6). All orders with alerts were overridden by $\geq 1$ practitioner and $41 \%$ of dosing errors reached the patient. Six dosing errors caused a laboratory monitoring intervention or an adverse event, with 2 having alerts. Lack of indication specific dosing ranges was the most common reason why an alert did not occur for a dosing error. CONCLUSIONS: Dosing alerts had a low sensitivity and positive predictive value for dosing errors, but may have prevented dosing errors from reaching patients. Customization increased the sensitivity of alerts for dosing errors. Changes in dosing ranges should be implemented to improve the sensitivity and positive predictive value of dosing alerts to decrease alert fatigue.

\section{PEDIATRIC VANCOMYCIN DOSING AND MONI- TORING: DO PROTOCOLS IMPROVE COMPLI- ANCE WITH CURRENT RECOMMENDATIONS?}

Lindsay Svehla, Missy Ochalla, and

Malliswari Challapalli

Ronald McDonald Children's Hospital Loyola University Medical Center, Maywood, IL, USA lindsay.svehla@gmail. com

OBJECTIVES: The purpose of this project was to achieve goal therapeutic vancomycin levels as recommended by the IDSA using their dosing guidelines and to decrease the number of dose adjustments needed to achieve goal therapeutic levels. BACKGROUND: Infectious Diseases Society of America (IDSA) published clinical practice guidelines in 2011 for the treatment of methicillin-resistant Staphylococcus aureus (MRSA) infections. These guidelines provide recommendations regarding dosing and therapeutic monitoring of vancomycin for the treatment of MRSA infections in pediatric patients. METHODS: Baseline data was collected retrospectively by reviewing patient charts. From this data it was determined that goal vancomycin troughs were not consistently being achieved with our current regimens and vancomycin troughs were not frequently being obtained at steady state. The new IDSA dosing guidelines for vancomycin was then implemented by adding new order help text and dosing buttons into our physician order entry screens and by performing education to the physicians. After implementation, prospective data was collected and analyzed to evaluate the new dosing regimen and appropriateness of obtaining vancomycin trough levels. RESULTS: Twenty-five patients received vancomycin during the prospective study period. Eighty-eight percent received the new recommended dosing regimen initially. Vancomycin trough levels were measured in 13 patients. Of these patients, $100 \%$ had trough levels drawn prior to the 4 th dose or later. Overall, $69 \%$ of patients had serum trough levels $>10 \mathrm{mg} /$ Lat steady state.
CONCLUSION: Development of the new pediatric vancomycin physician order entry screen improved physician compliance with the IDSA recommendations for pediatric patients. Increasing physician compliance with the new recommended dosing regimen lead to the following: therapeutic goal trough levels sooner with less dose adjustments required; decreased number of trough levels drawn per patient; and less patient blood draws.

\section{PEDIATRIC CURRICULUMS OF COLLEGES OF PHARMACY IN THE UNITED STATES AND CANADA \\ Timothy Todd ${ }^{1}$ and Manali Shah ${ }^{2}$ \\ ${ }^{1}$ Midwestern University Chicago College of Pharmacy, Downers Grove, IL, USA, ${ }^{2}$ Edward Hines, Jr. VA Hospital, Hines, IL, USA ttoddx@midwestern.edu}

INTRODUCTION/OBJECTIVE: In 2005 the Pediatric Practice and Research Network of the American College of Clinical Pharmacy (ACCP Peds PRN) published an opinion paper reviewing the status of and proposing standards for pediatric pharmacy education. No published investigations have evaluated compliance with the proposed standards at this time. The objective of this project was to evaluate the current status of didactic and experiential pediatric curriculums for Accreditation Council for Pharmacy Education (ACPE)-accredited entry-level pharmacy programs in the United States and Canada. METHODS: Key pediatric faculty or administrators were identified for each accredited college of pharmacy. A seventeen question electronic survey was administered to document the extent of required and elective pediatric content within their didactic curriculums. A separate fourteen question electronic survey gathered information from experiential education departments to determine advanced pharmacy practice experiences (APPE) allocated to pediatrics. RESULTS: The response rate for the didactic-focused survey was $33.6 \%$. The average required curriculum contains 31.4 hours of pediatric-focused topics. The topics most commonly covered in the required didactic curriculum are immunizations, pediatric infectious diseases, pediatric over-the-counter products, psychiatric disorders of childhood, and drugs in pregnancy / lactation. Of the responding schools, $58.8 \%$ offered a pediatric elective course to their students. A majority of the electives $(73.7 \%)$ were 2 credit hours with an average enrollment of 26.8 students. The topics most commonly covered in pediatric elective courses are pediatric infectious diseases, pediatric neurology, neonatal infectious diseases, fluids/electrolytes/nutrition, and neonatology. The experiential education survey yielded a response rate of $42.9 \%$. All responding schools offered a pediatric APPE opportunity, however $52 \%$ had less than twenty rotation allocations available to students per year. No responding 
college of pharmacy required a pediatric APPE. CONCLUSIONS: Colleges of pharmacy have typically met the ACCP Peds PRN proposed requirement of 25-30 hours of pediatrics within the required didactic curriculum. A lower compliance rate is seen with the recommendations regarding students being offered pediatric electives and pediatric-focused APPEs.. Although strides have been made in filling the gaps of pediatric pharmacotherapeutic education, a need for significant expansion exists with pediatric electives and experiential education.

\section{EFFECT OF HIGHER DOSE ENOXAPARIN DOS- ING GUIDELINE ON NUMBER OF ANTI-Xa LEV- ELS DRAWN IN CHILDREN'S HOSPITAL}

Terra Varner, ${ }^{1}$ Jennifer Bair, ${ }^{1}$ Shannon DeRienzo, ${ }^{1}$

McKaren Lewis, ${ }^{1}$ and Christina Piro ${ }^{1,2}$

${ }^{1}$ Palmetto Health Children's Hospital, Columbia, SC 29203,

USA, ${ }^{2}$ South Carolina College of Pharmacy, Columbia, SC 29203, USA terra.varner@palmettohealth.org

INTRODUCTION/OBJECTIVE: Pediatric literature suggests that for treatment of thromboembolic events, an initial higher dose of enoxaparin than recommended by the 2008 CHEST guidelines from the 8 th Edition of the American College of Chest Physicians Evidence-Based Clinical Practice Guidelines may be appropriate to achieve therapeutic anti-Xa levels in a more timely manner. The primary objective of this single center, unblinded prospective cohort study is to evaluate the efficacy of a higher weight and agebased dosing guideline at Palmetto Health Children's Hospital, as defined by the number of anti-Xa levels drawn until the patient is therapeutic. Secondary objectives are to evaluate the safety and efficacy of the dosing guideline by recording any adverse or thrombotic events believed to be secondary to therapy. METHODS/DESIGN: All patients being treated at Palmetto Children's Hospital who receive at least one treatment dose of enoxaparin as an inpatient and have an anti-Xa level drawn to assess therapeutic efficacy will be included for evaluation. Patients receiving enoxaparin for purposes of prophylaxis will be excluded. Each treatment dose and subsequent manner in which the dose is adjusted will be evaluated for adherence to the institutional guideline. Unexpected adverse events will be detected by monitoring vital signs, laboratory tests, and physical exams. Based on institutional retrospective data, it is hypothesized that adherence to the institutional dosing guideline will result in $70 \%$ of patients $<2$ months and $\geq 2$ months of age requiring no more than 3 and 2 anti-Xa levels to achieve a therapeutic anti-Xa level, with 40 patients needed for each age group to achieve $80 \%$ power with a significance level of 0.05 . RESULTS: To date, six patients $<2$ months of age and eighteen patients $\geq 2$ months of age have received treatment dose enoxaparin. Sixty-six percent of patients $<2$ months of age and $83 \%$ of patients $\geq 2$ months of age received enoxaparin dosing in compliance with the Palmetto Health Children's Hospital Enoxaparin Treatment Dosing Guideline. As compared to retrospective patients who received treatment dosing according to the CHEST 2008 guidelines and required an average of $3.4(2-6)$ and 3.1 (1-14) levels in patients $<2$ months and $\geq 2$ months of age, patients receiving treatment dosing according to our institutional guideline required an average of 2.3 (1-3) and 1.7 (1-4) levels in patients $<2$ months and $\geq 2$ months of age. Therapy among all patients appeared to be well-tolerated. CONCLUSION: Given results to date, dosing according to the Palmetto Health Children's Hospital Enoxaparin Treatment Dosing Guideline results in fewer anti-Xa levels needing to be drawn in order to ACHIEVE A THERAPEUTIC ANTI-XA. DATA WILL CONTINUE TO BE COLLECTED AND EVALUATED.

\section{PSEUDOMONAS AERUGINOSA AND STREPTO- COCCUS PNEUMONIAE MINIMUM INHIBITORY CONCENTRATION (MIC) DISTRIBUTION AT TWO INDIANAPOLIS HOSPITALS}

Colleen Linsenmayer, ${ }^{1}$ Kelsey Lyon, ${ }^{1}$ Stacy Smoler, Megan Veverka, ${ }^{1}$ Kristen Nichols, ${ }^{1,3}$ and

Kendra Atkinson ${ }^{1,2}$

${ }^{1}$ Butler University, Indianapolis, IN, USA, ${ }^{2}$ Wishard Health Services, Indianapolis, IN, USA, ${ }^{3}$ Riley Hospital for Children, Indianapolis, IN, USA mveverka@butler.edu

BACKGROUND: Bacterial minimum inhibitory concentrations (MICs) correlate with clinical outcomes, making it important to be aware of local microorganism susceptibilities. While the categorization of an organism as susceptible or resistant may aid the prescriber in selecting appropriate antimicrobial agents, elevated MICs within the susceptible range may impact dosing strategy or antibiotic choice as well. Active surveillance of susceptibility and resistance trends is recommended for institutions to identify any changes that may necessitate adjustments in recommended antimicrobial therapy. OBJECTIVE: To describe the distribution of Pseudomonas aeruginosa and Streptococcus pneumoniae MICs from two Indianapolis hospitals. METHODS: This was a retrospective, descriptive study. A list of Pseudomonas aeruginosa and Streptococcus pneumoniae isolates from Wishard Health Services and Riley Hospital for Children in 2010-2011 was obtained. Only the first isolate was included from each subject per analysis period. Descriptive statistics were used for MIC data. A compilation of MIC distributions of antibiotics tested against the studied organisms at each institution was created. RESULTS: Streptococcus pneumoniae isolates had increasing MICs reported between 2010 and 2011 for amoxicillin, cefotaxime and vancomycin at Wishard Health Services. Additionally, Streptococcus 
pneumoniae had increasing MICs reported between 2010 and 2011 for penicillin and ceftriaxone at Riley Hospital for Children. Though the MIC values for Streptococcus pneumonia isolates for levofloxacin showed 100\% susceptibility in both 2010 and 2011, there were MIC values of $\geq 1 \mathrm{mg} / \mathrm{L}$ in only $77.8 \%$ of isolates in 2010 compared to $91.4 \%$ in 2011 at Riley Hospital. Pseudomonas aeruginosa isolates demonstrated increasing MICs between 2010 and 2011 for imipenem, meropenem and piperacillin/tazobactam at Riley Hospital and for cefepime, fluoroquinolones, carbapenems and aminoglycosides at Wishard Health Services. There was a larger percentage of Wishard Health Services Pseudomonas aeruginosa isolates with MICs above $16 \mathrm{mg} / \mathrm{L}$ for piperacillin/tazobactam in 2011 (26.44\%) versus 2010 (21.62\%). The MIC values for Pseudomonas aeruginosa isolates in regards to ciprofloxacin showed $>90 \%$ susceptibility in both 2010 and 2011, however in 2010, only 4\% had an MIC value of $1 \mathrm{mg} / \mathrm{L}$ compared to $8 \%$ in 2011 . CONCLUSION: Actual categorization of resistance did not significantly differ between 2010 and 2011, thus illustrating that resistance rates may not reflect potential shifts in MIC distribution. Increased MIC values suggest potential development of resistance. This data may be further utilized to optimize patient-specific antimicrobial choice and dosing recommendations for the treatment of specific bacteria at Riley Hospital for Children and Wishard Health Services.

\section{PEDIATRIC EDUCATION DIAGNOSIS SURVEY (PEDS): ANALYZING PEDIATRIC EDUCATION WITHIN PHARMD PROGRAMS IN THE UNITED STATES}

Phillip Weddle, Terri Warholak, and Hanna Phan University of Arizona College of Pharmacy, Tucson, AZ, USA pweddle@pharmacy.arizona.edu

BACKGROUND: Almost every pharmacist will encounter the pediatric population. However, historically, there has been a deficiency of pediatric education within Doctorate of Pharmacy (PharmD) programs in the United States. This lack of attention in a prominent area of practice has led to a sense of unease on the part of some pharmacists when dealing with pediatric patients. Developing an understanding of the current state of pediatric pharmacy education could spur curriculum changes within PharmD programs if deficiencies are identified. METHOD: This study involved a cross-sectional, descriptive survey. The questionnaire was sent via email to members of the American College of Clinical Pharmacy (ACCP) Pediatric Practice and Research Network (PRN). The questionnaire consisted of demographic information as well as questions concerning pediatric didactic and experiential education. The institution type and geographic location will be analyzed for differences in pediatric didactic and experiential education. Chi-square tests were used to compare rotation types and the percentage of students participating in pediatric Advance Pharmacy Practice Experience (APPE) rotations. Student t-tests and analysis of variance were performed to analyze the number of hours spent on pediatric education. RESULTS: Questionnaires were completed by faculty from 36 of 124 Schools or Colleges of Pharmacy (29\%) and included 26 dedicated pediatric faculty, 9 adjunct pediatric faculty, and 1 other (non-pediatric faculty) who was the primary instructor for pediatric education. Fifty-five percent of the PharmD programs were statefunded and $45 \%$ were private. The average reported number of pediatric didactic hours was $16.3 \pm 19.2$ hours and $23.8 \pm 24.6 \%$ of students participated in pediatric APPE rotations. Pediatric didactic education was similar between private and state funded institutions $(p=0.24)$ and geographic regions $(p=0.74)$. The percentages of students taking the different types of experiential rotations were similar between state funded and private institutions $(\mathrm{p}=0.64)$. There was a significant difference between regions for the percentage of students participating in pediatric APPE rotations $(\mathrm{p}<0.001)$. Specifically, the Northeast and Midwest regions showed a higher percentage than the rest of the United States $(\mathrm{p}<0.001)$. Programs commonly reported the following rotation experiences: general pediatrics $(78 \%)$, pediatric critical care $(69 \%)$, neonatal critical care $(67 \%)$, and pediatric ambulatory care $(28 \%)$. CONCLUSION: Pediatric didactic and experiential education appears to be consistent between state and privately-funded institutions as well as between geographic regions with the exception of a higher percentage of students in the Northeast and Midwest participating in pediatric APPE. Pediatric education seems to be lacking within PharmD programs given limited didactic hours and proportion of students in pediatric APPEs. Additional assessment of a larger sample of schools should be considered for future study. Further development of pediatric education in the PharmD curriculum, including APPE rotations, should be considered to help prepare PharmD students for general practice.

\section{EVALUATION OF VANCOMYCIN DOSING IN PEDIATRIC PATIENTS}

Maria Whitmore, ${ }^{1}$ Colleen Scherer, ${ }^{1}$ John Lock, ${ }^{2}$ and Elizabeth Moore ${ }^{2}$

${ }^{1}$ Peyton Manning Children's Hospital at St. Vincent Hospital, Indianapolis, Indiana, USA, ${ }^{2}$ St. Vincent Hospital, Indianapolis, Indiana, USA jmwhitmo@stvincent.org

INTRODUCTION: Traditional vancomycin dosing for infants $>1$ month of age and children has been 40-60 $\mathrm{mg} / \mathrm{kg} /$ day with a goal trough serum concentrations of $5-15 \mathrm{mg} / \mathrm{L}$. However, newer guidelines recommend higher trough serum vancomycin concentrations ( $>10$ $\mathrm{mg} / \mathrm{L}$ ) to treat serious gram positive infections. During 
a quality review we discovered that at the $45 \mathrm{mg} / \mathrm{kg} /$ day divided every 8 hours, our serum trough concentrations were consistently less than $10 \mathrm{mg} / \mathrm{L}$. Additionally, with dose adjustments, it was taking more than 48 hours to reach target serum concentrations. In order to achieve our target serum concentrations, in early 2009 we increased our empiric dosing to $60 \mathrm{mg} / \mathrm{kg} /$ day divided every 8 hours. The objective of our evaluation was to determine if our updated empiric dosing regimen resulted in trough concentrations of $10-15 \mathrm{mg} / \mathrm{L}$. METHODS: All infants and children seen at our institution between July 2010 and June 2012 who received vancomycin $60 \mathrm{mg} / \mathrm{kg} /$ day divided every 8 hours and had a trough vancomycin concentration measured prior to the third to sixth dose. Patients were excluded if their dosing was non-compliant with the updated institution empiric dosing and monitoring recommendations (less than $18 \mathrm{mg} / \mathrm{kg} /$ dose or greater than $22 \mathrm{mg}$ / $\mathrm{kg} /$ dose or frequency not every 8 hours), they received repeat courses of vancomycin, they were treated for meningitis with different dosing recommendations, or they received extracorporeal membrane oxygenation and/or renal replacement therapy. Data were collected from the electronic medical and pharmacokinetic monitoring records and included demographic data such as age, weight and gender along with infectious diagnosis. Laboratory values collected included initial blood urea nitrogen (BUN) and serum creatinine (Cr) within 24 hours of initiating vancomycin dosing. Vancomycin dose adjustments and corresponding repeat trough concentrations were also collected. RESULTS: Of the 173 eligible patients, the median trough serum vancomycin concentration was 9.3 $\mathrm{mcg} / \mathrm{mL}$ (range $1.3-61.4 \mathrm{mg} / \mathrm{L}$ ) and $23.1 \%$ had a vancomycin trough concentration within $10-15 \mathrm{mg} / \mathrm{L}$. Of the $39(22.5 \%)$ patients who had a vancomycin concentration above range, the majority $(74.4 \%)$ were treated in the PICU. Most of the patients with a high concentration treated in the PICU $(n=29)$ were $<1$ year of age $(65.5 \%)$. Vancomycin regimens were adjusted for $105(60.7 \%)$ patients. Of those adjusted, $23 \%$ were for dosing, $36 \%$ for frequency and $41 \%$ for both dosing and frequency. Overall, $42.9 \%(24 / 56)$ of patients with repeat vancomycin concentrations were within therapeutic range after their initial regimens were adjusted. CONCLUSION: Our increased empiric vancomycin dosing of $60 \mathrm{mg} / \mathrm{kg} /$ day divided every 8 hours was not able to attain our goal serum trough concentrations of $10-15 \mathrm{mg} / \mathrm{L}$ in the majority of our patients. Future studies are warranted to determine if increasing the frequency, and thereby the daily dosage, would result in higher trough concentrations.

\section{THE IMPACT OF A STANDARDIZED PROBIOTIC PROTOCOL IN A NEONATAL INTENSIVE CARE UNIT}

Heather Wild, Sherry Luedtke, Joyce Chuachingco, and Mubariz Naqvi
Texas Tech University Health Sciences Center School of Pharmacy, Amarillo, Texas, USA heather.wild@ttuhsc.edu

BACKGROUND: The use of probiotic supplementation in very low birth weight (VLBW) infants has been shown to improve feeding tolerance and reduce the incidence of necrotizing enterocolitis (NEC). While probiotic administration has been shown to be beneficial, controversy remains as to which probiotic supplement is most appropriate. Additionally, ideal dosage and duration of probiotic therapy is not well defined in this patient population. PURPOSE: The purpose of this study is to assess the impact of a standardized probiotic protocol in VLBW infants using the probiotic supplement ABC Dophilus (Bifidobacteria infantis, Streptococcus thermophilus, Bifidobacteria bifidus). METHODS: This observational study was conducted in the neonatal intensive care unit at Northwest Texas Hospital in Amarillo, Texas. All patients weighing less than 1500 grams at birth or born at less than 34 weeks gestation were eligible for study inclusion. All patients in the probiotic arm were compared to a retrospective control matched for gestational age and birth weight. The primary outcome evaluated was feeding tolerance, defined as the time to first full enteral feed (150 mL/kg/day). Secondary outcomes evaluated include incidence of NEC and blood culture results. RESULTS: 183 infants were enrolled in the study, 90 in the probiotic arm and 93 in the retrospective control arm. Main baseline characteristics did not differ significantly between the two groups. The average time to first full enteral feed was $10.4 \pm 6.7$ days in the control group and $11.4 \pm 7.5$ days in the probiotic group respectively $(\mathrm{p}=0.367)$. The incidence of NEC ( $\geq$ stage 2$)$ was higher in the control group when compared with the probiotic group ( 9 of 92 in the control group vs. 2 of 90 in the probiotic group, $p=0.058$ ). A total of 29 positive blood cultures were reported, none of which grew either Bifidobacterium or Streptococcus species. CONCLUSIONS: Routine probiotic supplementation with $A B C$ Dophilus did not appear to decrease the average time to first full enteral feed, but was potentially associated with a lower incidence of NEC.

\section{THE USE OF METOCLOPRAMIDE FOR THE TREATMENT OF NEONATAL REFLUX}

Mallary Wood ${ }^{2}$ Kay Kyllonen, ${ }^{1}$ and Barbara Kaplan ${ }^{1}$ ${ }^{1}$ The Cleveland Clinic, Cleveland, Ohio, USA, ${ }^{2}$ Nationwide Children's Hospital, Columbus, Ohio, USA mallary.wood@ nationwidechildrens.org

BACKGROUND: Gastroesophageal reflux (GER) is common in neonatal patients. One way to identify the clinical significance of GER is to document apnea, bradycardia and desaturation episodes; then, consider treatment based on the frequency of these events. Metoclopramide is amongst the treatment options; 
however, the 2009 Pediatric Gastroesophageal Reflux Clinical Practice Guidelines stated there is insufficient evidence to justify the routine use metoclopramide for the treatment of GER. This study evaluated the clinical utility of metoclopramide by investigating the change in frequency of apnea, bradycardia and desaturation episodes after initiation of therapy in neonatal patients. METHODOLOGY: An IRB-approved, chart review of 90 patients admitted to the neonatal intensive care unit at the Cleveland Clinic Main Campus receiving at least 72 hours of metoclopramide therapy was performed. The number of apnea, bradycardia and desaturation episodes 5 days before treatment was compared to the number of episodes during the 5 days after 72 hours of treatment. Exclusion criteria: less than 72 hours of therapy, immediate post-operative use, and confirmed bowel obstruction, gastrointestinal hemorrhage, necrotizing enterocolitis, or seizures. Data describing patient demographics, GER medications, number and type of episode, and enteral feeds were collected. An alpha of less than 0.05 was statistically significant. A Wilcoxon signed rank test and linear regression model were used to evaluate the data. RESULTS: There were 116 charts evaluated and 90 patients met inclusion criteria. The average gestational age was $30.5 \pm 4.5$ weeks and the average birth weight was $1605.7 \pm 879.6$ grams. The median number of total episodes during the 5 days prior to treatment was 8.5 (range $0-66$ ) episodes. About $6.7 \%$ of the patients received concomitant proton pump inhibitors, $80 \%$ received histamine receptor antagonists and 50\% received caffeine. Majority of patients $(83.3 \%)$ were receiving enteral feeds at initiation of therapy. The median number of total episodes during the 5 days after treatment was 7 (range 0-65) episodes $(\mathrm{p}=0.216)$. A subgroup analysis looked at patients with $<2$ episodes prior to treatment $(n=25)$ and found a significant increase in median number of total episodes after treatment $(p=0.008)$; as well as a significant increase in desaturation episodes specifically $(\mathrm{p}=0.012)$. For $\mathrm{pa}$ tients with $\geq 2$ episodes prior to treatment $(n=65)$, there was a significant reduction in median number of total episodes ( $p=0.022$ ) after 72 hours of therapy. In these patients, a linear regression model demonstrated the number of total episodes prior to initiation of therapy was the only significant characteristic associated with the primary outcome $(\mathrm{p}<0.05)$. Metoclopramide significantly reduced the number of total reflux episodes in patients experiencing at least 2 episodes prior to initiation of therapy. The use of a proton pump inhibitor, histamine receptor antagonist or caffeine was not associated with a reduction in reflux episodes.

\section{ASSESSMENT OF WASTE MANAGEMENT FOR BATCH PREPARED IV SYRINGES AT THE KEN- TUCKY CHILDREN'S HOSPITAL}

Joseph Wright, ${ }^{1}$ Robert Kuhn, ${ }^{1,2}$ and Brian Gardner ${ }^{1,2}$ ${ }^{1}$ University of Kentucky College of Pharmacy, Lexington, Kentucky, USA, ${ }^{2}$ Kentucky Children's Hospital, Lexington, Kentucky, USA jawrig12@gmail.com

INTRODUCTION: Waste reduction is an essential aspect of hospital and pharmacy management. In an attempt to reduce wasted medications, the Kentucky Children's Hospital's satellite pharmacy investigated how altering the time of batched medication preparation could affect the number of doses returned, and reduces overall waste. METHODS: Batch preparations of IV syringes and Oral unit-dose syringes were tracked. Daily recording of the number of overall doses dispensed to each unit of the hospital (i.e., NICU, PICU) was conducted. Unused medications were collected and stored, and the numbered doses of each drug returned from all hospital units were recorded as well. Twenty-one days worth of data were included. The IV batch time was then changed from 7:00 to 13:00, and a period of one month was given to allow the pharmacy and staff to acclimate to the new procedures. Another seventeen days were recorded in the same manner, and the results compared against one another. RESULTS: The initial phase of analysis showed an average of $19 \%$ of all batched doses was wasted. For the IV syringes, an average of $28.2 \%$ of doses was returned, totaling 27.6 IV doses each day. Upon changing the IV batch time, while leaving the Oral batch as it was, the overall returns of IV syringes decreased to $22.3 \%$ or 20.9 syringes per day. This is contrasted with the oral syringes serving as a control, which actually saw a slight increase in daily return rates from $13.78 \%$ to $14.56 \%$. CONCLUSION: Changing the print time to after daily team rounds led to a $6 \%$ decrease in IV medication waste. Unfortunately the pharmacy software does not allow multiple print times of IV syringes, as this is proven to optimize waste reduction. Although it is a seemingly small change, a rudimentary cost analysis for this six dose reduction yielded impressive benefits including an overall savings of approximately $\$ 13,500$. This represents nearly a 0.5 technician FTE. Further research is in progress to determine exact amount of time and money being saved through a reduction in pharmacist and technician workflow, as well as drug and supply costs. Future changes to current procedures plus better optimization of the batch time are being investigated to yield an even greater impact on pharmaceutical waste and costs management. 